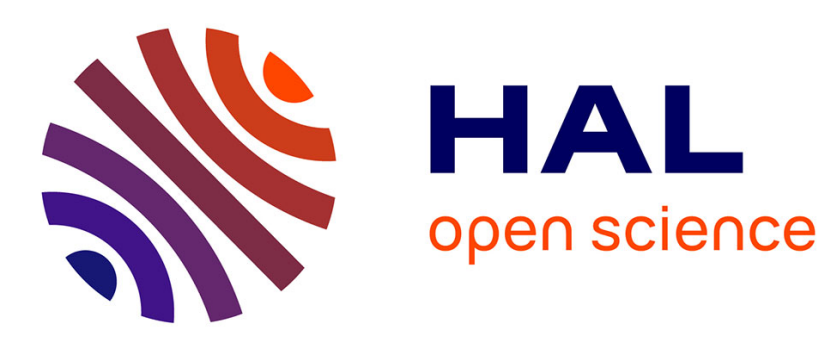

\title{
An accurate multi-regime SPH scheme for barotropic flows
}

\author{
A. Collé, J. Limido, J.-P. Vila
}

\section{To cite this version:}

A. Collé, J. Limido, J.-P. Vila. An accurate multi-regime SPH scheme for barotropic flows. Journal of Computational Physics, 2019, 388, pp.561 - 600. 10.1016/j.jcp.2019.03.028 . hal-03484796

\section{HAL Id: hal-03484796 \\ https://hal.science/hal-03484796}

Submitted on 20 Dec 2021

HAL is a multi-disciplinary open access archive for the deposit and dissemination of scientific research documents, whether they are published or not. The documents may come from teaching and research institutions in France or abroad, or from public or private research centers.
L'archive ouverte pluridisciplinaire HAL, est destinée au dépôt et à la diffusion de documents scientifiques de niveau recherche, publiés ou non, émanant des établissements d'enseignement et de recherche français ou étrangers, des laboratoires publics ou privés.

\section{다)(1) $(5$}

Distributed under a Creative Commons Attribution - NonCommercial| 4.0 International 


\title{
An Accurate Multi-Regime SPH Scheme for Barotropic Flows
}

\author{
A. Colléa,b, J. Limido ${ }^{a}$, J. P. Vila ${ }^{b}$ \\ ${ }^{\mathrm{a}}$ IMPETUS AFEA, 6 rue du Cers, 31330 Grenade sur Garonne, France \\ ${ }^{\mathrm{b}}$ Institut de Mathématiques de Toulouse, Institut national des sciences appliquées de Toulouse, 135 Avenue de \\ Rangueil, 31400 Toulouse, France
}

\begin{abstract}
This paper focuses on the use of a mesh-less numerical method to solve barotropic fluid flows: Smooth Particle Hydrodynamics (SPH). Such methods suffer from a severe lack of accuracy when evaluating state variables as the pressure field. SPH-ALE methods based on Riemann solvers significantly improve this evaluation but increase the scheme complexity and low-Mach issues are difficult to prevent. We propose an alternative scheme called $\gamma-S P H-A L E$ relying on the combination of the SPH-ALE formalism and a finite volume stabilizing low-Mach scheme. Applied to Monophasic Barotropic Euler Equations, its characteristics are detailed and evaluated through a nonlinear stability analysis highlighting CFL-like conditions on the scheme parameters. As far as we know, it is the first time such nonlinear analysis is performed on a SPH ALE scheme. Finally, its implementation on several academic test cases reveals that the proposed scheme actually increases both stability and accuracy, in reduced computation time, with respect to SPH ALE Riemann Solvers.
\end{abstract}

\section{Introduction}

Fluid dynamics systems involving violent flows or complex free surfaces are widely spread both in nature (breaking waves) and in technological applications (fragmentation, mixing). Being able to reproduce these situations is then a real asset to study, understand and solve the complex mechanisms involved. However, the numerical treatment of these configurations is particularly challenging. Finite element (FE) Eulerian solvers are classically preferred to manage such computations. Indeed, away from moving complex material interfaces, the Eulerian description of motion is well suited to describe the severe flow deformations induced by violent flows. Nevertheless, Eulerian solvers are subjected to a significant lack of accuracy when dealing with such interfaces. Some alternative Lagrangian approaches have emerged as the meshless methods and particularly the Smoothed Particle Hydrodynamics method (SPH). Since its emergence, it benefits from many researches aiming to improve its accuracy. As detailed in the next section, it is achieved thanks to stabilizing tools depending on the flow characteristics. We focus in this work on a new robust SPH scheme applied to the monophasic Barotropic Euler Equations. It benefits from the Arbitrary Lagrangian Eulerian (ALE) framework [1] and a stabilizing Low-Mach Scheme inherited from the Finite Volume (FV) context [2, 3].

\subsection{Smoothed Particle Hydrodynamics}

The SPH is a Lagrangian particle method used to solve partial differential equations (PDE). The computational domain is discretized in a set of interpolation points interpreted as particles interacting between themselves and carrying material properties. Originally developed by Lucy [4] for astrophysical problems and by Gingold \& Monaghan [5, 6, 7] for hydrodynamic applications (incompressible free surface flows), the method was then adapted by Benz $[8,9]$ to the solid mechanics by including material models.

Regarding fluid flows, Monaghan [6] proposed to approximate incompressible flows by artificially allowing a slight flow compressibility. He proposed then an explicit SPH formulation based on Weakly Compressible hypotheses (WCSPH). This approach relaxes the time step restriction and allows to treat complex free surface flows. As detailed by Lee et al. [10] and Fatehi et al. [11], such method bypasses the need to solve a Poisson equation to compute the pressure field (incompressible constraint, implicit resolution), and only relies on a simple equation of state (EOS). Restricted here to barotropic flows, as detailed in section 5.3, we use a linear 
EOS depending only on the flow density (the internal energy is not then a determinant variable for the flow behavior). The WCSPH formulation is particularly attractive for its conceptual simplicity, robustness, ability to handle large arbitrary deformations or to not produce diffusive interfaces and for its monolithic nature. However, for multi species cases, a particular treatment has to be done to handle the interface interactions (boundary terms) $[12,13,14]$. The proposed scheme detailed in section 4 is restricted to monophasic flows and disregards such interface thoughts. Despite being attractive, SPH schemes still suffer from well-known issues questioning their accuracy: lack of interpolation completeness, nonphysical oscillations, tensile instability, particle clumping. Regarding the completeness, some efforts have been done on the interpolation methods and particularly regarding the kernel functions $[15,16,17,18]$. Classical SPH interpolation is known to be not even $0^{\text {th }}$ order consistent [19]. To increase this order of consistency and improve the scheme accuracy, Johnson et al. [15] and Vila [16] proposed to use renormalized kernels (one order increase). To allow a consistency increase at any desired order, Dilts [17] worked with a Moving Least Square interpolation scheme and, more recently, Frontiere et al. [18] proposed a scheme based on Reproducing Kernels. To prevent nonphysical oscillations, two procedures are classically followed. The first, introduced by Colagrossi et al. [12], consists in periodically filtering the density field. It reduces the numerical noise by restoring the consistency between mass, density and volume. The second procedure is detailed by Marrone et al. [20]. They proposed a scheme called $\delta-S P H$ based on a density diffusive term aiming to smooth the spurious density oscillations. Other diffusive schemes are available in the literature $[21,22,23]$. In case of inviscid flows, these diffusive terms are generally combined with Monaghan's [5] artificial viscosity for stability reasons. Finally, regarding tensile instability and particle clumping, Monaghan proposed in [24] to add a corrective pressure term to the momentum equation. Based on the analysis of Swegle et al. [25], this term reduces the tensile instability by introducing a repulsive force which magnitude depends on the particle spacing. In order to prevent the formation of anisotropic particle distribution as particle clumping methods as XSPH [26, 7] or Particle Shifting Techniques (PST) [27, 28, 29, 30, 31, 32, 33] are also available in the literature and discussed in sections 1.2 and 4.3 .

Despite their stabilizing properties, these correction tools also raise conservation and consistency issues limiting their effectiveness [34]. In order to achieve robust and consistent stabilization, we focus in the next section on a different SPH framework introduced by Vila [1] and based on ALE considerations.

\subsection{Arbitrary Lagrangian Eulerian SPH}

The ALE formalism was firstly introduced for fluid mechanics modeling in a FE framework by Hirt [35, 36] and Noh [37]. The motivations were to combine the advantages of both Eulerian formulation (no motion of the frame of reference) and Lagrangian formulation (the frame of reference moves according to the material velocity). Indeed, regarding fluid flows, on one hand a Eulerian framework allows to handle severe deformations a Lagrangian cannot (due to high Lagrangian-grid distortions). On the other hand, when it comes to interface tracking (free surface flows), Lagrangian frameworks are more suited (as a fine Eulerian-grid is needed to accurately track the interface). In practice the combination relies on an arbitrary transport velocity field $v_{0}$ used to define the motion of the frame of reference. Similarly to Eulerian solvers, the flow evolution within the frame of reference is described thanks to flux terms but depending here on the relative ALE velocity $w=v-v_{0}$ (contrary to Eulerian flux terms depending directly on the material velocity $v$ ). Thus, as soon as $v_{0}$ is arbitrarily set (meaning not equal to zero for the Eulerian description, or to the flow velocity for the Lagrangian), the combination is achieved. Even if ALE methods allow to benefit from Eulerian and Lagrangian descriptions, they also experience some drawbacks of each sides. The major one being the very long computation times coming mainly from the algorithms and the meshing scales.

Vila [1] applied the SPH approximation technique in the ALE context and wrote the PDE based on a transport operator and flux vectors. It allows for a simple treatment of the arbitrary velocity field. In this case, as soon as $v_{0}$ is not set to the flow velocity, mass and momentum fluxes occur between particles and the interpolation point set is now a cloud of particles through which fluid flows. He took a particular interest in applying this method with Riemann Solvers (RS) building then a direct parallel with most of the Finite Volume (FV) techniques. The crucial aspect of the ALE formalism lies in the arbitrary property of the transport field $v_{0}$. Indeed, a smart choice of $v_{0}$ allows to increase the stability and robustness of the scheme without degrading its consistency. In [1] Vila suggested that, following Monaghan's XSPH method [26, 7], the ALE velocity field could be used to move the particles with a smooth velocity field. Recently, Lind et al. [38] and Fourtakas et al. [39] proposed an ALE SPH formulation in the incompressible context (ISPH). They work with a transport field proportional to the flow velocity (through a transition function) to increase accuracy thanks to the cohabitation of both Eulerian and Lagrangian areas. Oger et al. [30] also worked on SPH-ALE formulations using RS. Following the idea of Xu et al. [27], Lind et al. [28] and Adami et al.[29] regarding ISPH, he explored the stabilizing ability of a PST. Taking benefit from the ALE formalism, as suggested by Vila [1], 
Oger [30] implemented the PST through the ALE transport field $v_{0}$ (insuring the scheme consistency contrary to $[27,28,29,38,39])$. The motivation is to increase the accuracy by preventing the formation of anisotropic spatial particle distribution. Indeed, in a quasi-Lagrangian mode, particles can tend to organize themselves in anisotropic structures by mapping the fluid trajectories and generate significant interpolation errors. Similar PST were recently studied by Khayyer et al. [31] in ISPH, and by Sun et al. [32] in a classical SPH context to enhance the $\delta-S P H$ model. Zhang et al. [33] also extended a PST to deal with solid cases.

According to Oger's results [30], SPH-ALE Riemann Solvers (RSALE) formulations combined to PST seem convincing. Note that RS are attractive for their accuracy as they provide global solutions built on the combination of exact or approximate solutions to local Riemann problems. See Toro [40] for a detailed review. However, such accuracy level is achieved at the cost of an extreme solving complexity leading to excessive computation times. Many formulations are available in the literature but, later in this work, we use a MUSCL HLLC RS with a Sweby slope limiter as a reference. Solving a Riemann problem consists in evaluating, at a discontinuity, both left and right states thanks to a space interpolation scheme and next, to deduce the corresponding intermediate state. The HLLC version is an approximate RS and consists in not only evaluating this intermediate state but also in restoring the intermediate contact surface. To increase the accuracy, a MUSCL interpolation scheme is generally used to interpolate the left and right states at the second order in space. It is also combined to slope limiters aiming to prevent spurious oscillations in case of severe discontinuities. All this process must be solved for each local Riemann problems (meaning for each particle interactions in case of SPH) which generates the RS complexity. In addition RS suffer from classical limitations in low-Mach regimes and then particularly in case of Weakly Compressible (WC) flows [41, 42]. It is due to the presence of pressure fluctuations amplified by a factor $\frac{1}{M}$ (where $M$ is the Mach number) in the discrete scheme (amplified spurious oscillations when $\mathrm{M}$ goes to zero). See [42] for more details.

\subsection{Finite volume low-Mach scheme}

As introduced above, RS behave poorly in low-Mach regimes due to excessive numerical diffusion generated by spurious pressure oscillations [41, 2, 42]. A large amount of stabilizing methods are available in the literature but we choose to focus on the work of Grenier, Vila et al. [2] and Lavalle, Vila et al. [3].

In [2] a numerical scheme implemented in a FV two-phase flow framework is detailed, for solving fluid sloshing issues. The idea is to use a stabilizing velocity term $v_{\gamma}$ to correct the flow velocity in the continuity and momentum equations (the flow velocity $v$ is replaced by the corrected velocity $\left(v-v_{\gamma}\right)$ ). $v_{\gamma}$ is proportional to the pressure difference between two neighboring cells and depends on a parameter $\gamma$ to adjust. Thanks to a non-linear stability analysis, it is proved that a CFL-like condition on $\gamma$ ensures the scheme stability. Its implementation on several academic test cases shows that this new low-Mach scheme is robust and accurate whichever flow regime, but also less dissipative than classical RS in low-Mach regimes. Lavalle, Vila et al. [3] combined the low-Mach scheme with an ALE formulation in the case of thin liquid films shared by a gas flow. In this ALE framework, $v_{\gamma}$ is used to correct the relative ALE velocity ( $w$ is replaced by the corrected velocity $\left.\left(w-v_{\gamma}\right)\right)$. It means that even in Lagrangian description $\left(v_{0}=v\right)$, if $\gamma \neq 0$ the flux terms are activated and act as additional stabilizing terms. It is similar to stabilizing techniques based on diffusive terms previously introduced [22, 23, 21]. As in [2], a CFL-like condition on $\gamma$ insures the scheme stability. Couderc et al. [43] recently adapted the FV low-Mach scheme in the case of shallow water models to provide both non-linear and linear stability conditions on the regularizing parameters. Note that as achieved by Violeau et al. [44] such linear analysis also allows to maximize the time step restriction and to evaluate the effect of a variation on some key features of the scheme (kernel choice, SPH operators, integration scheme).

In the SPH context, Clayer et al. [45], proposed a semi-implicit SPH scheme called $\gamma-S P H$ similar to Grenier's [2] FV low-mach scheme. The stabilizing velocity $v_{\gamma}$ is now used to correct the flow velocity $v$ in the continuity and momentum equations (as in [2]), but also to correct the particles position. $v_{\gamma}$ is then also used as a regularizing tool like PST introduced in the previous section. According to Clayer's [45] and Lavalle's [3] satisfying results, it seems relevant to adapt the coupling between the FV low-Mach scheme and the ALE formalism to the SPH framework.

\subsection{Structure of the paper}

First, in section 2, similarly to Grenier et al. [2], we introduce in the case of monophasic Barotropic Euler Equations a continuous scheme modified by the implementation of the low-Mach scheme. Its stability is demonstrated by exhibiting the stabilizing effects of the Low-Mach scheme building the basis of the present work. In section 3, we present the SPH-ALE discretization method (introduced by Vila in [1]) used to approximate the continuous modified scheme. The SPH approximation features are detailed as well as how they are applied to approximate a conservation law. The particular case of a law in its ALE formulation is exposed. In section 4, 
the discrete scheme $\gamma-S P H-A L E$ is proposed as a result of the SPH-ALE discretization of the continuous modified scheme (introduced in section 2). Its constitutive equations and their specific features are described. In section 5, a nonlinear stability analysis is performed on the proposed discrete scheme. Stability conditions are exhibited ensuring its conservativity, robustness, stability and consistency in a barotropic framework. To the authors knowledge, it is the first time such nonlinear analysis is completed on a SPH-ALE scheme. Finally, in section 6 , the scheme performances are evaluated on several academic test cases: isentropic shock tube, rotating square patch of fluid, oscillating drop and dam break.

\section{Continuous Framework}

As proposed by Grenier et al. [2], we introduce an implementation of the FV low-Mach scheme in a continuous framework. The corresponding modified monophasic Euler Equations are exhibited and the dissipative behavior insuring its stability is highlighted.

\subsection{Proposed Continuous Equations}

We recall the classical monophasic Euler equations

$$
\left\{\begin{array}{l}
\frac{\partial \rho}{\partial t}+\operatorname{div}(\rho \boldsymbol{v})=0 \\
\frac{\partial \rho \boldsymbol{v}}{\partial t}+\operatorname{div}(\rho \boldsymbol{v} \otimes \boldsymbol{v})+\nabla p=0
\end{array}\right.
$$

giving the following total energy conservation law

$$
\frac{\partial E}{\partial t}+\operatorname{div}((E+p) \boldsymbol{v})=0
$$

where we define the total energy $E$ as $E=\frac{1}{2} \rho\|v\|^{2}+\varphi \cdot \varphi=\rho \int_{\rho_{0}}^{\rho} \frac{p(r)}{r^{2}} d r$ is the total free energy and corresponds to the barotropic pressure. Note that according to the free energy definition it is straightforward that $\rho \frac{\partial \varphi}{\partial \rho}=\varphi+p$.

To take into account the FV Low-Mach scheme, we work with the modified velocity $\boldsymbol{w}=\boldsymbol{v}-\boldsymbol{\Gamma}$ where $\boldsymbol{\Gamma}$ is the Low-Mach stabilizing velocity term noted $v_{\gamma}$ in the previous section and defined here as

$$
\boldsymbol{\Gamma}=\frac{\gamma h}{\rho c_{0}} \nabla p
$$

$\rho, c_{0}$ and $p$ are respectively the density, sound speed and pressure. $\gamma$ is the dimensionless Low-Mach parameter and $h$ a small parameter representative of the mesh size. As introduced in section 1.1, in case of inviscid flows an artificial viscosity term [5] is implemented to improve the scheme stability. We set $\boldsymbol{\pi}$ this artificial viscosity and define it here as

$$
\boldsymbol{\pi}=\alpha \rho c_{0} h \boldsymbol{\nabla} \boldsymbol{v}
$$

where $\boldsymbol{v}$ is the material velocity and $\alpha$ the artificial viscosity parameter. (4)

Equations (1) can then be rewritten as the following modified system by taking into account $\boldsymbol{\Gamma}(3)$ and $\boldsymbol{\pi}$

$$
\left\{\begin{array}{l}
(i) \frac{\partial \rho}{\partial t}+\operatorname{div}(\rho \boldsymbol{w})=0 \\
(i i) \frac{\partial \rho \boldsymbol{v}}{\partial t}+\operatorname{div}(\rho \boldsymbol{v} \otimes \boldsymbol{w})+\operatorname{div}(p \boldsymbol{I} \boldsymbol{d}-\boldsymbol{\pi})=0
\end{array}\right.
$$

As in the classical case (2), equations (5) gives the following modified total energy equation

$$
\frac{\partial E}{\partial t}+\operatorname{div}\left([E+p] \boldsymbol{w}-\alpha \rho c_{0} h \boldsymbol{\nabla} \boldsymbol{v} \cdot \boldsymbol{v}\right)=-\frac{\gamma h}{\rho c_{0}}\|\nabla p\|^{2}-\alpha \rho c_{0} h \boldsymbol{\nabla} \boldsymbol{v}: \boldsymbol{\nabla} \boldsymbol{v} \leq 0
$$

We can exhibit the following source terms

$$
\begin{gathered}
S_{\gamma}=-\frac{\gamma h}{\rho c_{0}}\|\nabla p\|^{2} \\
S_{\alpha}=-\alpha \rho c_{0} h \nabla \boldsymbol{v}: \nabla \boldsymbol{v}
\end{gathered}
$$

Considering the modified equations $(5,6)$, we can see that the classical system $(1,2)$ is recovered when $h$ goes to zero. Besides, we can also see that both Low-Mach scheme (3) and Artificial viscosity (4) generate negative source terms $S_{\gamma}(7)$ and $S_{\alpha}(8)$ in the modified total energy equation (6). Leading to a total energy dissipation, it demonstrates their stabilizing effect. 


\subsection{Modified Total Energy Proof}

Multiplying $(5-i)$ by $\frac{\partial \varphi}{\partial \rho}$ gives the following free energy conservation law

$$
\frac{\partial \varphi}{\partial t}+\operatorname{div}(\varphi \boldsymbol{w})+\operatorname{pdiv}(\boldsymbol{w})=0
$$

Considering $(5-i i)$ and $(5-i)$, we get by multiplying by $\boldsymbol{v}$ the following kinetic energy conservation law

$$
\frac{\partial}{\partial t}\left[\rho \frac{\|\boldsymbol{v}\|^{2}}{2}\right]+\operatorname{div}\left(\rho \frac{\|\boldsymbol{v}\|^{2}}{2} \boldsymbol{w}\right)+\boldsymbol{w} \cdot \boldsymbol{\nabla} p-\operatorname{div}(\boldsymbol{\pi} \cdot \boldsymbol{v})=-\boldsymbol{\Gamma} \cdot \boldsymbol{\nabla} p-\boldsymbol{\pi}: \boldsymbol{\nabla} \boldsymbol{v}
$$

Finally, by gathering (9) and (10) we get

$$
\frac{\partial}{\partial t}\left[\rho \frac{\|\boldsymbol{v}\|^{2}}{2}+\varphi\right]+\operatorname{div}\left(\left[\rho \frac{\|\boldsymbol{v}\|^{2}}{2}+\varphi\right] \boldsymbol{w}\right)+\boldsymbol{w} \cdot \nabla p+\operatorname{pdiv}(\boldsymbol{w})-\operatorname{div}(\boldsymbol{\pi} \cdot \boldsymbol{v})=-\boldsymbol{\Gamma} \cdot \boldsymbol{\nabla} p-\boldsymbol{\pi}: \boldsymbol{\nabla} \boldsymbol{v}
$$

which is to the expected modified total energy equation (6).

We have formally demonstrated in this section that the implementation of the FV Low-Mach scheme combined to an artificial viscosity insures a dissipative behavior at a continuous level. However, if we want now to discretize these modified equations in space and time, such dissipative behavior cannot be directly insured. The aim of the present work is first to propose an approximation of the modified equations $(5,6)$ thanks to the SPH-ALE discretization. And second to provide, thanks to a nonlinear stability analysis, stability conditions on parameters $\alpha$ and $\gamma$ insuring the stability of the proposed discrete scheme.

\section{SPH-ALE Discretization}

Note that in the following $P$ defines the whole particle domain among which $i$ describes one particle. We set $V_{i}$ its neighboring particle set and we take $j \in V_{i}$. Finally $n \in \mathbb{N}$ defines the time step number and $d \in \mathbb{N}^{*}$ the space dimension. In this section we refer to $[1,19]$ for more details on SPH and SPH-ALE.

\subsection{Particle Approximation}

The SPH method relies on approximation features allowing to discretize the conservation laws thanks to a set of particles $\left(x_{i}(t), \omega_{i}(t)\right)_{i \in P}$ (respectively the particle position and weight). These particles move at a velocity $v_{i}=v\left(x_{i}, t\right)$ such that $v_{i}=\frac{d x_{i}}{d t}$ and their weights evolve under the effect of $v$ as

$$
\frac{d \omega_{i}}{d t}=\omega_{i} \operatorname{div}\left(v_{i}\right)
$$

Introducing a regularizing kernel $W(x, h)$ (where $h$ is the smoothing length and corresponds to the radius of its compact support), we can define the smoothed particle approximation of a function $\mathrm{f}(i)$ and of its derivative (ii)

$$
\left\{\begin{array}{c}
(i) \quad \Pi^{h}(f)_{i}=\sum_{j \in P} \omega_{j}(t) f_{j}(t) W_{i j}(t) \\
(i i) \quad \nabla \Pi^{h}(f)_{i}=\sum_{j \in P} \omega_{j}(t) f_{j}(t) \nabla W_{i j}(t)
\end{array}\right.
$$

where $W_{i j}(t)=W\left(x_{i}(t)-x_{j}(t), h\right)$ and $\nabla W_{i j}(t)=\operatorname{grad}_{x} W\left(x_{i}(t)-x_{j}(t), h\right)$. As introduced above, classical SPH suffers from a lack of interpolation completeness which can be compensated by using renormalized kernels. Vila actually proposed to replace $\boldsymbol{\nabla} \boldsymbol{W}_{i j}$ by an alternative gradient $\boldsymbol{A}_{i j}$ defined by $\boldsymbol{A}_{i j}=B_{i j} \nabla \mathbf{W}_{\mathbf{i j}}$ where $B_{i j}=\frac{1}{2}\left(B_{i}+B_{j}\right)$ and $B$ is the renormalization matrix introduced by Johnson et al. [15] and Vila [16]. It is defined as

$$
B_{i}=\left(E_{i}\right)^{-1} \text { with } \forall(\alpha, \beta) \in\{1, \ldots, d\}^{2}, E_{i}^{\alpha \beta}=\sum_{j} \omega_{j}\left(x_{j}^{\beta}-x_{i}^{\beta}\right) \nabla W_{i j}^{\alpha}
$$

We set in the following content $\mathbf{n}_{\mathbf{i j}}=\frac{\mathbf{A}_{\mathbf{i j}}}{\left\|\mathbf{A}_{\mathbf{i j}}\right\|}$.

Regarding the derivative definition $(i i)$, we can actually define two additional derivative operators as

$$
\begin{aligned}
D_{h} f_{i} & =\boldsymbol{\nabla}\left(\Pi^{h} f\right)_{i}-f_{i} \boldsymbol{\nabla}\left(\Pi^{h} 1\right)_{i} \\
& =\sum_{j \in P} \omega_{j}(t)\left(f_{j}(t)-f_{i}(t)\right) \boldsymbol{A}_{i j}(t)
\end{aligned}
$$




$$
\begin{aligned}
D_{h}^{*} f_{i} & =\boldsymbol{\nabla}\left(\Pi^{h} f\right)_{i}+f_{i} \boldsymbol{\nabla}\left(\Pi^{h} 1\right)_{i} \\
& =\sum_{j \in P} \omega_{j}(t)\left(f_{j}(t)+f_{i}(t)\right) \boldsymbol{A}_{i j}(t)
\end{aligned}
$$

Note that if we consider symmetric kernels such that $W(x, h)=W(-x, h)$ and $\boldsymbol{\nabla} W_{i j}=-\nabla W_{j i}$, choosing $B_{i j}=\frac{1}{2}\left(B_{i}+B_{j}\right)$ actually keeps the anti-symmetric property of the kernel gradient (meaning that $\mathbf{A}_{\mathbf{i j}}=-\mathbf{A}_{\mathbf{j i}}$ ). It ensures that, $-D_{h}^{*}$ corresponds to the adjoin operator of $D_{h}$ in the sense of the following discrete scalar product

$$
(., .)_{h}:(\varphi, \psi) \rightarrow \sum_{i \in P} \omega_{i} \varphi_{i} \psi_{i}
$$

approximating the scalar product in $\mathcal{L}^{2}\left(\mathbb{R}^{d}\right)$.

Besides, it is proved [1] that $D_{h}$ strongly approximates the gradient operator $\nabla$ in a sense that for a regular function $\varphi$

$$
\sup _{i \in P}\left\|D_{h} \varphi_{i}-\nabla \varphi_{i}\right\|=0 \text { as } h \text { and } \Delta x \rightarrow 0
$$

Such convergence property is achieved thanks to the following approximation results

$$
\left\{\begin{array}{l}
\exists K_{i, 1}^{n} \in \mathbb{R}^{+, *} /\left\|\sum_{j} \boldsymbol{A}_{i j} w_{j}^{n}\right\| \leq K_{i, 1}^{n} \text { with also } K_{1}^{n}=\max _{i} K_{i, 1}^{n} \text { and } K_{1}=\max _{n} K_{1}^{n} \\
\exists C_{i}^{n} \in \mathbb{R}^{+, *} / \sum_{j} w_{j}^{n}\left\|\boldsymbol{A}_{i j}\right\| \leq \frac{C_{i}^{n}}{h} \text { with also } C^{n}=\max _{i} C_{i}^{n} \text { and } C=\max _{n} C^{n}
\end{array}\right.
$$

Note that these constants stay bounded both in regular or arbitrary particle distributions. See Section 6.3 fig. 7 and [19] for an analysis in the regular case. According to the derivative form (14), we can show that $\sum_{i \in P} \omega_{i} D_{h}^{*} f_{i}=0$ (thanks to the anti-symmetric property of $\boldsymbol{A}_{i j}$ ).

The idea is now to use these tools and their property to propose an approximation of a conservation law.

\subsection{Conservation Laws}

We consider laws in the following conservation form

$$
L_{v}(\Phi)+\operatorname{div} F(x, t, \Phi)=S(x, t, \Phi), t \in \mathbb{R}^{+}, x \in \mathbb{R}^{d}
$$

where $v \in \mathbb{R}^{d}$ is a regular vector field, $\Phi \in \mathbb{R}^{p}$ is the vector of the conserved variables, $F=\left(F^{\alpha}\right)_{\alpha}^{d}$ is the flux vector corresponding to the conservation law composed of $d$ vectors $F^{\alpha} \in \mathbb{R}^{p}, S=\left(S^{l}\right)_{l}^{p}$ is the source term in $\mathbb{R}^{p}$ and $L_{v}: \Phi \rightarrow \frac{\partial \Phi}{\partial t}+\operatorname{div}(\boldsymbol{\Phi} \otimes \boldsymbol{v})$ is the transport operator associated to $v$.

To approximate the law (17) considering the moving particle set $\left(x_{i}(t), \omega_{i}(t)\right)_{i \in P}$, we firstly introduce the weak formulation of (17) defined by $\forall \varphi \in \mathcal{C}_{0}^{p}\left(\mathbb{R}^{d} \times \mathbb{R}^{+, *}\right)$,

$$
\int_{\mathbb{R}^{d} \mathbb{R}^{+}}\left[\Phi \cdot L_{v}^{*}(\varphi)+F(x, t, \Phi) \cdot \nabla \varphi+S \cdot \varphi\right] d x d t=0
$$

where $-L_{v}^{*}$ is the dual operator of $L_{v}$ defined as $L_{v}^{*}: \varphi \rightarrow \frac{\partial \varphi}{\partial t}+\boldsymbol{v} \cdot \operatorname{grad}(\varphi)$.

Considering the discrete scalar product (15) we are able to discretize the weak formulation (18) in space as $\forall \varphi \in \mathcal{C}_{0}^{p}\left(\mathbb{R}^{d} \times \mathbb{R}^{+, *}\right)$,

$$
\int_{\mathbb{R}^{+}}\left[\left(\Phi, L_{v}^{*}(\varphi)\right)_{h}+\sum_{\alpha=1, \ldots, d}\left(F^{\alpha}(\Phi), \nabla^{h} \varphi\right)_{h}+\left(S+R_{h}(\Phi), \varphi\right)_{h}\right] d t=0
$$

where $R_{h}(\Phi)$ corresponds to the residual of the equation (as the artificial viscosity) and $\nabla^{h}$ to a derivative operator approximating $\nabla$.

At this stage two crucial property must be enforced by the discrete scheme (19)

- the weak consistency with (17)

- the conservation in the sense that $\frac{d}{d t}\left(\int_{\mathbb{R}^{d}} \Phi d x\right)=\int_{\mathbb{R}^{d}} S d x$ 
Consistency As regards this first aspect a Lax-Wendroff like theorem actually provides sufficient conditions to ensure a good limit weak solution:

\section{Theorem2.2.1}

Let $\bar{\Phi}^{\triangle}=\sum_{j \in P} \Phi_{j}(t) \chi_{B_{j}}(x)$ the function associated with the sequence $\left(\Phi_{j}(t)\right)_{j \in P}$ of regular functions of $\mathrm{t}$ defined by the scheme $(19) . \chi_{B_{j}}(x)$ is the characteristic function of the set $B_{i}(t)$ (see [1] for more details). We suppose that:

1. the function $\bar{\Phi}^{\triangle}$ converges boundedly almost everywhere to $\Phi$ when $\mathrm{h}$ and $\Delta x$ go to zero,

2. $\forall \varphi \in\left[\mathcal{C}_{0}\left(\mathbb{R}^{d} \mathrm{x} \mathbb{R}^{+, *}\right)\right]^{p} \sup _{i \in P}\left\|\nabla^{h} \varphi_{i}-\nabla \varphi_{i}\right\| \rightarrow 0$ as $\mathrm{h}$ and $\Delta x \rightarrow 0$,

3. $\lim _{h}$ and $\Delta x \rightarrow 0, \int_{\mathbb{R}^{+}}\left(R_{h}\left(\bar{\Phi}^{\triangle}\right), \varphi\right)_{h} d t=0$,

Then $\Phi$ is a weak solution of the model PDE (17) in the sense of Definition (18).

Thus, regarding the previous section by choosing $\nabla^{h}=D_{h}$ we just have to enforce theorem 2.2 .1 condition 3 to achieve the expected weak consistency of the discrete scheme (19). Note that the theorem convergence condition 2 actually means that

$$
\int_{\mathbb{R}^{+}}\left[\sum_{\alpha=1, \ldots, d}\left(F^{\alpha}(\Phi), \nabla^{h} \varphi\right)_{h}\right] d t \rightarrow \int_{\mathbb{R}^{d} \mathbf{x}^{+}}[F(x, t, \Phi) . \boldsymbol{\nabla} \varphi] d x d t, \text { as } h \text { and } \Delta x \text { go to zero. }
$$

In standard SPH, to achieved such convergence property we need that the ratio $\frac{\Delta x}{h}$ (where $\mathrm{h}$ is the smoothing length and $\Delta x$ is the particle spacing) goes to zero or to choose particular values (classically $h=1.2 \Delta x$, see [19]). But using the renormalization (introduced in section 3.1) allows to relax this condition and to just take $\frac{\Delta x}{h}=O(1)($ see $[1])$.

Conservation For this second aspect, we need to firstly detail the discrete scheme (19). Indeed, having $\nabla^{h}=D_{h}$ we can actually write by duality $\forall \varphi \in \mathcal{C}_{0}^{p}\left(\mathbb{R}^{d} \mathrm{x}^{+, *}\right)$,

$$
\int_{\mathbb{R}^{+}}\left(L_{v}(\Phi)_{i}+\sum_{\alpha=1, \ldots, d} D_{h}^{\alpha, *}\left(F^{\alpha}\right)_{i}-\left(S_{i}+R_{h}(\Phi)_{i}\right), \varphi\right)_{h} d t=0
$$

corresponding to

$$
\frac{d}{d t}\left(\omega_{i} \Phi_{i}\right)+\omega_{i} \sum_{\alpha=1, \ldots, d} D_{h}^{\alpha, *}\left(F^{\alpha}\right)_{i}=\omega_{i}\left(S_{i}+R_{h}(\Phi)_{i}\right)
$$

Thus, disregarding the residuals, we have on the whole particle domain

$$
\frac{d}{d t}\left(\sum_{i \in P} \omega_{i} \Phi_{i}\right)=\sum_{i \in P} \omega_{i} S_{i}
$$

which is a discrete version of the conservation condition $\frac{d}{d t}\left(\int_{\mathbb{R}^{d}} \Phi d x\right)=\int_{\mathbb{R}^{d}} S d x$.

Having these results in the case of a general Lagrangian conservation law (17) we present now its ALE version.

\subsection{ALE Formulation}

We consider now a regular transport field $\boldsymbol{v}_{0}(x, t)$ ruling the particle motion and defining the motion of the frame of reference. We can rewrite the conservation law (17) using $\boldsymbol{v}_{0}$ as transport field

$$
L_{v_{0}}(\Phi)+\operatorname{div}\left(F_{E}(\Phi)-\Phi \bigotimes v_{0}\right)=S(\Phi)
$$

where $F_{E}$ is the Eulerian flux vector (meaning the flux vector when $v_{0}=0$ ). Considering now the modified equations (5), we can write its ALE formulation (22) with

$$
F_{E}=\left(\begin{array}{ccc}
\rho\left(v_{x}-v_{\gamma x}\right) & \rho\left(v_{y}-v_{\gamma y}\right) & \rho\left(v_{z x}-v_{\gamma z}\right) \\
-\rho v_{x}\left(v_{x}-v_{\gamma x}\right)+p-\pi_{x x} & -\rho v_{x}\left(v_{y}-v_{\gamma y}\right)-\pi_{x y} & -\rho v_{x}\left(v_{z x}-v_{\gamma z}\right)-\pi_{x z} \\
-\rho v_{y}\left(v_{x}-v_{\gamma x}\right)-\pi_{y x} & -\rho v_{y}\left(v_{y}-v_{\gamma y}\right)+p-\pi_{y y} & -\rho v_{y}\left(v_{z x}-v_{\gamma z}\right)-\pi_{y z} \\
-\rho v_{z}\left(v_{x}-v_{\gamma x}\right)-\pi_{z x} & -\rho v_{z}\left(v_{y}-v_{\gamma y}\right)-\pi_{z y} & -\rho v_{z}\left(v_{z x}-v_{\gamma z}\right)+p-\pi_{z z}
\end{array}\right)
$$


Setting $\boldsymbol{w}=\boldsymbol{v}-\boldsymbol{v}_{0}-\boldsymbol{v}_{\gamma}$ we can write $(22)$ as

$$
L_{v_{0}}(\Phi)+\operatorname{div}\left(F_{A L E}(\Phi)\right)=0
$$

where

$$
F_{A L E}(\Phi)=\left(\begin{array}{ccc}
\rho w_{x} & \rho w_{y} & \rho w_{z} \\
\rho v_{x} w_{x}+p-\pi_{x x} & \rho v_{x} w_{y}-\pi_{x y} & \rho v_{x} w_{z}-\pi_{x z} \\
\rho v_{y} w_{x}-\pi_{y x} & \rho v_{y} w_{y}+p-\pi_{y y} & \rho v_{y} w_{z}-\pi_{y z} \\
\rho v_{z} w_{x}-\pi_{z x} & \rho v_{z} w_{y}-\pi_{z y} & \rho v_{z} w_{z}+p-\pi_{z z}
\end{array}\right)
$$

Also read as

$$
L_{v_{0}}(\Phi)+\left(\begin{array}{c}
\operatorname{div}(\rho \boldsymbol{w}) \\
\operatorname{div}(\rho \boldsymbol{v} \otimes \boldsymbol{w}+p \boldsymbol{I} \boldsymbol{d}-\boldsymbol{\pi})
\end{array}\right)=0
$$

Following the process exposed in the previous section, we can discretize equation (23) on a set of moving particles $\left(x_{i}(t), \omega_{i}(t)\right)_{i \in P}$ resulting in the following equation set

$$
\left\{\begin{array}{l}
\frac{d \boldsymbol{x}_{i}}{d t}=\boldsymbol{v}_{0 i} \\
\frac{d \omega_{i}}{d t}=\omega_{i} d i v_{h}\left(\boldsymbol{v}_{0}\right) \\
\frac{d}{d t}\left(\omega_{i} \Phi_{i}\right)+\omega_{i} \nabla_{h}^{*}\left(F_{A L E}(\Phi)\right)=0
\end{array}\right.
$$

where $\boldsymbol{v}_{0 i}=\boldsymbol{v}_{0}\left(\boldsymbol{x}_{i}, t\right)$ and $d i v_{h}$ a discrete approximation of the divergence operator. According to the previous subsection, the idea is to choose $\nabla^{h}$ such that the LHS term of equation (20) can be recovered by generating conservative and consistent residuals.

\section{Proposed Discrete Scheme $\gamma-S P H-A L E$}

Based on Section 2 and 3 results we introduce now the proposed $\gamma-S P H-A L E$ scheme coupling the FV lowMach scheme [2, 3] and the SPH-ALE formulation [1] applied to the monophasic Barotropic Euler Equations. It corresponds to a discrete version of the continuous modified scheme $(5,6)$ introduced in section 2.

\subsection{Fundamental Discrete Equations}

Several choices are admissible for the derivative operator $\nabla^{h}$ in equation (25) and we propose to approximate the continuous modified scheme $(5,6)$ with the following scheme discretized in space

$$
\left\{\begin{array}{l}
\frac{d \boldsymbol{x}_{i}}{d t}=\boldsymbol{v}_{0 i} \\
\frac{d \omega_{i}}{d t}=\omega_{i} \sum_{j} \omega_{j}\left\langle\left(\boldsymbol{v}_{0 j}-\boldsymbol{v}_{0 i}\right), \boldsymbol{A}_{i j}\right\rangle \\
\frac{d \omega_{i} \rho_{i}}{d t}=-\omega_{i} \sum_{j} \omega_{j}\left(\rho_{j}+\rho_{i}\right)\left\langle\boldsymbol{w}_{i j}, \boldsymbol{A}_{i j}\right\rangle \\
\frac{d \omega_{i} \rho_{i} \boldsymbol{v}_{i}}{d t}=-\omega_{i} \sum_{j} \omega_{j}\left(\rho_{j} \boldsymbol{v}_{j}+\rho_{i} \boldsymbol{v}_{i}\right)\left\langle\boldsymbol{w}_{i j}, \boldsymbol{A}_{i j}\right\rangle-\omega_{i} \sum_{j} \omega_{j}\left(p_{i j} \boldsymbol{A}_{i j}-\boldsymbol{\pi}_{i j}\right)
\end{array}\right.
$$

With $\boldsymbol{w}_{i j}=\frac{1}{2}\left(\boldsymbol{w}_{i}+\boldsymbol{w}_{j}\right)-\boldsymbol{\Gamma}_{i j}$.

Variables $x, \omega, \rho, v$ and $p$ refer respectively to the position, volume, density, velocity and pressure of the particles. $\boldsymbol{w}$ corresponds to the relative ALE velocity defined by $\boldsymbol{w}=\boldsymbol{v}-\boldsymbol{v}_{0}$. We recall that as detailed in section $1.2, \boldsymbol{v}_{0}$ is the arbitrary transport velocity field defining the description of motion (Lagrangian, Eulerian, PST see section 4.3). We also define $p_{i j}=p_{i}+p_{j}, \boldsymbol{\Gamma}_{i j}$ corresponds to the stabilizing term coming from the FV low-Mach scheme (30) and $\boldsymbol{\pi}_{i j}$ corresponds to the artificial viscosity (28). Note that $\langle.,$.$\rangle defines the usual$ scalar product on $\mathbb{R}^{d}$.

The integration is performed thanks to a second order Runge-Kutta Heun (RK2H) time integration scheme defined by

$$
\left\{\begin{array}{l}
\bar{\Theta}^{n+1}=\Theta^{n}+\Delta t \frac{d}{d t}\left(\Theta^{n}\right) \\
\Theta^{n+1}=\frac{1}{2}\left(\Theta^{n}+\bar{\Theta}^{n+1}+\Delta t \frac{d}{d t}\left(\bar{\Theta}^{n+1}\right)\right)
\end{array} \quad \text { with } \Theta=\left(\begin{array}{c}
\boldsymbol{x} \\
\omega \\
\omega \rho \\
\omega \rho \boldsymbol{v}
\end{array}\right)\right.
$$

Note that we choose RK2H for, as detailed by Shu and Osher [46], being a combination of two first order steps, it preserves the stability achieved in section 5 (formally proved in Appendix B). 


\subsection{Dissipative Features}

\subsubsection{Artificial viscosity}

To improve the stability of the scheme Monaghan's artificial viscosity is usually introduced in the momentum equation pressure term [5]. As introduced in section 2.1, we propose to approximate the continuous term (4) as

$$
\boldsymbol{\pi}_{i j}=\alpha_{i j}\left(\boldsymbol{v}_{j}-\boldsymbol{v}_{i}\right)
$$

Similarly to (4) we set

$$
\alpha_{i j}=\alpha \bar{c}_{i j} \bar{\rho}_{i j}\left\|\boldsymbol{A}_{i j}\right\| \quad, \alpha \geq 0
$$

with $\bar{c}_{i j}=\frac{1}{2}\left(c_{i}+c_{j}\right)$ and $\bar{\rho}_{i j}=\frac{1}{2}\left(\rho_{i}+\rho_{j}\right)$. It depends on a parameter $\alpha$ (dimensionless) which value is calibrated thanks to a nonlinear stability analysis (see Section 5). Seen as a diffusive term, the idea is to adjust this parameter in order to minimize the amount of artificial diffusion induced in the scheme.

\subsubsection{Low-Mach Scheme}

The coupling between the SPH version of the FV Low-Mach scheme [2, 3, 45] and the SPH-ALE formalism [1] is achieved by the term $\boldsymbol{\Gamma}_{i j}$ through the mean relative ALE velocity $\boldsymbol{w}_{i j}$. Defined as

$$
\boldsymbol{\Gamma}_{i j}=\gamma_{i j}\left(p_{j}-p_{i}\right) \boldsymbol{n}_{i j}
$$

with

$$
\gamma_{i j}=\frac{\gamma}{2 \tilde{c}_{i j} \tilde{\rho}_{i j}} \quad, \gamma \geq 0
$$

It corresponds to a discrete version of the continuous term (3) introduced in section 2.1. It depends on the parameter $\gamma$ (dimensionless) and, similarly to the artificial viscosity, its value is calibrated thanks to a nonlinear stability analysis (Section 5).

We actually set $\gamma_{i j}=\gamma \frac{\widetilde{\kappa}_{i j}^{\prime}}{2 \tilde{c}_{i j}^{3}}$ where $\kappa^{\prime}$ corresponds to the second derivative of the free energy $\varphi$. Taylor's expansion of $\varphi$ gives a certain $\theta_{i j}$ in $[0,1]$ such that $\tilde{\kappa}_{i j}^{\prime}=\kappa^{\prime}\left(\left(1-\theta_{i j}\right) \rho_{i}+\theta_{i j} \rho_{j}\right)=\kappa^{\prime}\left(\tilde{\rho}_{i j}\right)$. And we define $\tilde{c}_{i j}$ such that $\widetilde{\kappa}_{i j}^{\prime}=\frac{\tilde{c}_{i j}^{2}}{\tilde{\rho}_{i j}}$.

Note that here $\boldsymbol{\Gamma}_{i j}$ corresponds to the low-Mach stabilizing velocity term defined by $v_{\gamma}$ in section 1.3. As in [2], this term is used only to correct the relative ALE velocity $\boldsymbol{w}$. Thus, contrary to [45], the low-mach stabilizing term (noted here $\boldsymbol{\Gamma}_{i j}$ ) cannot be assimilated to a PST as it does not move the particles. Besides, even if $\gamma-S P H-A L E$ is used in Lagrangian description $\left(v_{0}=v\right)$, if $\gamma \neq 0$ the flux terms in (26) are activated. They can be assimilated as stabilizing terms added in the mass and momentum equations comparatively to standard SPH. It is similar to stabilizing techniques based on diffusive terms $[22,23,21]$ (but made conservative and consistent thanks to the ALE formalism). Particularly, note that if $\gamma \neq 0$ the additional term appearing in the mass equation can be read as $\gamma c_{0} \omega_{i} \sum_{j} \omega_{j}\left(\rho_{j}-\rho_{i}\right)\left\|\boldsymbol{A}_{i j}\right\|$ which is directly similar to the $\delta-S P H$ scheme [32] using the following density dissipative term $2 \delta c_{0} \sum_{j} \omega_{j}\left(\rho_{j}-\rho_{i}\right)\left\|\nabla W_{i j}\right\| \frac{h}{\left\|\boldsymbol{r}_{i j}\right\|}$.

\subsubsection{Standard SPH Formulation}

One can notice that, disregarding $\boldsymbol{\Gamma}_{i j}$ and $\boldsymbol{\pi}_{i j}$, a classical SPH formulation with constant masses $m_{i}=\omega_{i} \rho_{i}$ is recovered by choosing $\boldsymbol{v}_{0}=\boldsymbol{v}$ (purely Lagrangian description)

$$
\left\{\begin{array}{l}
\frac{d \boldsymbol{x}_{i}}{d t}=\boldsymbol{v}\left(\boldsymbol{x}_{i}, t\right) \\
\frac{d m_{i}}{d t}=0 \\
\frac{d \rho_{i}}{d t}=-\rho_{i} \sum_{j} \frac{m_{j}}{\rho_{j}}\left\langle\left(\boldsymbol{v}_{j}-\boldsymbol{v}_{i}\right), \boldsymbol{A}_{i j}\right\rangle \\
\frac{d \boldsymbol{v}_{i}}{d t}=-\sum_{j} m_{j} \frac{\left(p_{i}+p_{j}\right)}{\rho_{i} \rho_{j}} \boldsymbol{A}_{i j}
\end{array}\right.
$$

Actually, in the SPH literature, it can also be found the following equivalent formulation for $\rho$ and $\boldsymbol{v}$

$$
\left\{\begin{array}{c}
\frac{d \rho_{i}}{d t}=-\sum_{j} m_{j}\left\langle\left(\boldsymbol{v}_{j}-\boldsymbol{v}_{i}\right), \boldsymbol{A}_{i j}\right\rangle \\
\frac{d \boldsymbol{v}_{i}}{d t}=-\sum_{j} m_{j}\left(\frac{p_{i}}{\rho_{i}^{2}}+\frac{p_{j}}{\rho_{j}^{2}}\right) \boldsymbol{A}_{i j}
\end{array}\right.
$$




\subsection{Arbitrary Velocity Field}

With an ALE formulation $(25,26)$ the particles are moved following the velocity field $\boldsymbol{v}_{0}$. Such field has the property of being arbitrary in the sense that, as suggested by Vila [1], a smart choice of $\boldsymbol{v}_{0}$ could actually increase both stability and robustness of the calculations without impacting the scheme stability conditions (detailed in section 5). Various methods are available in the literature as Monaghan's XSPH method for instance [26, 7], or also PST [30, 27, 28, 47]. Such methods consist in reorganizing the particles during the calculations to increase the accuracy by preventing the formation of anisotropic spatial particle distributions, tensile instability and particle clustering. In practice here the idea is to take the transport velocity as $\boldsymbol{v}_{0}=\boldsymbol{v}+\boldsymbol{\delta}_{v}$ where the regularizing technique is stored in $\boldsymbol{\delta}_{v}$.

In case of XSPH, particles are moved with a smoother velocity field than the material one to prevent particle penetration. It is achieved by evaluating the difference to the local mean material velocity. According to [26], we implement $\mathrm{XSPH}$ with $\gamma-S P H-A L E$ by taking

$$
\boldsymbol{\delta}_{v X s p h}=\epsilon_{X s p h} \sum_{j} \frac{m_{j}}{\bar{\rho}_{i j}}\left(\boldsymbol{v}_{j}-\boldsymbol{v}_{i}\right) W_{i j}, \text { where } 0 \leq \epsilon \leq 1
$$

$\mathrm{Xu}$ et al. [27] proposed to introduce a slight disorder within the particle distribution thanks to a position shift. Lind et al. [28] proposed a similar technique in the context of incompressible SPH, but interpreted the position shift as a Fick's law like diffusion process. They incorporated as well Monaghan's tensile instability correction [24] to prevent both tensile instability and particle clumping. This position shift technique was recently applied in the context of Euler-Lagrange coupling, to improve the accuracy in the Lagrangian areas $[38,39]$. However, as only particle positions are shifted, the state variables need to be interpolated to limit inconsistencies. Besides, as this regularization technique is a particle diffusion process based on the particle concentration gradient $\nabla C$, a particular treatment has to be done in case of free surface flows. Indeed, a very large $\nabla C$ is expected at the free surface which generates the free surface diffusion. This phenomenon is limited by a control on the normal shifting component at the free surface which is detected thanks to the position gradient [10]. However, such implementation actually violates the momentum conservation. Oger et al. [30] proposed, as suggested by Vila [1], to implement this Fick's law like PST in the ALE context through the transport field $v_{0}$. As introduced in section 1.2, the ALE framework allows to stay conservative and consistent. Contrary to $[27,28,38,39]$, the state variables are now consistently evaluated relatively to PST. In [30] the free surface is detected thanks to Marrone et al. [48] detection algorithm. It uses the minimum eigenvalue $\lambda_{\text {min }}$ of the computed renormalization matrix $B_{i}$ (introduced in section 3.1). However, contrary to [28] the shifting is completely turned off on the free surface. As detailed by Khayyer et al. [31], to allow a slight normal shifting or to completely turn it off at the free surface generates inconsistencies (as particle clustering at the free surface or the presence of a gap between free surface and inner particles). These inconsistencies are generated by an inaccurate evaluation of $\nabla C$ at the free surface. To prevent this, it is proposed in [31] to exactly evaluate the free surface normal vector thanks to $B_{i}$, insuring an exact elimination of the normal shifting component. Sun et al. [32] adapted a similar to [28] PST to enhance the $\delta-S P H$ scheme. Adami et al. [29] proposed an ALE like WCSPH formulation in the sense that it uses an advection velocity. This advection velocity is evaluated thanks to a background pressure gradient. It requires an additional term in the momentum equation. This method is later extended to solids by Zhang et al. [33].

We propose a PST based on the combination of Oger et al. [30], Lind et al. [28], Sun et al. [32] and Khayyer et al. [31] works. It consists in choosing $\boldsymbol{\delta}_{v}$ as :

$$
\boldsymbol{\delta}_{\boldsymbol{v} \boldsymbol{i}}=\left\{\begin{array}{l}
\boldsymbol{\delta}_{v X s p h}-D \boldsymbol{n}_{\boldsymbol{\delta}}, \text { if } D\left\|\boldsymbol{n}_{\boldsymbol{\delta}}\right\|<m_{\delta}\left\|\boldsymbol{v}_{\boldsymbol{i}}\right\| \\
\boldsymbol{\delta}_{v X s p h}-m_{\delta}\left\|\boldsymbol{v}_{\boldsymbol{i}}\right\| \frac{\boldsymbol{n}_{\boldsymbol{\delta}}}{\left\|\boldsymbol{n}_{\boldsymbol{\delta}}\right\|}, \text { otherwise }
\end{array}\right.
$$

with

$$
\boldsymbol{n}_{\boldsymbol{\delta}}=\sum_{j} \omega_{j}\left(1+f_{i j}\right) \boldsymbol{\nabla} \boldsymbol{W}\left(\boldsymbol{x}_{\boldsymbol{i}}-\boldsymbol{x}_{\boldsymbol{j}}, R_{\delta}\right) \text { and } f_{i j}=R_{T}\left(\frac{W_{i j}}{W(\Delta x)}\right)^{n_{T}}
$$

$\left(R_{\delta}, m_{\delta}\right)=(\Delta x, 0.4)$ is the couple of shifting parameters respectively the radius of the kernel support and the shifting magnitude. Note that such expression for $\boldsymbol{\delta}_{v i}$ aims to limit its magnitude to $m_{\delta}\left\|\boldsymbol{v}_{\boldsymbol{i}}\right\|$. $\boldsymbol{n}_{\boldsymbol{\delta}}$ is the particle concentration gradient. $D$ is the diffusion coefficient from the Fick's law defined by $D=2 h_{i} c_{0} M$ where $h$ is the smoothing length, $c_{0}$ the referential sound speed and $M$ the Mach number. Monaghan's [24] tensile instability correction function is described by $f_{i j} .\left(R_{T}, n_{T}\right)=(0.2 ; 4)$ as in $[30,28]$ and $W(\Delta x)$ corresponds to the kernel function evaluated with a radius of $\Delta x$ (initial particle spacing). The free surface is detected similarly to [32] thanks to the minimum eigenvalue of $B_{i}$. Finally, the normal vector is accurately evaluated thanks to $B_{i}$ as 
described in [31]. Thus, the concentration gradient is evaluated by $\boldsymbol{n}_{\delta}$ and defines then a shifting velocity $\boldsymbol{\delta}_{v}$ following (32). If the particle is in an area where $\lambda_{\text {min }} \leq 0.2$ (meaning in particle rarefaction areas or singular point as corners) the shifting is turned off. If the particle is in an area where $0.2 \leq \lambda_{\min } \leq 0.75$ (meaning near the free surface), only tangential shifting is applied. And if $\lambda_{\min }>0.75$ (meaning inside the fluid domain) the total shifting is applied.

Thanks to the ALE framework, a simple treatment of the arbitrary velocity $\boldsymbol{v}_{0}$ is insured. This crucial property allows the implementation of regularizing technique such as PST while preserving the scheme conservativity, stability and consistency.

\section{Nonlinear Stability Analysis}

As detailed in Section 2, the stability of the continuous scheme is achieved thanks to the dissipative behavior generated by the artificial viscosity and the Low-Mach scheme (6). At a discrete level however, the approximation of the continuous scheme (5) generates additional terms (due to space and time discretization) preventing such direct conclusion. Thanks to the following nonlinear stability analysis (similar to the one performed by Grenier et al. [2], Lavalle et al [3] and Couderc et al. [43] in a FV framework), we provide stability conditions on the scheme parameters allowing to control these additional terms. To do so, we detail here that the proposed scheme (26) is actually conservative, robust, stable and consistent.

\subsection{Conservation}

As a first step we can notice that,

Under the conditions

$$
\left\{\begin{array}{l}
\boldsymbol{A}_{i j}=-\boldsymbol{A}_{j i} \\
\boldsymbol{w}_{i j}=\boldsymbol{w}_{j i} \\
\boldsymbol{\pi}_{i j}=-\boldsymbol{\pi}_{j i}
\end{array}\right.
$$

and taking $\Phi_{i}=\left(\begin{array}{c}\omega_{i} \rho_{i} \\ \omega_{i} \rho_{i} \boldsymbol{v}_{i}\end{array}\right)$ we have according to $(26)$

$$
\frac{d}{d t}\left(\sum_{i \in P} \omega_{i} \Phi_{i}\right)=0
$$

which corresponds to the expected conservation condition (21) (with a source term equal to zero).

\subsection{Robustness}

As a second step, we can exhibit a CFL condition density bounds.

Indeed, enforcing the condition

$$
\Delta t \frac{\|\boldsymbol{w}\|_{\infty}}{h} \leq \min _{n, i}\left(N_{\rho}^{i, n}\right)
$$

insures that

$$
\forall t, \rho_{i, \min }(t) \leq \rho_{i}(t) \leq \rho_{i, \max }(t)
$$

We recall that $h$ is the smoothing length (half radius of the kernel support) and $c_{0}$ is the material sound speed. For sake of clarity we refer to Appendix A (52) for the definitions of $N_{\rho}^{i, n}$ and for the detailed proof. We have $\|\boldsymbol{w}\|_{\infty}=\sup _{i}\left\|\boldsymbol{w}_{i}\right\|$ and

$$
\left\{\begin{array} { c } 
{ \rho _ { i , \operatorname { m i n } } ( t ) = \rho _ { i } ^ { 0 } e ^ { - C _ { \operatorname { m i n } } t } } \\
{ \rho _ { i , \operatorname { m a x } } ( t ) = \rho _ { i } ^ { 0 } e ^ { C _ { \operatorname { m a x } } t } }
\end{array} \text { and } \left\{\begin{array}{l}
C_{\min }=\frac{1}{\Delta t} \ln \left(2 \frac{\omega_{\max }}{\omega_{\min }}\right) \\
C_{\text {max }}=\frac{1}{\Delta t} \ln \left(\frac{3}{2} \frac{\omega_{\max }}{\omega_{\min }}\right)
\end{array}\right.\right.
$$

Density Bounds We thus have the existence of density bounds $\left(\rho_{\min }, \rho_{\max }\right)$ and we set $\eta=\frac{\rho_{\max }}{\rho_{\min }}$ the maximum density ratio.

Interpretation Classical CFL conditions are defined as $\Delta t \frac{c_{0}}{h} \leq N_{C F L}$ where $N_{C F L} \leq 1$ is a safety coefficient ensuring the stability of the scheme. 
- In the same way, we can also approximate condition $\Delta t \frac{\|\boldsymbol{w}\|_{\infty}}{h} \leq N_{\rho}$ by $\Delta t \leq \frac{h}{C \eta\left(\|\boldsymbol{w}\|_{\infty}+\frac{\gamma}{2 c_{0}}(\eta-1)\right)}$ where $C$ is defined as (16). In a weakly-compressible framework (meaning that $\eta \simeq 1,\|\boldsymbol{w}\|_{\infty} \ll c_{0}$ ), this condition is reduced to $\Delta t \frac{\|\boldsymbol{w}\|_{\infty}}{h} \leq \frac{1}{C}$.

- Note that $C$ (16) stays bounded both in regular or arbitrary particle distributions. See Section 6.3 fig. 7 and [19] for an analysis in the regular case.

In practice, condition (34) is less restrictive than a classical CFL condition.

\subsection{Energy Balance}

We now bring interest on the energy stability of the proposed scheme (26). Meaning that we want a control on its total energy noted $\mathcal{E}_{n}=\sum_{i \in P} \omega_{i}^{n} E_{i}^{n}$. We define the total energy $E$ as $E=\frac{1}{2} \rho\|v\|^{2}+\varphi$ where $\varphi=\rho \int_{\rho_{0}}^{\rho} \frac{p(r)}{r^{2}} d r$ is the total free energy and corresponds to the barotropic pressure. As introduced in section 1.1, we use a linear EOS defined by $p=c_{0}^{2}\left(\rho-\rho_{0}\right)$. To achieve such a control, the idea is to complete on the discrete scheme (26), a similar process to the one performed on the continuous modified scheme $(5,6)$ detailed in Section 2 :

1. An energy balance is performed on the scheme (26), and the production terms are exhibited as follows

$$
\sum_{i \in P}\left[w_{i}^{n+1} E_{i}^{n+1}-w_{i}^{n} E_{i}^{n}\right]=\sum_{i \in P}\left[\Delta t S_{i}^{n}+w_{i}^{n+1}\left(Q_{i}^{n}+R_{i}^{n}\right)\right]
$$

with

$$
\begin{aligned}
w_{i}^{n+1} Q_{i}^{n}= & \frac{1}{2} \rho_{i}^{n+1}\left\|\boldsymbol{v}_{\boldsymbol{i}}^{n+\mathbf{1}}-\boldsymbol{v}_{\boldsymbol{i}}^{n}\right\|^{2} \\
& +\Delta t . w_{i}^{n} \sum_{j}\left\langle\mathbf{w}_{i j}^{n}, \boldsymbol{A}_{i j}^{n}\right\rangle \rho_{j}^{n} \frac{1}{2}\left\|\boldsymbol{v}_{j}^{n}-\boldsymbol{v}_{\boldsymbol{i}}^{n}\right\|^{2} w_{j}^{n} \\
& -\Delta t . w_{i}^{n} \sum_{j} \gamma_{i j}^{n}\left(p_{j}^{n}-p_{i}^{n}\right)^{2}\left\|\boldsymbol{A}_{i j}^{n}\right\| w_{j}^{n} \\
w_{i}^{n+1} R_{i}^{n}= & \frac{1}{2} \widetilde{\kappa}_{i}^{\prime n}\left(\rho_{i}^{n+1}-\rho_{i}^{n}\right)^{2} \\
& +\Delta t . w_{i}^{n} \sum_{j} \frac{1}{2}\left\langle\mathbf{w}_{i j}^{n}, \boldsymbol{A}_{i j}^{n}\right\rangle \widetilde{\kappa}_{i j}^{\prime n}\left(\rho_{i}^{n}-\rho_{j}^{n}\right)^{2} w_{j}^{n} \\
& -\Delta t . w_{i}^{n} \sum_{j} \alpha_{i j}^{n}\left\|\boldsymbol{v}_{j}^{n}-\boldsymbol{v}_{\boldsymbol{i}}^{n}\right\|^{2} w_{j}^{n} \\
\Delta t S_{i}^{n}=-p_{i}^{n}[ & {\left.\left[w_{i}^{n+1}-w_{i}^{n}\right)-2 \Delta t w_{i}^{n} \sum_{j}\left\langle\boldsymbol{v}_{0 i j}^{n}, \boldsymbol{A}_{i j}^{n}\right\rangle w_{j}^{n}\right] }
\end{aligned}
$$

where $\boldsymbol{v}_{0 i j}^{n}=\frac{1}{2}\left(\boldsymbol{v}_{0 i}^{n}+\boldsymbol{v}_{0 j}^{n}\right)$. Also, with the definition of $\kappa=\frac{d \varphi}{d \rho}$, a Taylor's expansion of $\varphi$ gives some $\left(\theta_{i j}, \theta_{i}\right) \in[0,1]^{2}$ such that $\tilde{\kappa}_{i j}^{n \prime}=\kappa^{\prime}\left(\left(1-\theta_{i j}\right) \rho_{i}^{n}+\theta_{i j} \rho_{j}^{n}\right)$ and $\tilde{\kappa}_{i}^{n \prime}=\kappa^{\prime}\left(\left(1-\theta_{i}\right) \rho_{i}^{n}+\theta_{i} \rho_{i}^{n+1}\right)$ with

$$
\left\{\begin{array}{l}
\varphi\left(\rho_{j}^{n}\right)-\varphi\left(\rho_{i}^{n}\right)=\kappa_{i}^{n}\left(\rho_{j}^{n}-\rho_{i}^{n}\right)+\frac{1}{2} \tilde{\kappa}_{i j}^{n \prime}\left(\rho_{j}^{n}-\rho_{i}^{n}\right)^{2} \\
\varphi\left(\rho_{i}^{n+1}\right)-\varphi\left(\rho_{i}^{n}\right)=\kappa_{i}^{n}\left(\rho_{i}^{n+1}-\rho_{i}^{n}\right)+\frac{1}{2} \tilde{\kappa}_{i}^{n \prime}\left(\rho_{i}^{n+1}-\rho_{i}^{n}\right)^{2}
\end{array}\right.
$$

Note that the discrete counterparts $s_{\gamma}(38)$ and $s_{\alpha}(39)$ of the dissipative source terms $S_{\gamma}(7)$ and $S_{\alpha}(8)$ are obtained in, respectively, (35), and (36) and defined as

$$
\begin{gathered}
s_{\gamma}=-\sum_{n i j} \Delta t w_{i}^{n} w_{j}^{n} \gamma_{i j}^{n}\left(p_{j}^{n}-p_{i}^{n}\right)^{2}\left\|\boldsymbol{A}_{i j}^{n}\right\| \\
s_{\alpha}=-\sum_{n i j} \Delta t w_{i}^{n} w_{j}^{n} \alpha_{i j}^{n}\left\|\boldsymbol{v}_{j}^{n}-\boldsymbol{v}_{\boldsymbol{i}}^{n}\right\|^{2}
\end{gathered}
$$

2. The production terms $(35,36,37)$ are now estimated and gathered to provide a global estimation of the energy production

$$
\begin{aligned}
\mathcal{E}_{n+1}-\mathcal{E}_{n} \leq & \theta \mathcal{E}_{n}+B_{\eta} \\
& +q^{n}(\gamma) \Delta t \sum_{i}\left(w_{i}^{n} \sum_{j}\left[\frac{\widetilde{\kappa}_{i j}^{\prime n}}{2} c_{0}\left(\rho_{j}^{n}-\rho_{i}^{n}\right)^{2}\left\|\boldsymbol{A}_{i j}^{n}\right\| w_{j}^{n}\right]\right) \\
& +r^{n}(\alpha) \Delta t \sum_{i}\left(w_{i}^{n} \sum_{j}\left[\frac{\rho_{j}^{n}}{2} c_{0}\left\|\boldsymbol{v}_{j}^{n}-\boldsymbol{v}_{i}^{n}\right\|^{2}\left\|\boldsymbol{A}_{i j}^{n}\right\| w_{j}^{n}\right]\right)
\end{aligned}
$$

where $\theta=K_{1} \Delta t$ and $\beta=K_{2} \Delta t+K_{3} \Delta t^{2} . K_{1}, K_{2}, K_{3}$ are constants expressed in function of $c_{0},\left\|\boldsymbol{v}_{0}\right\|_{\infty}$ and the total mass. $q$ and $r$ are quadratic functions of $\gamma$ and $\alpha$ respectively. See Appendix B for more details. 
3. A negativity condition on $q$ and $r$ provides a CFL $N_{E}$ and bounds $\left[\alpha_{\min }, \alpha_{\max }\right],\left[\gamma_{\min }, \gamma_{\max }\right]$ giving that

$$
\text { for } \Delta t \frac{c_{0}}{h} \leq N_{E}, \forall(\alpha, \gamma) \in\left[\alpha_{\min }, \alpha_{\max }\right] \mathrm{x}\left[\gamma_{\min }, \gamma_{\max }\right] /\left\{\begin{array}{l}
q \leq 0 \\
r \leq 0
\end{array}\right.
$$

See below for the interpretation. The remaining production terms are evaluated under such stability conditions (40) and the energy balance is reduced to

$$
\mathcal{E}_{n+1} \leq(1+\theta) \mathcal{E}_{n}+B_{\eta}
$$

4. The Gronwall theorem provides then a finite energy bound noted $\mathcal{E}_{T}(41)$ achieving the expected stability.

$$
\exists \mathcal{E}_{T} \geq 0, \forall 0 \leq t \leq T, \mathcal{E}(t) \leq \mathcal{E}_{T}
$$

The proof in the case of a first order time integrator is detailed in Appendix B.

We recall that $h$ is the smoothing length (half radius of the kernel support) and $c_{0}$ is the material sound speed. For sake of clarity we refer to Appendix B $(56,57,58,59)$ for the definitions of $N_{E}, \gamma_{\min }, \gamma_{\max }, \alpha_{\min }, \alpha_{\max }$ and $\mathcal{E}_{T}$.

\section{Interpretation}

- Stability Limit: We define $N_{E}=\frac{1}{C} \min _{n}\left(N_{\alpha}^{n}, N_{\gamma}^{n}\right)$ as the maximum admissible CFL to achieve the scheme stability. $N_{\alpha}^{n}$ and $N_{\gamma}^{n}$ are defined as (56) and, as introduced in section 5.2, $C$ (16) stays bounded around unity both in regular and arbitrary particle distributions (See Section 6.3 fig. 7 and [19] for an analysis in the regular case). Taking $\Delta t \frac{c_{0}}{h}=N_{E}$ gives critical values $\gamma_{c r i t}=\alpha_{c r i t}=\frac{1}{8 \eta^{2} C N_{E}}$ insuring the scheme stability. We recall that $\eta=\frac{\max _{n, i} \rho_{i}^{n}}{\min _{n, i} \rho_{i}^{n}}$. Now in the low-Mach $\operatorname{limit}(M \rightarrow 0, \eta=1)$ we get

$$
\left\{\begin{array}{l}
N_{E}=\frac{\sqrt{2}}{8 C} \text { giving } \Delta t=\frac{\sqrt{2}}{8 C} \frac{h}{c_{0}} \\
\gamma_{c r i t}=\alpha_{\text {crit }}=\frac{1}{\sqrt{2}}
\end{array}\right.
$$

However these values correspond to a stability limit and do not minimize the amount of dissipation generated by the artificial viscosity (28) and the Low-Mach scheme (30) (which is the expected behavior).

- Stability Field: Taking now $N=\Delta t \frac{c_{0}}{h}<N_{E}$, according to (40) we can exhibit stability intervals for $\alpha$ and $\gamma$. To minimize the dissipation induced in the scheme we choose to work with the minimum values. In particular, as detailed in Appendix B, we can theoretically achieve the stability in the low-Mach limit for

$$
\left\{\begin{array}{l}
N=\Delta t \frac{c_{0}}{h}<N_{E} \\
(\alpha, \gamma) \in\left[4 C N ; \frac{1}{8 C N}\right]^{2}
\end{array}\right.
$$

Note that in [2], the stability condition on the low-Mach parameter can be read as $\gamma_{e} \geq \frac{\Delta t}{2 h \rho}$ where $\gamma_{e}$ has the dimension of $\frac{1}{\rho c_{0}}$. According to (43), as the present $\gamma$ is dimensionless, if we set $\gamma_{\min }=4 C N$, we have $\frac{\gamma_{m i n}}{\rho c_{0}}=4 C \frac{\Delta t}{\rho h}$ which is a similar stability condition as the one achieved in $[2,3,45]$. Also, there is a coupling between $\alpha$ and $\gamma$ modifying the stability behavior for extreme values, but this phenomenon is not studied in this work. Thus, in practice, we actually work with $\alpha_{\min }$ and find then $\gamma_{\min }$ after some calculations.

Choice of $\boldsymbol{v}_{0}$ The stability conditions are defined regardless the expression of $\boldsymbol{v}_{0}$ (as soon as it is taken as regular enough) revealing its arbitrary property previously introduced. (40) depends on a geometrical parameter noted $C$ (16). Thus, this condition ensures the scheme stability while the particle distribution stays regular enough. Then it is crucial to be allowed to implement a conservative and consistent regularizing technique through $\boldsymbol{v}_{0}$ (as PST).

Time Step Restriction Here we can anticipate that (40) is not optimal. Indeed, firstly, the optimality is slightly lost due to Jensen's inequality use during the evaluation process. Secondly, these stability conditions ensure the stability of a first order time integration scheme, whereas a second order scheme is used. As detailed in section 4.1 [46] (formally proved in Appendix B), using RK2H time integration scheme preserves the stability achieved by (40). It has the property to relax the condition on the time step. Couderc et al. [43] achieved similar conclusions during the nonlinear stability analysis of a FV low-Mach scheme. 
Additional Estimation Result In addition of result (41) we can exhibit the following estimates by enforcing more restrictive stability conditions (meaning a lower CFL)

$$
\exists\left(\mathcal{E}_{1}, \mathcal{E}_{2}\right) \in \mathbb{R}^{+, 2} /\left\{\begin{array}{l}
\sum_{n, i, j} w_{i}^{n} w_{j}^{n}\left(\rho_{j}^{n}-\rho_{i}^{n}\right)^{2}\left\|\boldsymbol{A}_{i j}^{n}\right\| \Delta t \leq \mathcal{E}_{1} \\
\sum_{n, i, j} w_{i}^{n} w_{j}^{n}\left\|\boldsymbol{v}_{j}^{n}-\boldsymbol{v}_{i}^{n}\right\|^{2}\left\|\boldsymbol{A}_{i j}^{n}\right\| \Delta t \leq \mathcal{E}_{2}
\end{array}\right.
$$

Such estimates are useful to ensure the scheme consistency detailed in Section 5.4. See Appendix B for the establishment of these estimates.

\subsection{Consistency}

Finally, as regards the consistency of the proposed scheme, we refer to theorem 2.2.1 of section 3.2. Note that, according to the proposed equation set $(26)$, we use a different derivative operator than $D_{h}$. It means that not only theorem condition 3 has to be enforced, but theorem condition 2 as well. To avoid this last condition, we can in fact rewrite the proposed scheme as (20) with additional terms stored in the residual $R_{h}(\Phi)$. Thus, we just have to enforce

$$
\forall \varphi \in\left[\mathcal{C}_{0}\left(\mathbb{R}^{d} \mathbb{X}^{+, *}\right)\right]^{p}, \lim _{h} \text { and } \Delta x \rightarrow 0 \int_{\mathbb{R}^{+}}\left(R_{h}\left(\bar{\Phi}^{\triangle}\right), \varphi\right)_{h} d t=0
$$

Mass Residuals We firstly refer to (26-Mass Equation)

$$
\rho_{i}^{n+1} w_{i}^{n+1}=\rho_{i}^{n} w_{i}^{n}-\Delta t w_{i}^{n} \sum_{j} w_{j}^{n}\left(\rho_{j}^{n}+\rho_{i}^{n}\right)\left\langle\boldsymbol{w}_{i j}^{n} \cdot \boldsymbol{A}_{i j}^{n}\right\rangle
$$

According to (20), we extract the following mass residuals

$$
\begin{aligned}
R_{h}(\omega \rho)_{i}= & \sum_{j} w_{j}^{n} \frac{1}{2}\left\langle\left(\rho_{j}^{n}-\rho_{i}^{n}\right)\left(\boldsymbol{w}_{j}^{n}-\boldsymbol{w}_{i}^{n}\right), \boldsymbol{A}_{i j}^{n}\right\rangle \\
& +\sum_{j} w_{j}^{n} \gamma_{i j}^{n}\left(p_{j}^{n}-p_{i}^{n}\right)\left(\rho_{j}^{n}+\rho_{i}^{n}\right)\left\|\boldsymbol{A}_{i j}^{n}\right\|
\end{aligned}
$$

Momentum Residuals We secondly refer to (26-Momentum Equation)

$$
\rho_{i}^{n+1} w_{i}^{n+1} \boldsymbol{v}_{i}^{n+1}=\rho_{i}^{n} w_{i}^{n} \boldsymbol{v}_{i}^{n}-\Delta t w_{i}^{n} \sum_{j} w_{j}^{n}\left(\rho_{j}^{n} \boldsymbol{v}_{j}^{n}+\rho_{i}^{n} \boldsymbol{v}_{i}^{n}\right)\left\langle\boldsymbol{w}_{i j}^{n} \cdot \boldsymbol{A}_{i j}^{n}\right\rangle-\Delta t w_{i}^{n} \sum_{j} w_{j}^{n}\left(p_{i j}^{n} \boldsymbol{A}_{i j}^{n}-\boldsymbol{\pi}_{i j}^{n}\right)
$$

According to (20), we extract the following momentum residuals

$$
\begin{aligned}
R_{h}(\omega \rho \boldsymbol{v})_{i}= & \sum_{j} w_{j}^{n} \frac{1}{2}\left(\rho_{j}^{n} \boldsymbol{v}_{j}^{n}-\rho_{i}^{n} \boldsymbol{v}_{i}^{n}\right)\left\langle\left(\boldsymbol{w}_{j}^{n}-\boldsymbol{w}_{i}^{n}\right), \boldsymbol{A}_{i j}^{n}\right\rangle \\
& +\sum_{j} w_{j}^{n} \gamma_{i j}^{n}\left(p_{j}^{n}-p_{i}^{n}\right)\left(\rho_{j}^{n} \boldsymbol{v}_{j}^{n}+\rho_{i}^{n} \boldsymbol{v}_{i}^{n}\right)\left\|\boldsymbol{A}_{i j}^{n}\right\| \\
& +2 \sum_{j} w_{j}^{n} \alpha_{i j}^{n}\left(\boldsymbol{v}_{j}^{n}-\boldsymbol{v}_{i}^{n}\right)
\end{aligned}
$$

\section{Residuals Estimation}

Using

- the density bounds $\left(\rho_{\min }, \rho_{\max }\right)$ of section 5.2 ,

- the energy bound (41) of section 5.3,

- the consistency estimates (44) of section 5.3,

- the symmetry properties of the mass (46) and momentum (47) residuals of section 5.4,

we can actually enforce the consistency condition (45), ensuring then the weak consistency of the proposed scheme (26), with the model PDE (22), in the sense of (18).

The proof is not detailed in this paper but consists in applying the technique exposed by Vila in [1].

\section{Validation}

Knowing the conditions ensuring that the proposed $\gamma-S P H-A L E$ scheme is stable, we now validate it on several test cases widely studied in the SPH literature: isentropic shock tube, rotating square patch of fluid, oscillating drop and dam break. 


\subsection{Remarks on the stability in practice}

As introduced in section 5.3, the stability conditions $(42,43)$ are very restrictive due to the first order time integrator and corresponding to the asymptotic limit. In practice, we note that working with a second order scheme RK2H (detailed in section 4.1) allows to relax the time step restriction. Indeed, for the following test cases, using RK2H with a CFL classically used in the SPH literature $N_{0.3}=0.3$ [7] also provides stability intervals. It means that for a given CFL $N_{C F L}$ we can find $\gamma^{-}, \gamma^{+}, \alpha^{-}$and $\alpha^{+}$in $\mathbb{R}^{+}$such that

$$
\text { For } \Delta t=N_{C F L} \frac{h}{c_{0}}, \exists(\alpha, \gamma) \in\left[\alpha^{-} ; \alpha^{+}\right] \mathrm{x}\left[\gamma^{-} ; \gamma^{+}\right] \text {ensuring the scheme stability. }
$$

RK2H not only relaxes the time step restriction, but also preserves the theoretical stability pattern. Thus, according to section 5.3 (43) and Appendix B results, with RK2H if we set the following constants

$$
\left\{\begin{array} { c } 
{ f _ { \gamma } ^ { - } = \frac { \gamma ^ { - } } { N _ { C F L } } } \\
{ f _ { \gamma } ^ { + } = \gamma ^ { + } N _ { C F L } }
\end{array} \text { and } \left\{\begin{array}{c}
f_{\alpha}^{-}=\frac{\alpha^{-}}{N_{C F L}} \\
f_{\alpha}^{+}=\alpha^{+} N_{C F L}
\end{array}\right.\right.
$$

we can define the stability intervals as it follows

$$
\forall N \text { admissible, }\left\{\begin{array} { c } 
{ \gamma _ { \operatorname { m i n } } = f _ { \gamma } ^ { - } N } \\
{ \gamma _ { \operatorname { m a x } } = \frac { f _ { \gamma } ^ { + } } { N } }
\end{array} \text { and } \left\{\begin{array}{c}
\alpha_{\min }=f_{\alpha}^{-} N \\
\alpha_{\max }=\frac{f_{\alpha}^{+}}{N}
\end{array} \text { where } N=\frac{\Delta t c_{0}}{h}\right.\right.
$$

The stability intervals corresponding to the CFL $N$ can then be recovered (in function of the intervals obtained with $\left.N_{C F L}\right)$.

\subsection{Isentropic shock tube}

The isentropic shock tube is a classical academic test case with a known exact solution. A large number of configurations and data is available in the literature allowing to efficiently evaluate $\gamma-S P H-A L E$ in a compressible framework. We focus here on a barotropic shock tube using $p=c_{0}^{2}\left(\rho-\rho_{0}\right)$ as EOS. Marongiu [49] and Leduc [50] performed similar test cases to evaluate SPH Riemann Solvers.

We choose the case of a pressure discontinuity generated by the following density field:

$$
\left\{\begin{array} { l } 
{ \rho _ { L } = 1 1 0 0 } \\
{ v _ { L } = 0 }
\end{array} \text { and } \left\{\begin{array}{l}
\rho_{R}=1000 \\
v_{R}=0
\end{array}\right.\right.
$$

$\mathrm{L}$ and $\mathrm{R}$ define respectively the left and right parts of the domain with respect to the discontinuity initial position. The fluid is taken as having a referential density $\rho_{0}=1000 \mathrm{~kg} / \mathrm{m}^{3}$ and a sound speed $c_{0}=1466 \mathrm{~m} / \mathrm{s}$. The tube is taken with a length $L_{x}=0.1 \mathrm{~m}$ and a width $L_{y}=0.01 \mathrm{~m}$. The discontinuity is initially defined at $x=0.05 \mathrm{~m}$. All computations are performed with cubic B-splines kernel functions and a fixed smoothing length $h=1.6 \Delta x$, where $\Delta x$ is the initial particle spacing. The exact solution used as a reference is given by an exact 1D Riemann solver with 2000 cells. SPH computations are performed in 2D with periodic boundary conditions to mimic a 1D case. We present the results given by the RSALE (SPH ALE HLLC Riemann Solver with a MUSCL scheme and a Sweby slope limiter $\beta=1$ ), and by $\gamma-S P H-A L E$. The initial spacing is set to $\Delta x=2 \cdot 10^{-4} \mathrm{~m}$. The initial discontinuity generates a left rarefaction wave and a right shock wave. The solution is then characterized by three pressure levels $\left(2.1492 .10^{8} \mathrm{~Pa}, 1.0493 .10^{8} \mathrm{~Pa}, 0 \mathrm{~Pa}\right)$ and two velocity levels $(0 \mathrm{~m} / \mathrm{s}, 69.892 \mathrm{~m} / \mathrm{s})$. The velocity results are not presented in this paper as they generate the same observations as the pressure results.

Regarding $\gamma-S P H-A L E$ stability, as introduced in section 6.1 , the stability limit $\alpha=\gamma=0.7$ (42) is too dissipative and is not optimal. Indeed, $\alpha=0.7$ induces too much artificial viscosity drastically diffusing the discontinuities (see Figure 1). To find the optimal parameters a practical stability study is achieved to exhibit the stability diagram $(\alpha, \gamma)$. Two stability criterion are taken into account: 1- For high values of $(\alpha, \gamma)$ nonphysical behavior leading to simulation crash, 2- For small values of $(\alpha, \gamma)$ spurious pressure and velocity oscillations on the intermediate level. We deduce the following referential stability intervals

$$
\left\{\begin{array}{l}
N_{C F L}=0.9 N_{0.3} \\
\gamma \in[0.05 ; 3.1] \\
\alpha \in[0.0 ; 1.5]
\end{array}\right.
$$

According to section 6.1 remark, we can set

$$
\left\{\begin{array} { c } 
{ f _ { \gamma } ^ { - } = \frac { 0 . 0 5 } { N _ { C F L } } } \\
{ f _ { \gamma } ^ { + } = 3 . 1 N _ { C F L } }
\end{array} \text { and } \left\{\begin{array}{c}
f_{\alpha}^{-}=0.0 \\
f_{\alpha}^{+}=1.5 N_{C F L}
\end{array}\right.\right.
$$




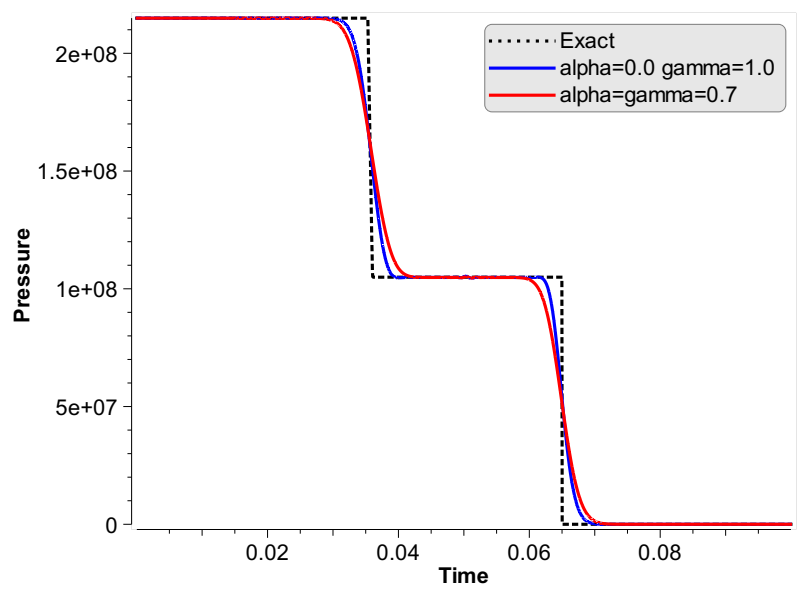

Figure 1: Shock Tube Comparison of $\gamma-S P H-A L E$ stability limit (red) and optimal parameter choice (blue) with the exact solution (black dotted line)

\begin{tabular}{|c|c|c|}
\hline $\mathbf{N}$ & $0.5 N_{0.3}$ & $0.3 N_{0.3}$ \\
\hline \hline$\gamma_{\exp }$ & {$[0.03 ; 5.5]$} & {$[0.03 ; 9.1]$} \\
\hline $\boldsymbol{\alpha}_{\exp }$ & {$[0.0 ; 2.7]$} & {$[0.0 ; 4.6]$} \\
\hline
\end{tabular}

\begin{tabular}{|c|c|c|}
\hline $\mathbf{N}$ & $0.5 N_{0.3}$ & $0.3 N_{0.3}$ \\
\hline \hline$\gamma_{t h}$ & {$[0.028 ; 5.58]$} & {$[0.017 ; 9.3]$} \\
\hline $\boldsymbol{\alpha}_{t h}$ & {$[0.0 ; 2.7]$} & {$[0.0 ; 4.5]$} \\
\hline
\end{tabular}

Table 1: Shock Tube Stability Intervals in function of CFL $N$ : experimental results (left), theoretical results in sense of section 6.1 (right)

After some calculations, we extract the experimental stability intervals (subscript "exp") for cases $N=0.5 N_{0.3}$ and $N=0.3 N_{0.3}$ listed in Table 1-Left. Table 1-Right presents the predicted intervals (subscript "th") using section 6.1 remark. We can see that both experimental and theoretical stability intervals are in very good agreements allowing to faithfully predict the stability intervals for any given CFL. As introduced in section 5.3, in practice we want to minimize the amount of artificial dissipation in the scheme. Then we choose to work with $\alpha_{\min }=0.0$ and then, we find $\gamma_{\min }=1.0$ after some calculations.

Figure 2 shows the comparison between exact solution, Standard SPH (in the sense of section 4.2 .3 in $2 \mathrm{D}$ with standard artificial viscosity $\alpha_{\text {Monaghan }}=1.5[5]$ ), RSALE and $\gamma-S P H-A L E$ with $\alpha=0.0$ and $\gamma=1.0$ in a purely Lagrangian framework $\left(v_{0}=v\right.$ then no PST). Standard SPH manages to reproduce the pressure jumps by diffusing the discontinuities over a few particles. A significant oscillation also appears at the discontinuity initial position $x=0.05 \mathrm{~m}$ (Figure $2 \mathrm{left}$ ). As expected, RSALE faithfully reproduces the pressure jumps. Both discontinuities are less diffused than with standard SPH but overshoots appear. There is also a slight oscillation at the discontinuity initial position but again less significant than with standard SPH. Regarding $\gamma-S P H-A L E$, both discontinuities are diffused similarly to standard SPH (no overshoots). Also, an oscillation appears at $x=0.05 \mathrm{~m}$ but controlled similarly to RSALE. Even if the discontinuities are captured more accurately with RSALE (narrower jumps), in counterpart it generates pressure overshoots. This behavior is controlled by the slope limiter. Here the Sweby limiter is used with $\beta=1$ (minimum threshold) and do not prevent the overshoots. Other limiters are available in the literature and may be more suited for this case (see [40]). Regarding the slight oscillation at the discontinuity initial position, it comes from the purely Lagrangian framework. No regularization of the particle distribution is allowed, and the initial disorder induced by the discontinuity is not regularized. However, RSALE and $\gamma-S P H-A L E$ manages to limit the oscillation amplitude and stay in very good agreements with the exact solution contrary to Standard SPH.

$\gamma-S P H-A L E$ results in Lagrangian description for different $\gamma$ are presented on Figure 3. We can see that $\gamma<1.0$ provides narrower jumps but also generates overshoots and oscillations (like RSALE without slope limiter). Then, increasing $\gamma$ stabilizes the scheme by damping the spurious oscillations. It allows to efficiently control $\gamma-S P H-A L E$ stability and accuracy. Note that $\gamma=1.0$ is the threshold below which the scheme is unstable (presence of overshoots and oscillations).

We compare now on figure 4, the pressure fields obtained by RSALE and $\gamma-S P H-A L E$ with $\gamma=1$ firstly in purely Eulerian description $\left(\boldsymbol{v}_{0}=\mathbf{0}\right.$, figure 4 top) and secondly by using the PST introduced in section 4.3 ( $v_{0}$ arbitrary, figure4 bottom ). In both cases we can see that $\gamma-S P H-A L E$ faithfully reproduces the RSALE results in very good agreements with the exact solution. Note that thanks to PST no deviation appears at the discontinuity initial position (figure4 bottom). The disorder initially produced by the discontinuity is regularized. Such deviation does not appear in the Eulerian case as particles are not moved $\left(v_{0}=0\right)$. 

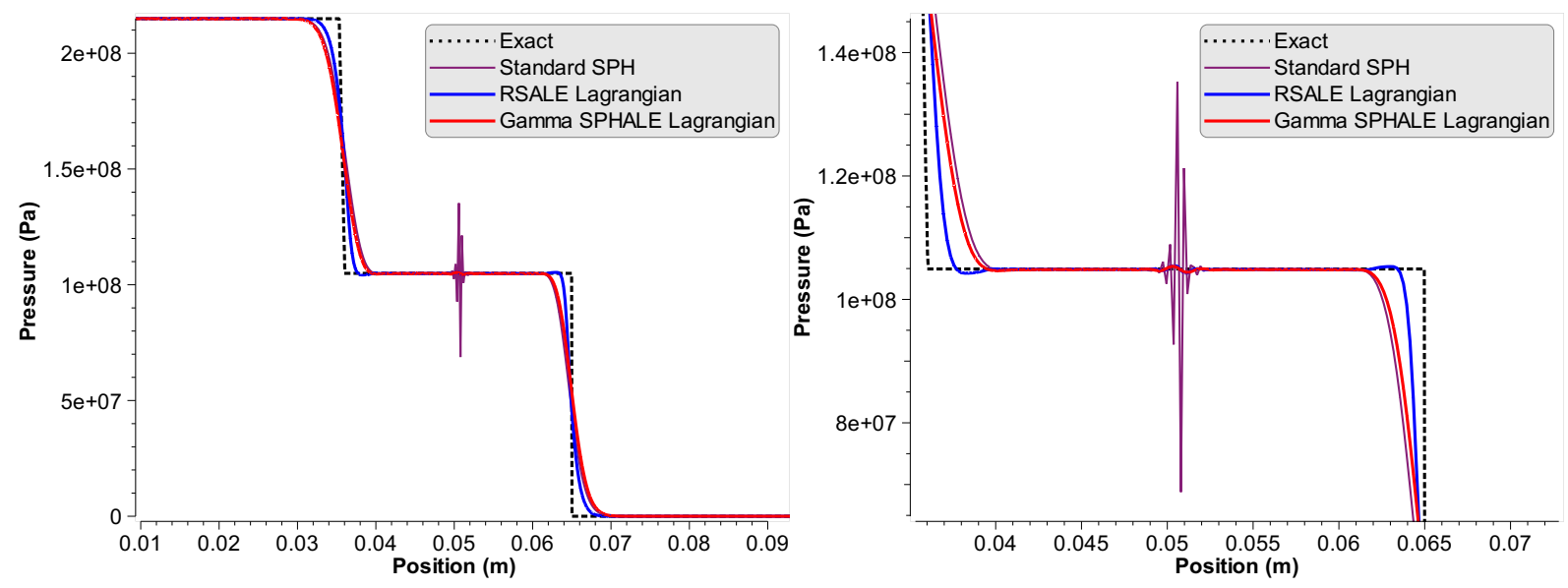

Figure 2: Shock Tube Comparison between exact solution (black dotted line), Standard SPH (purple), RSALE (blue) and $\gamma-S P H-A L E$ with $\gamma=1$ (red), pressure fields at $t=10^{-5} \mathrm{~s}$. Left: global solution, Right: zoom on the intermediate pressure level

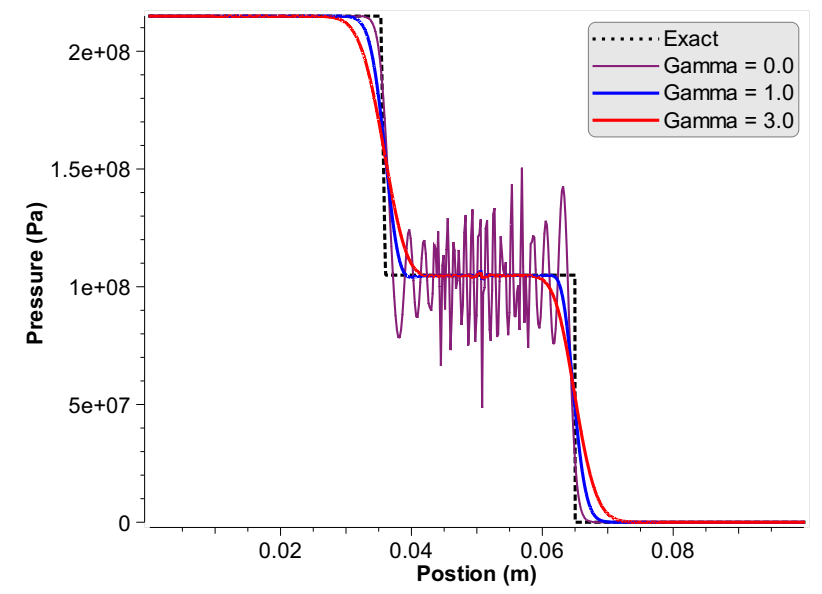

Figure 3: Shock Tube Comparison between exact solution (black dotted line) and $\gamma-S P H-A L E$ (purple: $\gamma=$ 0 , blue: $\gamma=1.0$, red: $\gamma=3.0$ ) Pressure fields at $t=10^{-5} \mathrm{~s}$
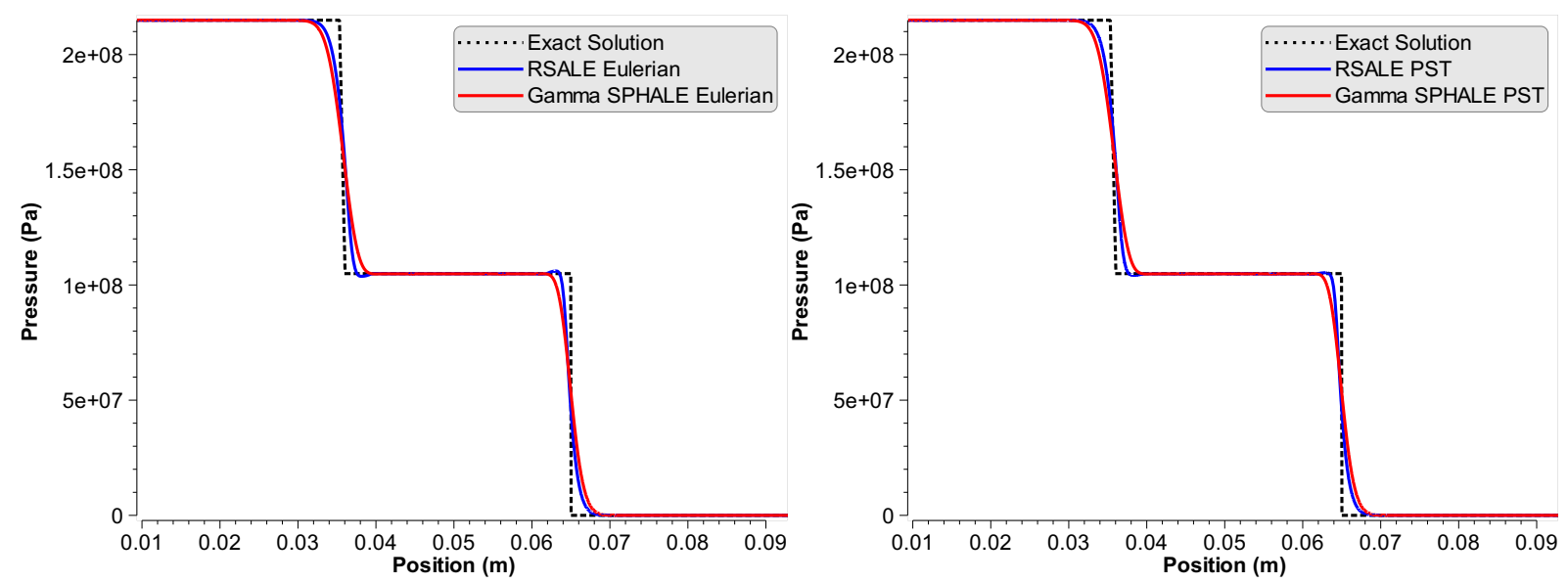

Figure 4: Shock Tube Comparison between the pressure fields obtained by RSALE and $\gamma-S P H-A L E$ $(\gamma=1.0, \alpha=0.0)$ in Eulerian formulation (left) and with PST (right) at $t=10^{-5} \mathrm{~s}$ 


\begin{tabular}{|c|c|c|}
\hline $\mathbf{N}$ & $0.5 N_{0.3}$ & $0.3 N_{0.3}$ \\
\hline \hline$\gamma_{\exp }$ & {$[0.001 ; 3.8]$} & {$[0.0 ; 7.8]$} \\
\hline $\boldsymbol{\alpha}_{\exp }$ & {$[0.007 ; 2.3]$} & {$[0.004 ; 3.6]$} \\
\hline
\end{tabular}

\begin{tabular}{|c|c|c|}
\hline $\mathbf{N}$ & $0.5 N_{0.3}$ & $0.3 N_{0.3}$ \\
\hline \hline$\gamma_{t h}$ & {$[0.0011 ; 4.14]$} & {$\left[6.7 .10^{-4} ; 6.9\right]$} \\
\hline $\boldsymbol{\alpha}_{t h}$ & {$[0.0056 ; 2.16]$} & {$[0.0033 ; 3.6]$} \\
\hline
\end{tabular}

Table 2: Rotating Square Stability Intervals in function of CFL $N$ : experimental results (left), theoretical results in the sense of section 6.1 (right)

$\gamma-S P H-A L E$ provides good results in terms of accuracy comparatively to RSALE and exact solution in each formulations (Lagrangian, Eulerian and with PST). The low-Mach scheme appears as an efficient tool to prevent overshoots and spurious oscillations. $\gamma-S P H-A L E$ gives then a reliable compressible solution without the complexity induced by the resolution of Riemann problems and the use of slope limiters.

\subsection{Rotating square patch of fluid}

A challenging benchmark test proposed by Colagrossi [51] is studied in this section. It is based on a $2 \mathrm{D}$ square patch of fluid free from any external forces and following a rotating initial velocity field. Such case is similarly treated by Oger et al. [30],Sun et al. [32] or Khayyer et al. [31].

The numerical domain $\Omega$ is a patch of length $\mathrm{L}$, centered at the origin and subjected to the initial velocity field set as

$$
\left\{\begin{array}{l}
v_{x}=+\omega y \\
v_{y}=-\omega x
\end{array}\right.
$$

Where $\omega$ is the pure rigid rotation velocity. The initial pressure field $p_{0}$ is taken as the solution of the Poisson equation:

$$
\nabla^{2} p_{0}=2 \rho \omega^{2} \text { with } \forall x \in \partial \Omega, p_{0}(x)=0
$$

Giving:

$$
p_{0}(x, y)=\rho \sum_{m=1}^{\infty} \sum_{n=1}^{\infty}-\frac{32 \omega^{2}}{\left(m n \pi^{2}\right)\left[\left(\frac{n \pi}{L}\right)^{2}+\left(\frac{m \pi}{L}\right)^{2}\right]} \sin \left(\frac{m \pi x^{*}}{L}\right) \sin \left(\frac{n \pi y^{*}}{L}\right)
$$

with $m, n \in \mathbb{N}_{o d d}, x^{*}=x+\frac{L}{2}, y^{*}=y+\frac{L}{2}$. The flow is then characterized by a zero free surface pressure and a negative inner pressure. We confront here the results given by standard SPH, RSALE (MUSCL HLLC Sweby) and $\gamma-S P H-A L E$. We set $L=1 \mathrm{~m}, \omega=5 \mathrm{rad} / \mathrm{s}, h=2.0 \Delta x, \alpha=0.04, \gamma=1.0$ with $\Delta x=5 \mathrm{~mm}$ (initial spacing giving a resolution of 40000 particles), $\rho_{0}=1000 \mathrm{~kg} / \mathrm{m}^{3}$ and we use Wendland kernel functions. We also set $c_{0}=10 v_{\max }=35 \mathrm{~m} . \mathrm{s}^{-1}$, corresponding to a weakly-compressible formulation (Mach number $M=0.1$ ). The structural stability of each schemes is evaluated thanks to a comparison with a FE solution. The FE mesh is then fine enough to limit the element distortions and stay in agreements with the reference solution up to $0.4 \mathrm{~s}[51]$.

Regarding $\gamma-S P H-A L E$ stability, as introduced in section 6.1 and 6.2 , the stability limit $\alpha=\gamma=0.7(42)$ still is too dissipative and is not optimal. Indeed, the huge amount of artificial viscosity drastically decreases the Kinetic Energy prematurely stopping the rotation. The experimental stability study provides the following referential stability intervals

$$
\left\{\begin{array}{l}
N_{C F L}=0.9 N_{0.3} \\
\gamma_{\text {exp }} \in[0.002 ; 2.3] \\
\alpha_{\text {exp }} \in[0.01 ; 1.2]
\end{array}\right.
$$

According to section 6.1 remarks we can then deduce that

$$
\left\{\begin{array} { c } 
{ f _ { \gamma } ^ { - } = \frac { 0 . 0 0 2 } { N _ { C F L } } } \\
{ f _ { \gamma } ^ { + } = 2 . 3 N _ { C F L } }
\end{array} \quad \text { and } \left\{\begin{array}{c}
f_{\alpha}^{-}=\frac{0.01}{N_{C F L}} \\
f_{\alpha}^{+}=1.2 N_{C F L}
\end{array}\right.\right.
$$

and, as exposed in Table 2, we can predict the stability intervals in function of the CFL $N$.

Figure 5 top row shows the pressure fields obtained by standard SPH. It shows that nonphysical oscillations appear at time $t=0.12 \mathrm{~s}$ ( $5 \mathrm{left}$ ). At $t=0.24 \mathrm{~s}$ ( 5 center) tensile instability appears increasing then the pressure evaluation errors by creating cavities. This phenomenon worsen in time and generates the fragmentation of the domain depicted at $t=0.4 s$ ( 5 right). In purely Lagrangian $\left(v_{0}=v\right)$ both RSALE and $\gamma-S P H-A L E$ behave similarly to standard SPH and suffer from tensile instability generating the domain fragmentation. To prevent this phenomenon, we use the PST introduced in section 4.3. RSALE results are depicted on figure 5 middle 


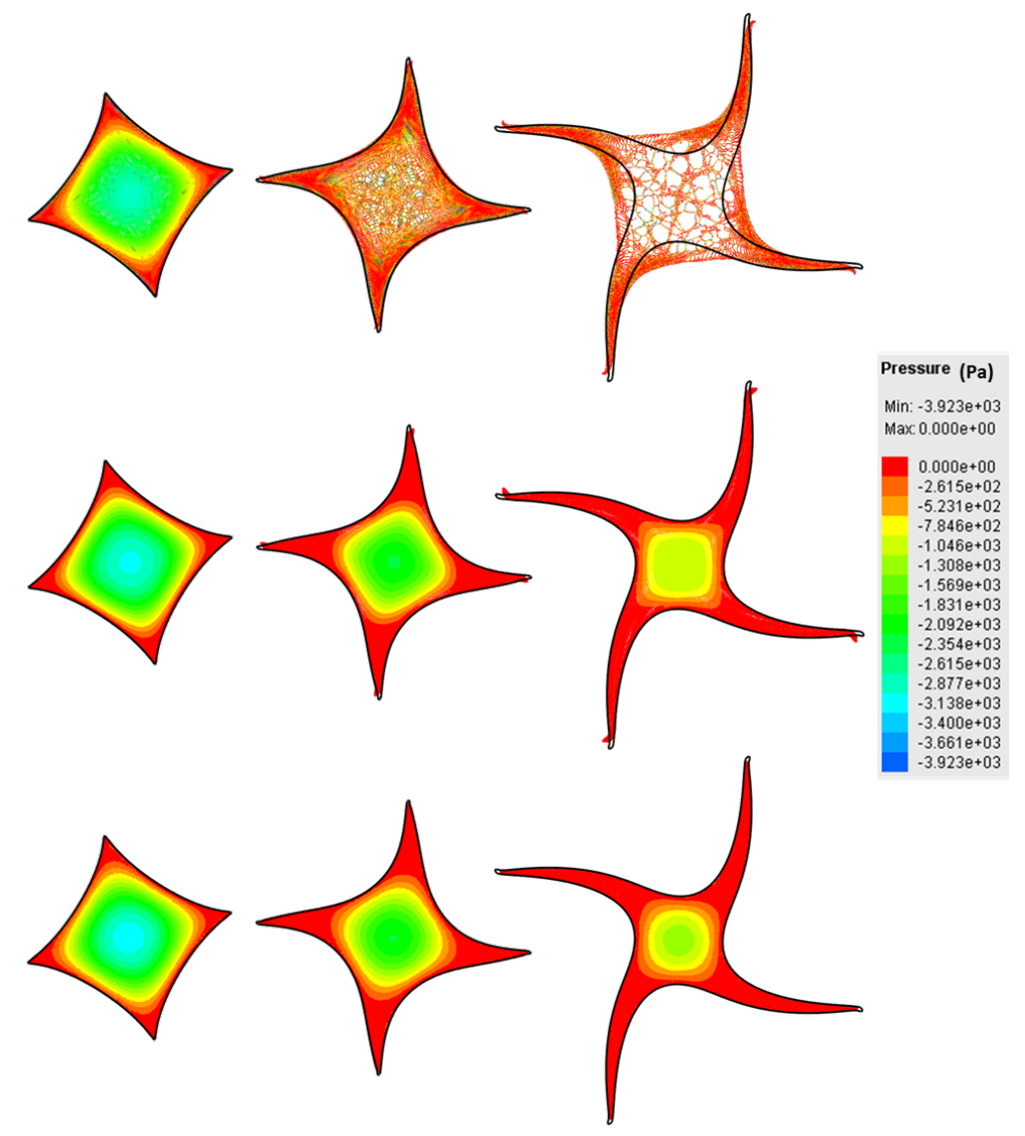

Figure 5: Rotating Square Pressure field (Pa) with: 1- standard SPH (top), 2-RSALE with PST (middle), 3- $\gamma-S P H-A L E$ with PST (bottom) and4-FE solution (black solid line) at: $\boldsymbol{a}$ ) $t=0.12 \mathrm{~s}$ (left), $\boldsymbol{b}$ ) $t=0.24 \mathrm{~s}$ (center) and $\boldsymbol{c}) t=0.4 s$ (right) 


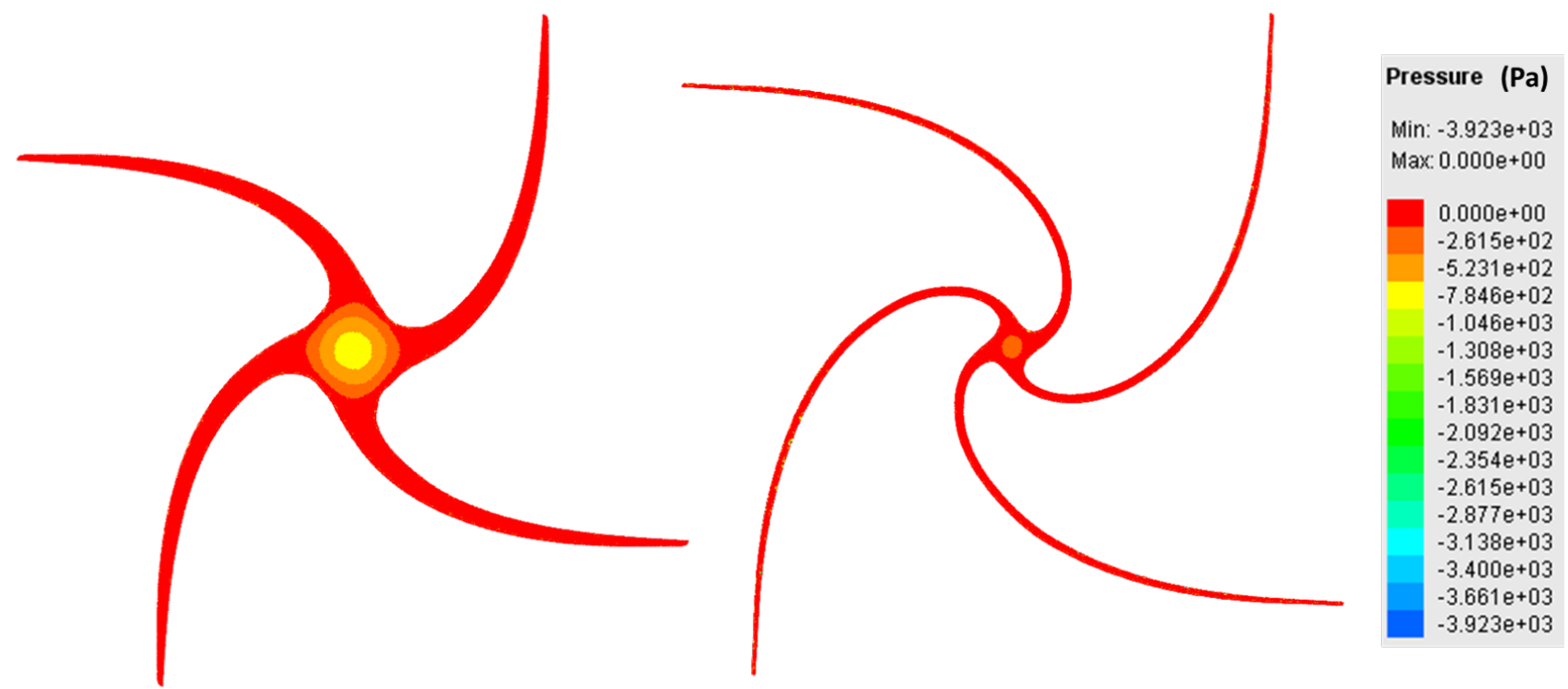

Figure 6: Rotating Square $\gamma-S P H-A L E$ Pressure field (Pa) at: $\boldsymbol{a}) t=0.6 s$ (left) and $\boldsymbol{b}$ ) $t=1.0 s$ (right)

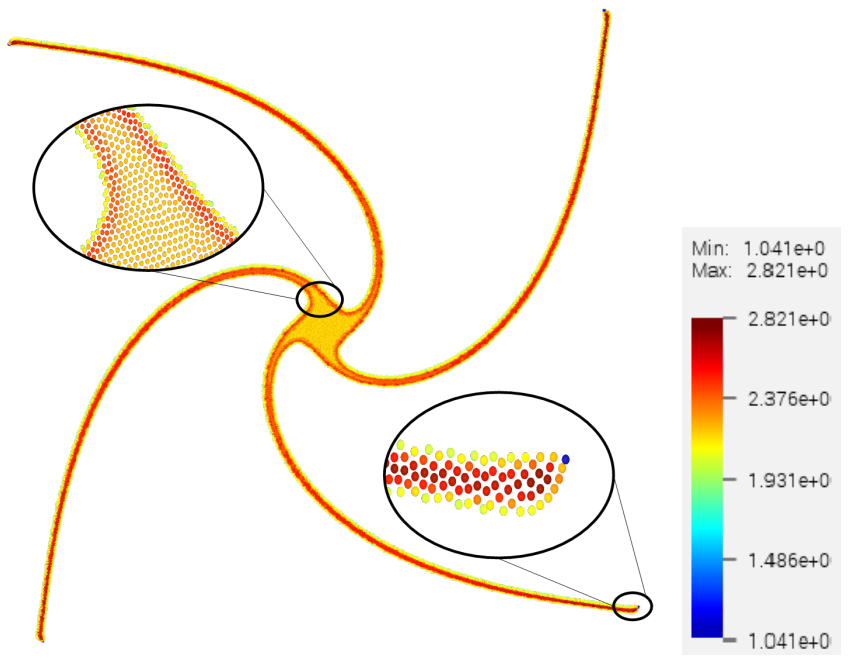

Figure 7: Rotating Square $\gamma-S P H-A L E$ distribution of the geometrical constant $\mathrm{C}$ at $t=1.0 \mathrm{~s}$

row. We can see that there are no spurious pressure oscillations or tensile instability up to $t=0.4 s$ (right). A slight rounded effect appears at the end of the four jets as only deviation with the FE solution. $\gamma-S P H-A L E$ results are exposed on figure 5 bottom row. They do not show any spurious oscillations, tensile instability or rounded effect up to $t=0.4 s$ (right). Regarding this late aspect, the calibration of the viscous parameter $\alpha$ reveals that the jets are particularly sensitive to the amount of artificial viscosity introduced in the scheme. Adjusting $\alpha$ allows then to efficiently prevent the rounded effect in $\gamma-S P H-A L E$. On the contrary with RSALE the viscosity is intrinsic and cannot be easily controlled. $\gamma-S P H-A L E$ results (5 bottom row) show no deviation with the FE solution (solid line). For both RSALE and $\gamma-S P H-A L E$, PST allows an efficient particle reorganization preventing any tensile instability. No fragmentation of the domain appears ensuring then the structural stability. Thus, while enough particles are available to reorganize the particle distribution (meaning that particles are not too far from each other) the structural stability is insured. Regarding the computation times RSALE calculations are 1.17 times longer than $\gamma-S P H-A L E$ ones as expected due to RSALE complexity. Figure 6 depicts the results provided by $\gamma-S P H-A L E$ up to 1 s. Regarding the constant $C$ introduced in section 3.1, as announced we can see on figure 7 that $C$ stays bounded. Note that the maximum value is achieved in particle rarefaction areas (in the near free surface area and within the jets).

Figure 8 displays the time variation of pressure extracted at the center of the patch for RSALE with PST and $\gamma-S P H-A L E$ with PST for $\Delta x=3 \mathrm{~mm}$. The results are confronted to the incompressible solution 


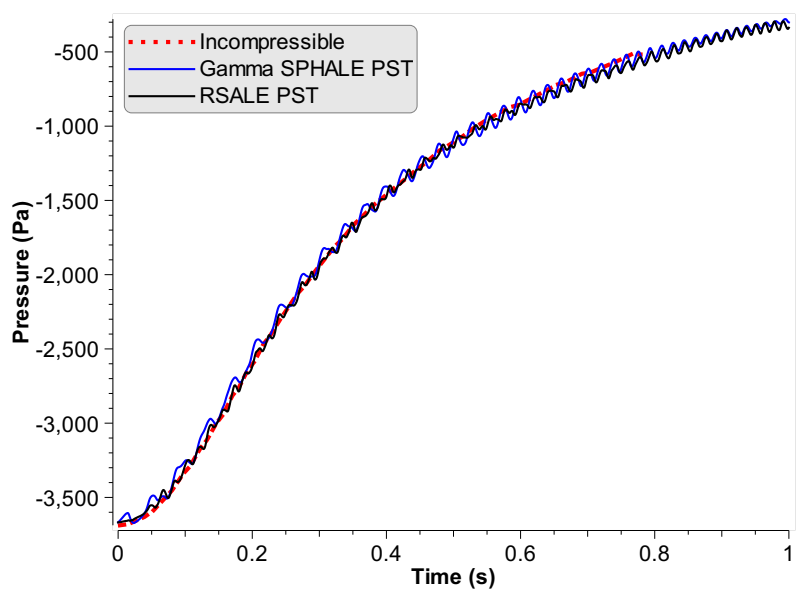

Figure 8: Rotating Square Pressure at the patch center RSALE PST (black), $\gamma-S P H-A L E$ PST $\gamma=1$ (blue) and Incompressible solution (red dotted line)

achieved by Boundary Element Mehtod (BEM) extracted from [12]. For both scheme Figure 8 shows pressure curves oscillating around the incompressible solution. We recall here that we work in a weakly-compressible context. Such hypothesis consists in allowing slight compressibility effects in the computation to approximate an incompressible flow. In practice the sound speed is set as $c_{0}=10 v_{\max }$ where $v_{\max }$ is the maximum flow velocity (Mach number $M=0.1$ ). Thus, as such artificial sound speed is lower than the real one, the computation time step is increased, and the computation is accelerated. Regarding the flow properties, for compressible flows the relative density variation is of the order of $M^{2}[6]$. Then, working in a weakly-compressible framework means that a $1 \%$ relative variation is allowed on the flow density. In our case, working with a linear barotropic EOS, such density variation is directly converted to pressure variations. Thus the pressure oscillations depicted on figure 8 are in fact representative of the weakly-compressible assumption. They correspond to the physical acoustic part of the flow. The incompressible solution can then be extracted thanks to a filtering strategy ([51]) but does not corresponds to the converged solution.

Figures 9-left depicts the pressure extracted at the center of the patch for $\gamma-S P H-A L E$ PST for three particle refinements: $\Delta x=3 \mathrm{~mm}$ (green), $\Delta x=10 \mathrm{~mm}$ (red) and $\Delta x=20 \mathrm{~mm}$ (purple). We can see the more the particle number is increased, the smaller the oscillation amplitude is. The scheme converges through the oscillating weakly-compressible solution. Note that the curves oscillate at the same frequency and in particular the first oscillation has a frequency $f_{\exp }=25.9 \mathrm{~s}^{-1}$. It corresponds to the theoretical fundamental acoustic frequency of a squared membrane of length $\mathrm{L}$ (theoretically $f_{t h}=\frac{c_{0}}{\sqrt{2} L}=25 \mathrm{~s}^{-1}$ ). The acoustic part of the flow is then correctly captured. Figure 9-right shows the error in $L_{2}$ norm on the pressure at the center of the patch for $\gamma-S P H-A L E$ with PST for $\gamma \in\{0.3 ; 1.0 ; 2.3\}$. The error is evaluated as

$$
\epsilon_{L_{2}}=\frac{\left(\Sigma_{n}\left|p_{r e f}^{n}-p_{e x p}^{n}\right|^{2}\right)^{\frac{1}{2}}}{\left(\Sigma_{n} p_{r e f}^{n}\right)^{\frac{1}{2}}}
$$

where $p_{\text {ref }}$ corresponds to the pressure of the converged case. Regarding the results we take the case $\Delta x=$ $3 m m, \gamma=0.3$ as the reference. The results are presented in Table 3. First, we can see that the convergence previously introduced is confirmed (convergence order between 0.682 and 1.218). Second, increasing $\gamma$ tends to decrease the order of convergence and also increases the error (between $0.5 \%$ and $11 \%$ ). It appears on Figure 10 which displays the effects of $\gamma$ on the pressure at the patch center. We take $\Delta x=5 \mathrm{~mm}$ giving 40000 particles. As introduce in the shock tube case, here also increasing $\gamma$ directly impacts the pressure oscillations by damping their amplitude more efficiently (for late stages Figure 10-right). However, increasing $\gamma$ also increases the oscillation amplitude for early stages (Figure 10-left) explaining why the error in Table 3 increases with $\gamma$. This amplitude is even more amplified that the number of particles is low.

Figure 11 depicts the results given by RSALE with PST (top row) and $\gamma-S P H-A L E$ with PST (bottom row) at a Mach number $M=0.01$ (meaning that $c_{0}=100 v_{\max }$ ) and $\Delta x=10 \mathrm{~mm}$ (around 10000 particles). RSALE results (top row) show a noisy pressure field (with a sudden drop on the free surface) and a severe deviation with the FE solution. On the contrary, $\gamma-S P H-A L E$ (bottom row) provides results as convincing as before (figure 5). In case of RSALE, as introduced in section 1.2, decreasing $M$ generates intense pressure 

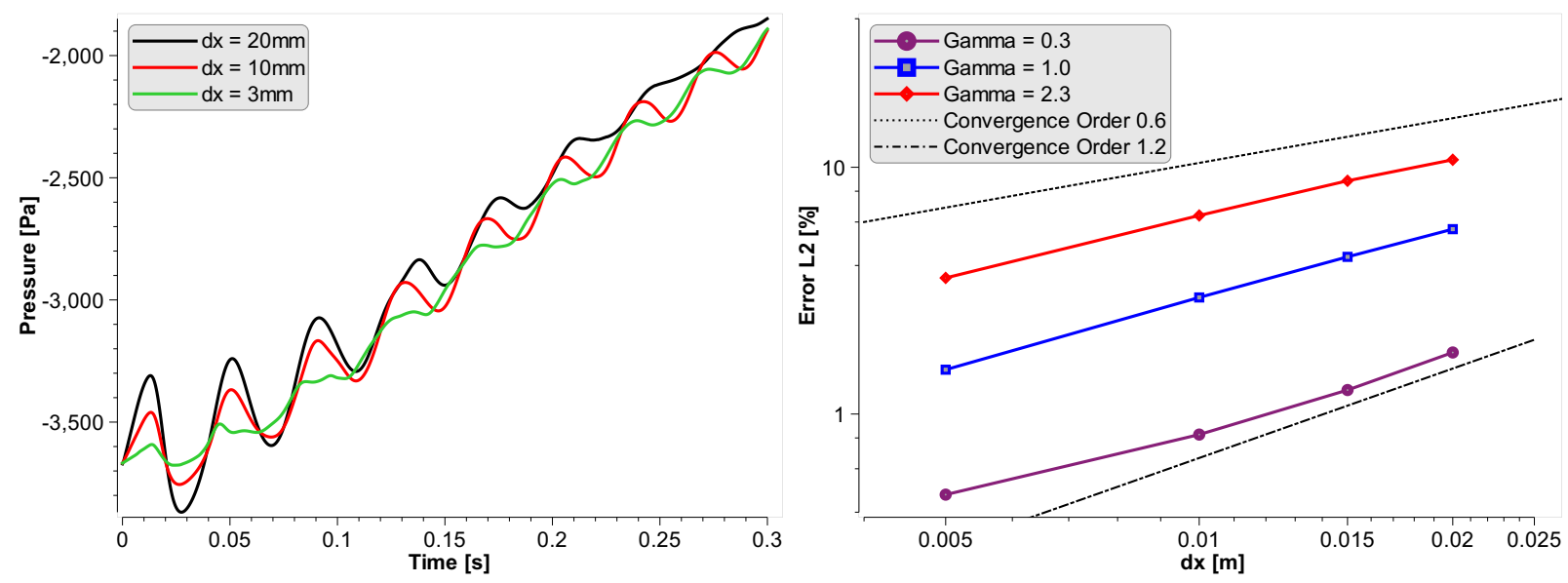

Figure 9: Rotating Square Convergence study on the Pressure at the patch center for $\gamma-S P H-A L E$ PST. Left plot: $\gamma=1, \alpha=0.04$ for three particle refinements: $\Delta x=3 \mathrm{~mm}$ (green), $\Delta x=10 \mathrm{~mm}$ (red) and $\Delta x=20 \mathrm{~mm}$ (black); Right plot: Error in L2 norm (50) for $\gamma \in\{0.3 ; 1.0 ; 2.3\}$

\begin{tabular}{|c|c|c|c|c|c|}
\cline { 2 - 6 } \multicolumn{1}{c|}{} & $\Delta x(\mathrm{~mm})$ & 20 & 15 & 10 & 5 \\
\hline \hline \multirow{2}{*}{$\gamma=0.3$} & $\epsilon_{L_{2}}(\%)$ & 1,776 & 1,251 & 0,826 & 0,471 \\
\cline { 2 - 6 } & Order & & 1,218 & 1,025 & 0,809 \\
\hline \hline \multirow{2}{*}{$\gamma=1.0$} & $\epsilon_{L_{2}}(\%)$ & 5,613 & 4,335 & 2,968 & 1,513 \\
\cline { 2 - 6 } & Order & & 0,898 & 0,934 & 0,972 \\
\hline \hline \multirow{2}{*}{$\gamma=2.3$} & $\epsilon_{L_{2}}(\%)$ & 10,727 & 8,816 & 6,377 & 3,561 \\
\cline { 2 - 6 } & Order & & 0,682 & 0,799 & 0,841 \\
\hline
\end{tabular}

Table 3: Rotating Square $\gamma-S P H-A L E$ convergence in $L_{2}$ norm on the pressure extracted at the center of the patch for $\gamma \in\{0.3 ; 1.0 ; 2.3\}$
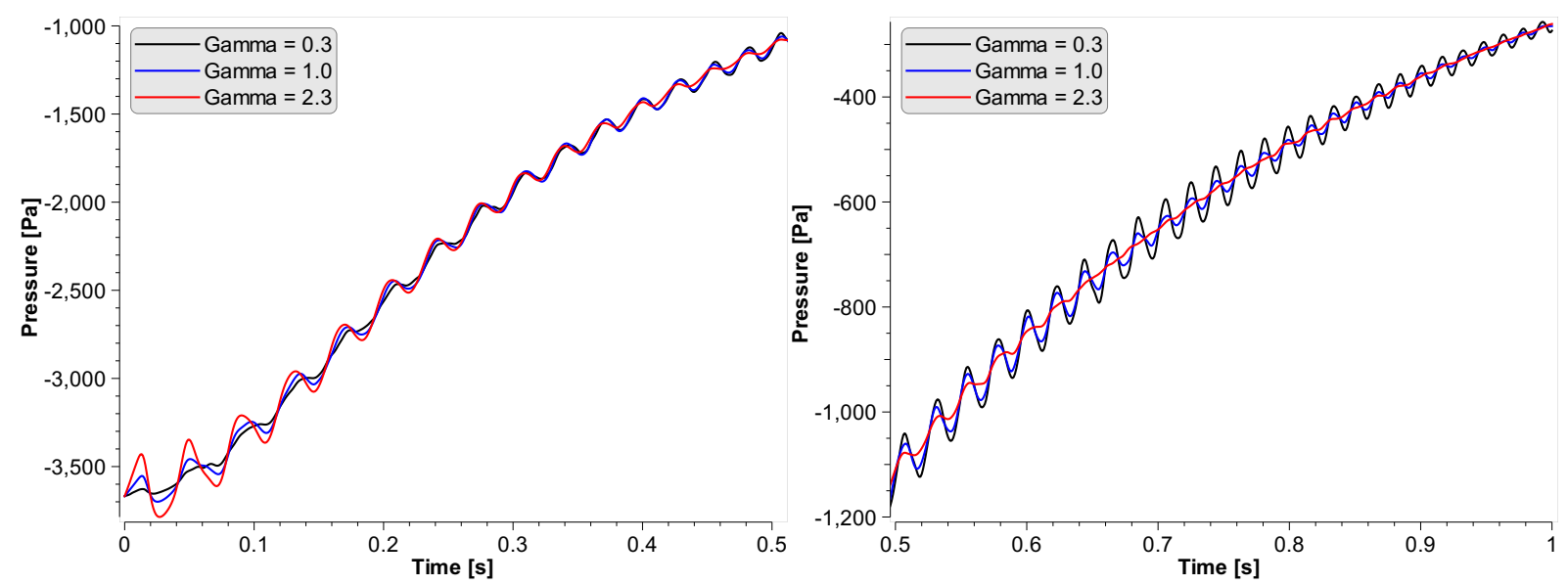

Figure 10: Rotating Square Pressure at the patch center for $\gamma-S P H-A L E$ PST with $\gamma \in\{0.3 ; 1.0 ; 2.3\}$ (left) early stages, (right) late stages 


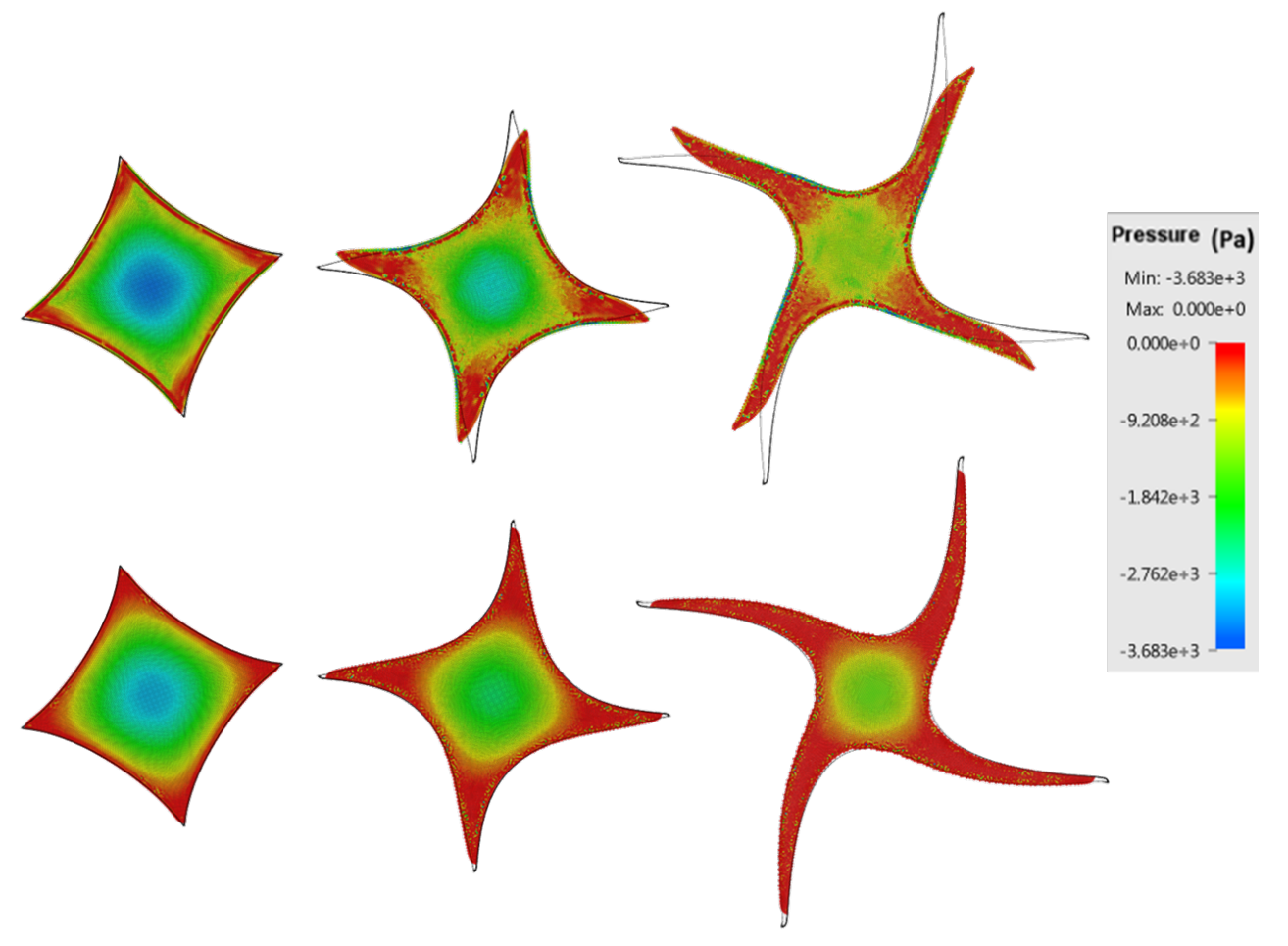

Figure 11: Rotating Square Pressure fields $(\mathrm{Pa})$ at $M=0.01$ with: 1- RSALE with PST (top), 2- $S P H-A L E$ with PST (bottom) and $3-\mathrm{FE}$ solution (black solid line) at: $\boldsymbol{a}) t=0.12 \mathrm{~s}$ (left), $\boldsymbol{b}) t=0.24 \mathrm{~s}$ (center) and $\boldsymbol{c}) t=0.4 \mathrm{~s}$ (right)

fluctuations (amplification factor of $1 / M$ ) corrupting the whole pressure field. The scheme remains stable in the weakly-compressible limit, but not if the Mach number drops. Such instabilities are prevented in $\gamma-S P H-A L E$ thanks to the stabilizing low-Mach scheme. In case of $\gamma-S P H-A L E$, according to $(29,31) \alpha_{i j}=\alpha \bar{c}_{i j} \bar{\rho}_{i j}\left\|\boldsymbol{A}_{i j}\right\|$ and $\gamma_{i j}=\frac{\gamma}{2 \tilde{c}_{i j} \tilde{\rho}_{i j}}$. Then increasing $c_{0}$ also increases the amount of artificial viscosity introduced in the scheme and decreases the low-Mach stabilization (in practice $\bar{c}_{i j}=\tilde{c}_{i j}=c_{0}$ ). However, we can see on figure 11 bottom plot that a satisfying behavior can be recovered with $\gamma-S P H-A L E$. Such results are achieved with $\alpha=0.004$ and $\gamma=2.0$. To limit the diffusive behavior, the classical strategy is to increase the number of particles and then the computation times. But in case of $\gamma-S P H-A L E$ we can keep the same number of particles and just tune $\alpha$ and $\gamma$ to provide the expected solution. Figure 12 displays the pressure at the center of the patch for $\gamma-S P H-A L E$ (same configuration as figure 11) and RSALE. In case of RSALE, we can see a low frequency oscillation, characteristic of the low-mach behavior ruling the pressure fields, for $\Delta x=10 \mathrm{~mm}$ and $\Delta x=5 \mathrm{~mm}$. We must decrease $\Delta x$ down to $3 \mathrm{~mm}$ (around $110000 \mathrm{SPH}$ ) to recover a correct pressure field. Also, note that there is still a deviation with the FE solution and a severe rounded effect at the end of the four jets (late stages). On the contrary, $\gamma-S P H-A L E$ preserves both pressure field and structural stability with only $\Delta x=10 \mathrm{~mm}$ (40000 SPH). RSALE calculation is then 33 times longer than $\gamma-S P H-A L E$.

$\gamma-S P H-A L E$ and RSALE are able to provide reliable results in a weakly compressible framework. A stable and consistent combination with regularizing techniques as PST is allowed by the ALE formalism. It efficiently prevents tensile instability and increases the accuracy. However, in case of $\gamma-S P H-A L E$, a direct control on the artificial viscosity as well as the low-mach scheme allows to improve the accuracy. It is achieved by limiting the viscous dissipation and by efficiently damping the spurious pressure oscillations in reduced computation times with respect to RSALE. These abilities are even more effective and crucial below the weakly compressible limit, where RSALE suffers from strong instabilities due to its low-Mach limitations.

\subsection{Oscillating Drop}

Another challenging benchmark test is the 2D oscillating drop under a central conservative force field. Originally proposed by Monaghan \& Rafiee [6], it has been widely studied as a relevant test in terms of particle regularization techniques and energy conservation. See [32, 31, 52]. It is based on a $2 \mathrm{D}$ circular patch of fluid 


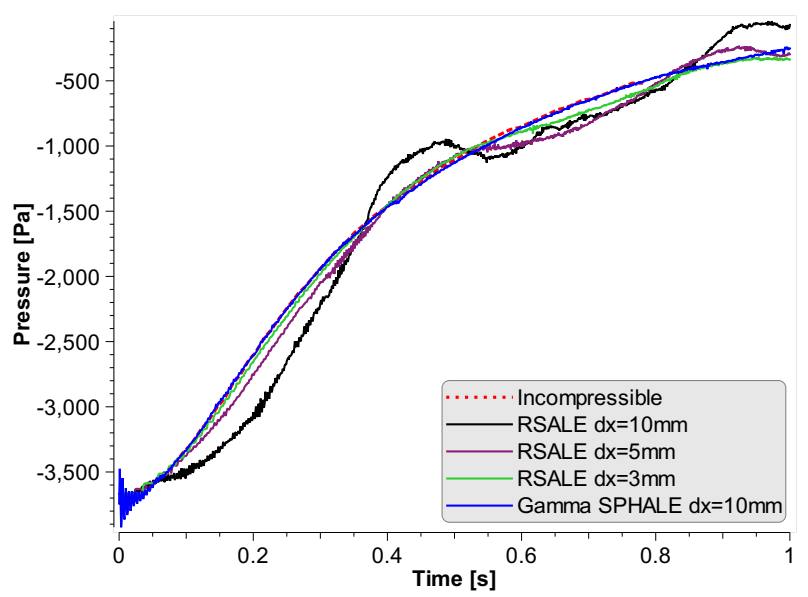

Figure 12: Rotating Square Pressure at the center of the patch for RSALE PST and $\gamma-S P H-A L E$ PST at $M=0.01$

free from any boundary condition and only evolving under the action of a central force field $\boldsymbol{f}=-A_{0}^{2} \boldsymbol{r} . \boldsymbol{r}$ defines the particle position and $A_{0}$ the velocity amplitude. The numerical domain $\Omega$ is a patch of radius $R$, centered at the origin and subjected to the initial velocity field set as

$$
\left\{\begin{array}{l}
v_{x}=-A_{0} x \\
v_{y}=A_{0} y
\end{array}\right.
$$

The flow is characterized by a zero free surface pressure and a positive inner pressure. We set $R=1 \mathrm{~m}$, $A_{0}=100 \mathrm{~s}^{-1}, h=1.6 \Delta x, \alpha=0.005, \gamma=0.7, \rho_{0}=1000 \mathrm{~kg} / \mathrm{m}^{3}$ and we use Cubic Spline kernel functions. We also set $c_{0}=15 A_{0} R=1500 \mathrm{~m} . \mathrm{s}^{-1}$ and the shifting magnitude is set to 0.1 . The structural stability is evaluated thanks to a comparison with the analytical solution available in the literature [6]. The standard SPH scheme is characterized by a noisy pressure field as well as an irregular particle distribution. Figure 13 depicts the patch evolution as well as the pressure field in comparison with the analytical solution in time. Figure 13-top plot shows $\gamma-S P H-A L E$ results in purely lagrangian and bottom row $\gamma-S P H-A L E$ with PST for $\frac{R}{\Delta x}=80$. In purely lagrangian we can see that the evolution of the free surface is in good agreements with the analytical solution. We can also see that during the first oscillation, particle align themselves as depicted on figure 14-left. This phenomenon is generated by the cartesian initial distribution. Under the initial velocity field, particles on the same initial line move by staying on this line. At time $t=36 \mathrm{~ms}$ under the effect of numerical errors a slight disorder is introduced in the particle distribution allowing to break the lines and to stay in agreement with the analytical solution (self-reorganization process [34]). See the case Stretching of an initially circular patch of fluid proposed by Monaghan [6] for a similar behavior. To prevent this phenomenon a slight disorder is generally introduced in the initial particle distribution. Also, a smooth pressure field is preserved thanks to the low mach scheme. In case of $\gamma-S P H-A L E$ with PST (figure 13 bottom), we can see that the evolution of the free surface is also in very good agreement with the analytical solution. It preserves a smooth pressure field as well. However, thanks to PST the particle distribution stays regular during the whole computation and does not need any initial disorder (figure 14-right).

Figure 15 shows the time variation of the horizontal semi-axis for $\gamma-S P H-A L E$ in purely lagrangian (purple) and with PST (blue, red) compared to the analytical solution (black). As introduced above, cases with $\frac{R}{\Delta x}=80$ are both in good agreements with the analytical solution but, the error still increases in time. As the scheme is dissipative, it loses energy over time and the drop oscillation amplitude decreases then in time. However, we can see with the case $\gamma-S P H-A L E$ PST $\frac{R}{\Delta x}=200$ (red) that the energy loss decreases with the increase of particles number. $\gamma-S P H-A L E$ converges in space through the analytical solution.

Contrary to the rotating square patch of fluid case (previous section), the pressure field is not initialized to the incompressible pressure, meaning that $p_{0}=0$. Hence the oscillation magnitude on Figure 16. Figure 16 displays the pressure time variation extracted at the center of the patch for different values of $\gamma$. The theoretical initial acoustic frequency is given by $f_{t h}=0.7655 \frac{c_{0}}{2 R}=574.12 \mathrm{~s}^{-1}$ and is recovered in each case. In addition, we can see that increasing $\gamma$ allows to efficiently damp the pressure oscillations and limits their amplitudes. However, it means loosing the acoustic part of the flow.

Figure 17 displays the mechanical energy loss for $\gamma-S P H-A L E$ PST with $\frac{R}{\Delta x}=50$ (green), $\frac{R}{\Delta x}=100$ (blue) and $\frac{R}{\Delta x}=200$ (red) compared to $\gamma-S P H-A L E$ Lagrangian with $\frac{R}{\Delta x}=200$ (black). We have then $E m=E_{k}+E_{p}$ where $E_{k}$ and $E_{p}$ are respectively the kinetic and potential energies. We can see that as expected 

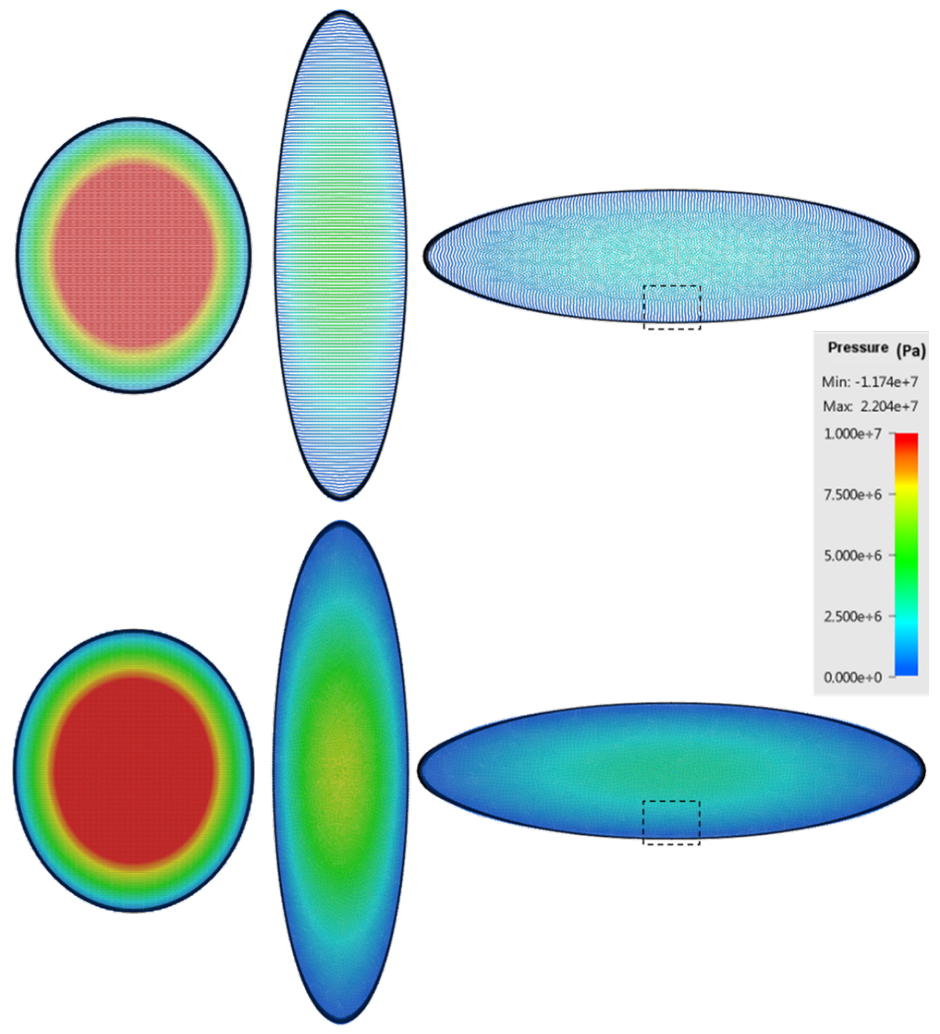

Figure 13: Oscillating Drop Pressure field $(\mathrm{Pa})$ achieved by $\gamma-S P H-A L E$ in purely lagrangian (top) and with PST (bottom) at $t=0.8 \mathrm{~ms}$ (left), $t=11.2 \mathrm{~ms}$ (center) and $t=36 \mathrm{~ms}$ (right) for $\frac{R}{\Delta x}=80$, compared with the analytical solution (black solid line)

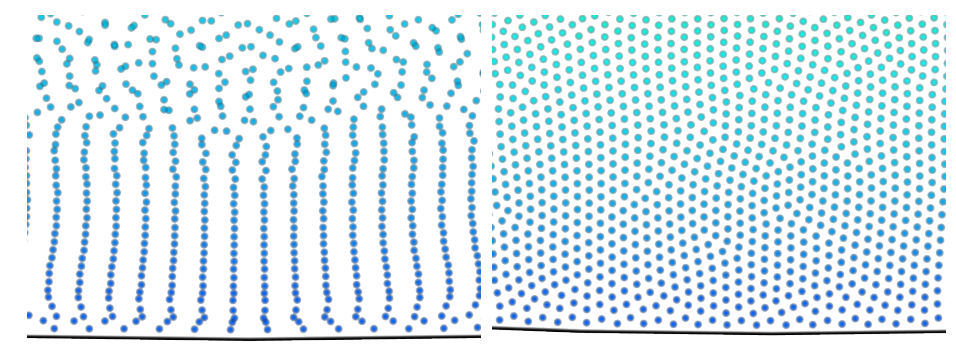

Figure 14: Oscillating Drop Zoom on the particle distributions for $\gamma-S P H-A L E$ in purely lagrangian (left) and with PST (right) at $t=36 \mathrm{~ms}$

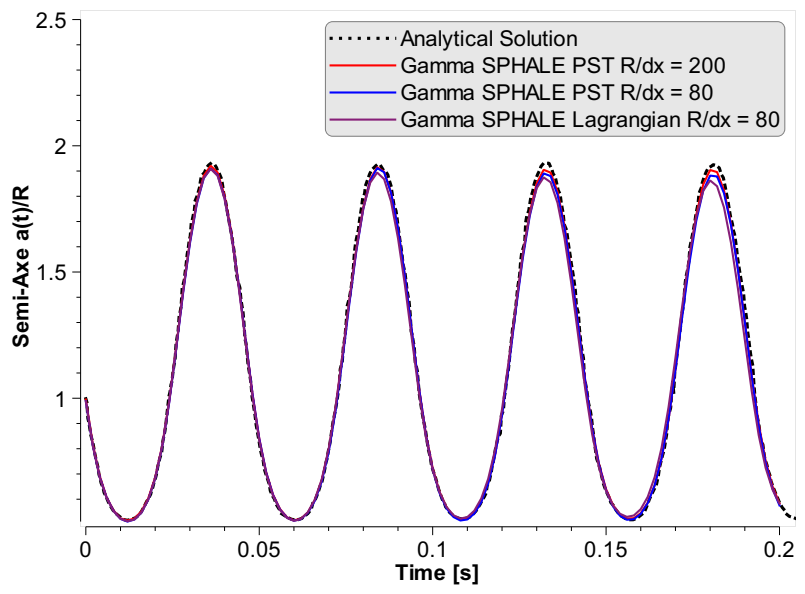

Figure 15: Oscillating Drop Time variation of the horizontal semi-axis $a$ for $\gamma-S P H-A L E$ PST with three particle refinements 


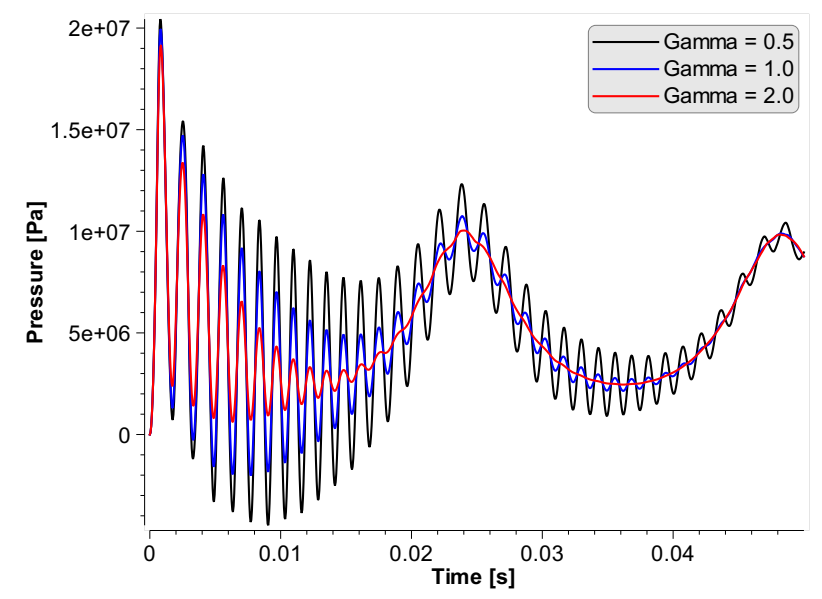

Figure 16: Oscillating Drop Pressure at the center of the patch for $\gamma-S P H-A L E$ with PST for $\gamma \in$ $\{0.5 ; 1.0 ; 2.0\}$

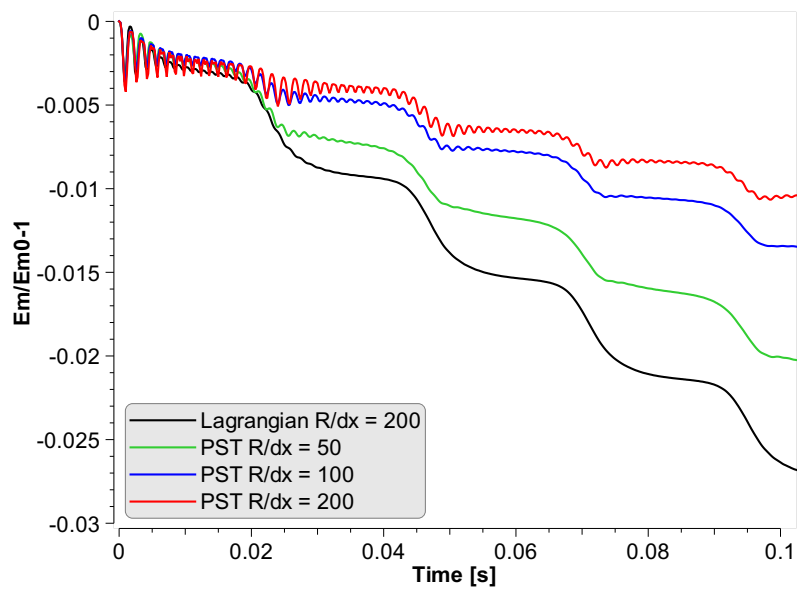

Figure 17: Oscillating Drop Evolution of the mechanical energy loss of $\gamma-S P H-A L E$ for three spatial resolutions $\Delta x$

for each case the mechanical energy loss increases over time. As introduce before with Figure 15, this loss corresponds to the energy dissipated by the artificial viscosity and the low-Mach scheme. In particular, we can see that PST is crucial regarding the energy loss. Indeed, using PST (Figure 17 red) provides a more accurate solution than in Lagrangian (Figure 17 black) as the particle distribution regularity is preserved during the whole computation. Besides, the mechanical energy loss decreases with the increase of particle number. The scheme consistency is then confirmed. Note that, as increasing the particle number decreases the dissipation, and particularly the one linked to $\gamma$, the acoustic part of the flow also appears in the energy measurements (oscillations on Figure 17). Also, the total energy is preserved (relatively to the other energetic quantities).

$\gamma-S P H-A L E$ is able to provide reliable results in a weakly compressible framework in the presence of external forces. Here again, the consistent combination with PST and the low-mach scheme efficiently insures a smooth pressure field as well as a regular particle distribution. The total energy is also preserved thanks to an efficient control on the artificial diffusion through the scheme parameters.

\subsection{Dam Break}

The last benchmark test is the case of a 2D Dam-break flow. It is a classical test in the SPH framework as it combines complex situations as free surfaces, boundary conditions, violent impact and free surface reconnections. See $[33,28,30]$ in the context of PST. The numerical model is described in [47]. It consists in a fluid column of height $H=600 \mathrm{~mm}$, and length $l=2 \mathrm{H}$ placed in the corner of a tank of length $L=5.366 \mathrm{H}$. We set $\rho_{0}=1000 \mathrm{~kg} / \mathrm{m}^{3}$ and $c_{0}=80 \mathrm{~m} \cdot \mathrm{s}^{-1}$. The column is initially at rest and is subjected to gravity at $t=0 \mathrm{~s}$. Under the gravity effect, the column collides and flows on the bottom of the tank following a contact boundary condition. The flow front impacts then the tank wall generating a breaking wave. This wave breaks back on the 


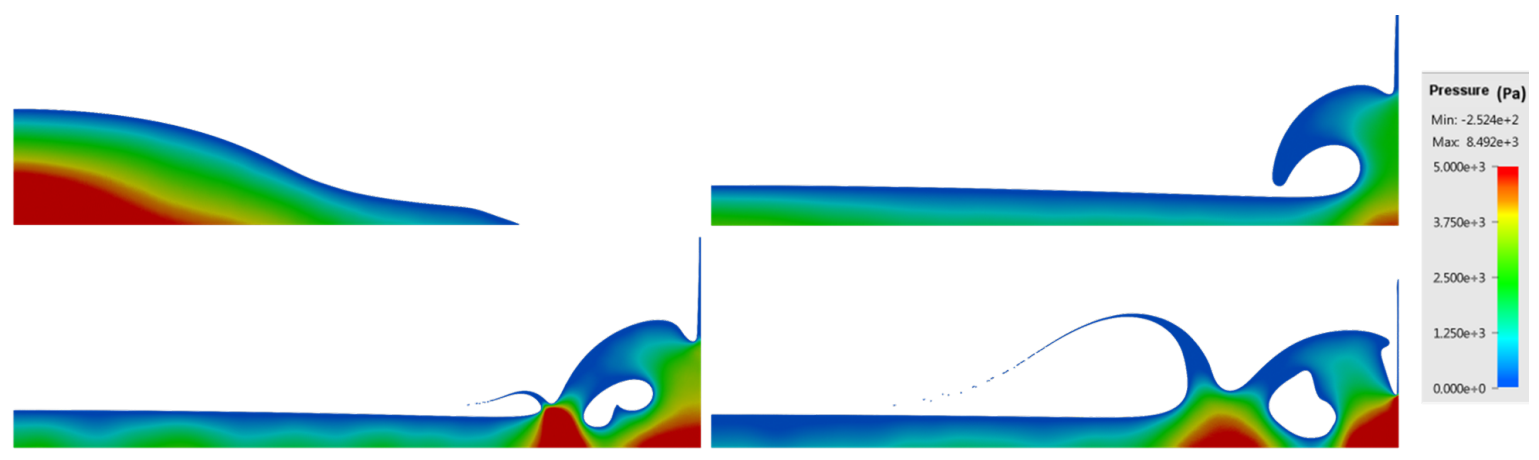

Figure 18: Dam Break Pressure field with $\gamma-S P H-A L E$ with PST and $\Delta x=1 \mathrm{~mm}$ at $t=0.4 s$ (top left), $t=1.48 \mathrm{~s}$ (top right), $t=1.56 \mathrm{~s}$ (bottom left) and $t=1.72 \mathrm{~s}$ (bottom right)

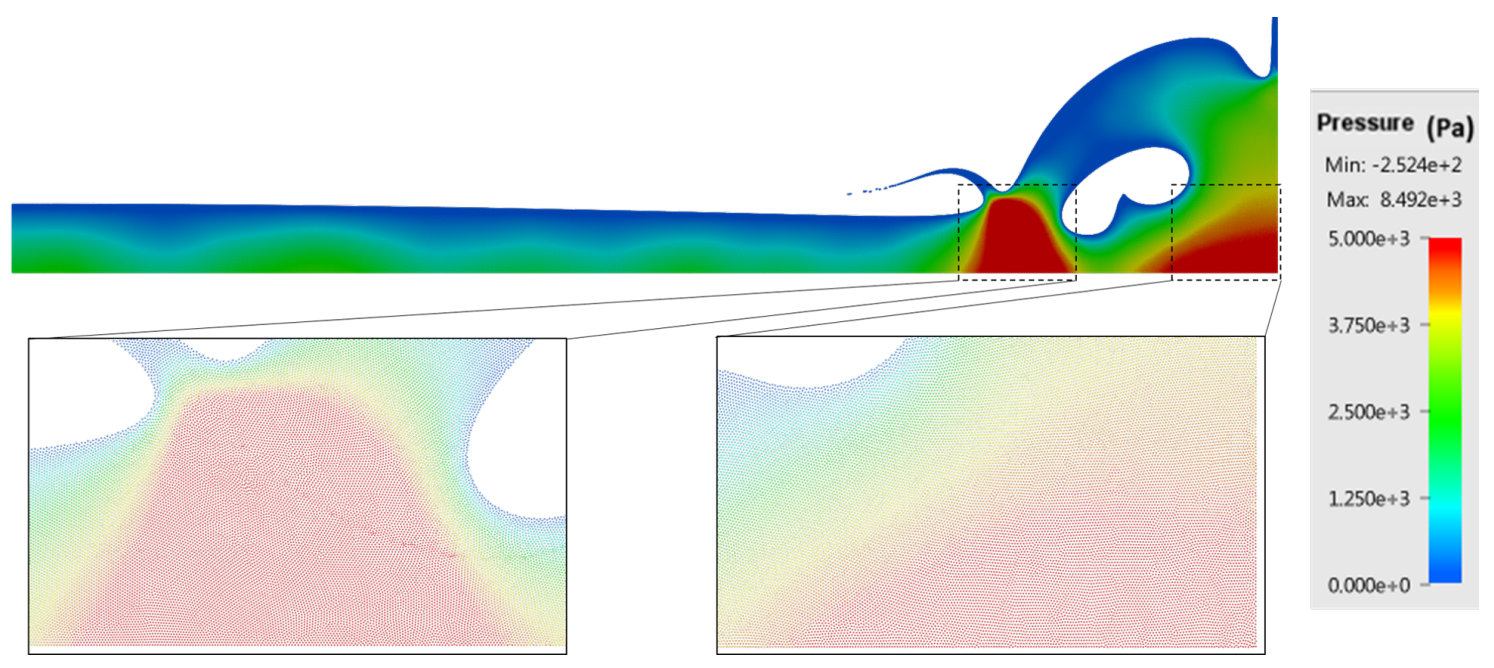

Figure 19: Dam Break Particle Distribution at first and second impacts at $t=1.56 \mathrm{~s}$ for $\Delta x=2 \mathrm{~mm}$

top of the flow leading to free surface reconnection. The flow fills then the whole tank and return to equilibrium. Dynamic ghost particles are used to model the contact boundary condition on the wall. In standard SPH the global kinematic of the flow is capture but it provides a noisy pressure field and suffers from particle clumping. Figure 18 displays the results provided by $\gamma-S P H-A L E$ PST with $\alpha=0.03, \gamma=1.0$ and $\Delta x=1 \mathrm{~mm}$ (generating $718201 \mathrm{SPH}$ ). The flow against the bottom of the tank, the breaking wave generated by the impact on the wall and the free surface reconnection are correctly captured by preserving as well, a smooth pressure field. Thanks to PST, the particle distribution also stays regular during the whole computation. As appearing on figure 19 such regularity is achieved in the corner impact (figure 19 right) and on the reconnection area as well (figure 19 left).

A pressure probe $P 0$ is placed on the impacted wall (height $120 \mathrm{~mm}$ ) to extract the pressure and compare it to the experimental one. Figure 20 left shows the pressure fields at $P 0$ for different spatial resolutions at $\gamma=1.0$ and the experimental result. We can see that the global behavior is recovered in each case. A pressure impulse appears at the impact against the wall at $t=0.6 \mathrm{~s}$. The pressure stays on a plateau up to $t=1.4 \mathrm{~s}$ when a second impulse appears. It corresponds to a new impact generated by the breaking wave. Up to this second impact, increase the spatial resolution allows to capture more accurately the first pressure impulse. It also decreases the pressure noise. After $1.4 s$, the higher the spatial resolution is, the more delayed is the second impulse and a second plateau is reach for the three higher resolutions. Also, whereas for the three lower resolution the pressure stays positive similarly to the experiment, for the last two resolutions the pressure goes to zero. The first plateau is generated by the presence of fluid against the probe during the whole breaking process. The breaking generates the second impulse which, if the viscosity is small enough, result in a second plateau. Indeed, as increase the resolution decreases the artificial viscosity, the fluid moves easier (it stays longer against the probe). Then, under the effect of gravity the fluid goes down to the bottom of the tank. Temporary there is no fluid against the probe (resulting in zero pressure). On the contrary, for the three low resolution, the fluid motion is reduced leading to a sooner return to equilibrium. Regarding the effect of $\gamma$ we can see on 

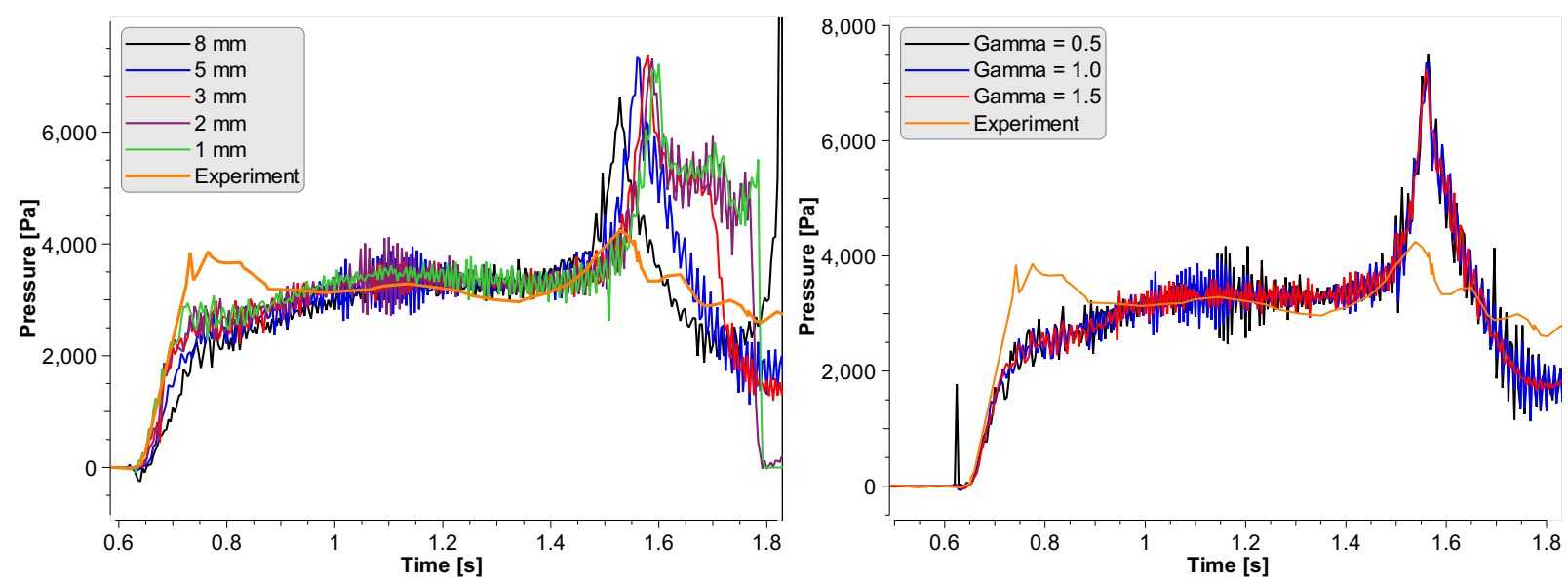

Figure 20: Dam Break Pressure on the impacted wall at position P0 achieved by $\gamma-S P H-A L E$ with PST for different $\Delta x$ (left) and $\gamma$ (right)

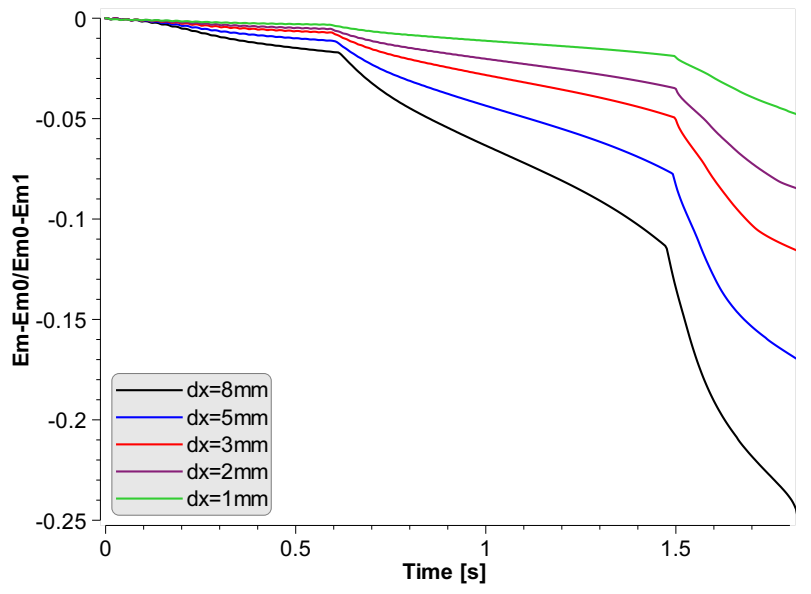

Figure 21: Dam Break Evolution of the mechanical energy loss in functions of $\Delta x$ for $\gamma-S P H-A L E$ with PST

figure 20 right $(\Delta x=5 \mathrm{~mm})$ that it allows also to reduce the numerical noise. Figure 21 displays the mechanical energy loss for $\Delta x \in[1 \mathrm{~mm}, 8 \mathrm{~mm}]$. Note that $E m 0$ corresponds to the initial mechanical energy (reduced to initial potential energy), and $E m 1$ to the final mechanical energy (reduced to final potential energy). We can see that to increase the spatial resolution allows to reduce the numerical diffusion induced by the stabilizing terms (low-Mach scheme, artificial viscosity) and then, to decrease the mechanical energy loss. The scheme consistency is again enforced. Note that the total energy is preserved as well.

$\gamma-S P H-A L E$ is able to provide reliable results in a weakly compressible framework in the presence of external forces, wall boundary conditions and violent flow conditions (impact, free surface reconnection). The consistent combination with PST and the low-mach scheme not only insures a smooth pressure field as well as a regular particle distribution (increasing the accuracy), but also provides results in very good agreements with the experiment. The total energy is again preserved thanks to an efficient control on the artificial diffusion through the scheme parameters. Particularly in this case, where the pressure results strongly depend on the amount of artificial viscosity introduced in the scheme (flow behavior during second impact).

\section{Conclusion}

We have proposed a new mesh-less scheme called $\gamma-S P H-A L E$ based on the combination of both SPH-ALE formulation [1] and FV low-Mach scheme [2, 3]. PDE are written in ALE formalism allowing for a simple treatment of the arbitrary velocity field, ruling the particle motion. A stabilizing term extracted from the FV low-Mach scheme is also introduced by means of a velocity corrective term (depending on a parameter $\gamma)$. Proportional to the pressure gradient it improves the state variables evaluation by smoothing spurious 
oscillations. An artificial viscosity (depending on a parameter $\alpha$ ) is combined with this $\gamma$ velocity to ensure the global stability of the scheme. The arbitrary property of the transport velocity field $v_{0}$ also allows to implement a PST increasing both accuracy and stability while maintaining the conservative and consistency property of the scheme.

For the first time a nonlinear stability analysis is performed on a ALE SPH scheme applied to Euler equations. CFL-like conditions and optimal values for the scheme parameters are exhibited to efficiently control the total energy and insure the scheme consistency. A strong theoretical background is then built to ensure the scheme stability. We evaluated the performances of $\gamma-S P H-A L E$ by comparing with RSALE used as a reference, analytical solutions and experimental results. Four test cases are treated: isentropic shock tube, rotating square patch of fluid, oscillating drop and dam break. For the shock tube case $\gamma-S P H-A L E$ provides a compressible solution in very good agreement with the RSALE and exact solutions. Thanks to the ALE formalism, such convincing results are achieved in Eulerian description, in Lagrangian description and with PST as well. But without the complexity induced by the resolution of Riemann problems and the use of slope limiters, reducing then the computation times. For the rotating square case, $\gamma-S P H-A L E$ maintains an accurate solution in the weakly-compressible limit and even below contrary to RSALE. Even if a stable and consistent combination with regularizing techniques as PST is allowed by the ALE formalism, RSALE suffers from strong instabilities due to its low-Mach limitations drastically increasing its computation times. Such instabilities are prevented with $\gamma-S P H-A L E$ thanks to a direct control on the viscous dissipation (artificial viscosity) and by efficiently damping the spurious pressure oscillations (low-mach scheme). For the oscillating drop case $\gamma-S P H-A L E$ preserves a reliable weakly compressible solution even in the presence of external forces. The combination with PST and the low-mach scheme insures a smooth pressure field as well as a regular particle distribution. It allows to stay in very good agreement with the analytical solution while preserving the total energy. Finally, for the Dam break case, $\gamma-S P H-A L E$ provides solution in very good agreements with the experiment even with violent flow conditions (impact, free surface reconnection). The total energy is again preserved thanks to an efficient control on the artificial diffusion through the scheme parameters.

$\gamma-S P H-A L E$ is then able to provide accurate results in both compressible and weakly compressible frameworks, disregarding any low-Mach limitations. Thanks to a stable and consistent combination with regularizing techniques as PST and dissipative tools, $\gamma-S P H-A L E$ can endure violent free surface flows while preserving a correct energy balance. Contrary to RSALE, $\gamma-S P H-A L E$ provides both stable and accurate results without drastically increase the solving complexity, and then the computation times.

\section{References}

[1] J. P. Vila, On Particle Weighted Methods And Smooth Particle Hydrodynamics, Mathematical Models and Methods in Applied Sciences 09 (1999) 161-209.

[2] N. Grenier, J. P. Vila, P. Villedieu, An Accurate low-Mach Scheme for a Compressible Two-fluid Model Applied to Free-surface Flows, Journal Computational Physics 252 (2013) 1-19.

[3] G. Lavalle, J.-P. Vila, G. Blanchard, C. Laurent, F. Charru, A Numerical Reduced Model for Thin Liquid Films Sheared by a Gas Flow, Joachim Computational Physics 301 (2015) 119-140.

[4] L. B. Lucy, A numerical approach to the testing of the fission hypothesis 82 (1977) 1013-1024.

[5] J. J. Monaghan, R. A. Gingold, Shock Simulation by the Particle Method SPH, Journal of Computational Physics 52 (1983) 374-389.

[6] J. Monaghan, Simulating Free Surface Flows with SPH, Journal of Computational Physics 110 (1994) $399-406$.

[7] J. J. Monaghan, Smoothed Particle Hydrodynamics 30 (1992) 543-574.

[8] W. Benz, Smooth Particle Hydrodynamics: A Review, Springer Netherlands, Dordrecht, 1990, pp. 269-288.

[9] W. Benz, E. Asphaug, Impact Simulations with Fracture. I. Method and Tests, Icarus 107 (1994) 98-116.

[10] E. S. Lee, C. Moulinec, R. Xu, D. Violeau, D. Laurence, P. Stansby, Comparisons of Weakly Compressible and Truly Incompressible Algorithms for the SPH Mesh Free Particle Method, Journal Computational Physics 227 (2008) 8417-8436.

[11] R. Fatehi, M. T. Manzari, A Remedy for Numerical Oscillations in Weakly Compressible Smoothed Particle Hydrodynamics, International Journal for Numerical Methods in Fluids 67 (2011) 1100-1114. 
[12] A. Colagrossi, M. Landrini, Numerical Simulation of Interfacial Flows by Smoothed Particle Hydrodynamics, Journal of Computational Physics 191 (2003) 448-475.

[13] X. Y. Hu, N. A. Adams, A Multi-phase SPH Method for Macroscopic and Mesoscopic Flows, Journal of Computational Physics 213 (2) (2006) 844-861.

[14] J. J. Monaghan, A. Rafiee, A simple SPH algorithm for multi-fluid flow with high density ratios 71 (2013) $537-561$.

[15] G. R. Johnson, S. R. Beissel, Normalized Smoothing Functions For Sph Impact Computations, International Journal for Numerical Methods in Engineering 39 (1996) 2725-2741.

[16] Vila, Jean-Paul, Methodes particulaires regularisees. Developpements recents et nouvelles applications , ESAIM: Proceedings 3 (1998) 131-146.

[17] G. A. Dilts, Moving least squares particle hydrodynamics I. Consistency and stability, International Journal for Numerical Methods in Engineering 44 (8) (1999) 1115-1155.

[18] N. Frontiere, C. D. Raskin, J. M. Owen, CRKSPH : A Conservative Reproducing Kernel Smoothed Particle Hydrodynamics Scheme, Journal of Computational Physics 332 (2017) 160 - 209.

[19] N. Lanson, J.-P. Vila, Renormalized Meshfree Schemes I: Consistency, Stability, and Hybrid Methods for Conservation Laws, SIAM Journal on Numerical Analysis 46 (4) (2008) 1912-1934.

[20] S. Marrone, M. Antuono, A. Colagrossi, G. Colicchio, D. L. Touze, G. Graziani, $\delta$-SPH model for simulating violent impact flows, Computer Methods in Applied Mechanics and Engineering 200 (2011) 1526 - 1542.

[21] D. Molteni, A. Colagrossi, A simple procedure to improve the pressure evaluation in hydrodynamic context using the SPH, Computer Physics Communications 180 (2009) 861-872.

[22] M. Antuono, A. Colagrossi, S. Marrone, Numerical diffusive terms in weakly-compressible SPH schemes, Computer Physics Communications 183 (2012) 2570-2580.

[23] A. Ferrari, M. Dumbser, E. F. Toro, A. Armanini, A new 3D parallel SPH scheme for free surface flows, Computers and Fluids 38 (2009) 1203-1217.

[24] J. Monaghan, SPH Without a Tensile Instability, Journal of Computational Physics 159 (2000) 290-311.

[25] J. Swegle, D. Hicks, S. Attaway, Smoothed Particle Hydrodynamics Stability Analysis, Journal of Computational Physics 116 (1995) 123-134.

[26] J. Monaghan, On the problem of penetration in particle methods, Journal of Computational Physics 82 (1) (1989) $1-15$.

[27] R. Xu, P. Stansby, D. Laurence, Accuracy and stability in incompressible SPH (ISPH) based on the projection method and a new approach, Journal of Computational Physics 228 (2009) $6703-6725$.

[28] S. Lind, R. Xu, P. Stansby, B. Rogers, Incompressible Smoothed Particle Hydrodynamics for free-surface flows: A generalised diffusion-based algorithm for stability and validations for impulsive flows and propagating waves, Journal of Computational Physics 231 (2012) 1499-1523.

[29] S. Adami, X. Hu, N. Adams, A transport-velocity formulation for Smoothed Particle Hydrodynamics, Journal of Computational Physics 241 (2013) $292-307$.

[30] G. Oger, S. Marrone, D. L. Touze, M. de Leffe, SPH Accuracy Improvement through the Combination of a Quasi-Lagrangian Shifting Transport Velocity and Consistent ALE Formalisms , Journal of Computational Physics 313 (2016) 76-98.

[31] A. Khayyer, H. Gotoh, Y. Shimizu, Comparative study on accuracy and conservation properties of two particle regularization schemes and proposal of an optimized particle shifting scheme in ISPH context, Journal of Computational Physics 332 (2017) 236 - 256.

[32] P. Sun, A. Colagrossi, S. Marrone, A. Zhang, The $\delta$-plus SPH model: Simple procedures for a further improvement of the SPH scheme, Computer Methods in Applied Mechanics and Engineering 315 (2017) $25-49$. 
[33] C. Zhang, X. Y. Hu, N. A. Adams, A generalized transport-velocity formulation for Smoothed Particle Hydrodynamics, Journal of Computational Physics 337 (2017) $216-232$.

[34] D. L. Touze, A. Colagrossi, G. Colicchio, M. Greco, A critical investigation of Smoothed Particle Hydrodynamics applied to problems with free surfaces, International Journal for Numerical Methods in Fluids 73 (7) 660-691.

[35] C. Hirt, A. Amsden, J. Cook, An Arbitrary Lagrangian-Eulerian Computing Method for All Flow Speeds, Journal of Computational Physics 135 (1997) 203-216.

[36] C. W. Hirt, An arbitrary Lagrangian-Eulerian Computing Technique, in: M. Holt (Ed.), Numerical Methods in Fluid Dynamics, Vol. 8 of Lecture Notes in Physics, Berlin Springer Verlag, 1971, pp. 350-355.

[37] W. Noh, CEL: A Time-Dependent, Two-Space-Dimensional, Coupled Eulerian-Lagrange Code, 1963.

[38] S. Lind, P. Stansby, High order Eulerian Incompressible Smoothed Particle Hydrodynamics with Transition to Lagrangian Free-Surface Motion, Journal of Computational Physics 326 (2016) 290 - 311.

[39] G. Fourtakas, P. Stansby, B. Rogers, S. Lind, An Eulerian Lagrangian Incompressible SPH formulation (ELI-SPH) connected with a sharp interface, Computer Methods in Applied Mechanics and Engineering 329 (2018) $532-552$.

[40] E. F. Toro, Riemann solvers and numerical methods for fluid dynamics : a practical introduction, Springer, Berlin, New York, 1997.

[41] P. Degond, M. Tang, All Speed Scheme for the Low Mach Number Limit of the Isentropic Euler Equations, Communications in Computational Physics 10 (2011) 1-31.

[42] H. Guillard, C. Viozat, On the behaviour of upwind schemes in the low Mach number limit, Computers and Fluids 28 (1) (1999) $63-86$.

[43] F. Couderc, A. Duran, J.-P. Vila, An explicit asymptotic preserving low Froude scheme for the multilayer shallow water model with density stratification, Journal of Computational Physics 343 (Supplement C) (2017) $235-270$

[44] D. Violeau, A. Leroy, On the maximum time step in weakly compressible SPH, Journal of Computational Physics 256 (Supplement C) (2014) $388-415$

[45] M. Clayer, J. Lacome, J. Limido, J. Vila, Pressure Evaluation Improvement for Euler Isentropic SPH Scheme, in: Proceedings of the 2014 9th international SPHERIC workshop, Paris, France, 2014.

[46] C.-W. Shu, S. Osher, Efficient implementation of essentially non-oscillatory shock-capturing schemes, Journal of Computational Physics 77 (2) (1988) $439-471$.

[47] C. Zhang, X. Hu, N. Adams, A Weakly Compressible SPH Method Based on a Low dissipation Riemann Solver, Journal of Computational Physics 335 (C) (2017) 605-620.

[48] S. Marrone, A. Colagrossi, D. L. Touze, G. Graziani, Fast free-surface detection and level-set function definition in SPH solvers, Journal of Computational Physics 229 (10) (2010) 3652 - 3663.

[49] J.-C. Marongiu, Methode numerique lagrangienne pour la simulation d'ecoulements a surface libre : application aux turbines Pelton, Theses, Ecole Centrale de Lyon (2007).

[50] J. Leduc, Etude physique et numerique de l'ecoulement dans un dispositif d'injection de turbine Pelton, Theses, Ecole Centrale de Lyon (Dec 2010).

[51] A. Colagrossi, A meshless Lagrangian method for free-surface and interface flows with fragmentation, These, Universita di Roma.

[52] M. Antuono, S. Marrone, A. Colagrossi, B. Bouscasse, Energy balance in the $\delta$-SPH scheme, Computer Methods in Applied Mechanics and Engineering 289 (2015) 209 - 226. 


\section{Appendix A - Robustness}

\subsection{Statement}

We can exhibit a CFL condition defining density bounds. Indeed, enforcing the condition

$$
\Delta t \frac{\|\boldsymbol{w}\|_{\infty}}{h} \leq \min _{n, i}\left(N_{\rho}^{i, n}\right)
$$

where

$$
N_{\rho}^{i, n}=\left(C_{i}^{n}\left[\sup _{j}\left(\left|\frac{\rho_{i j}^{n}}{\rho_{i}^{n}}\right|\right)+\frac{\gamma^{n}}{2 c_{0}\|\boldsymbol{w}\|_{\infty}} \sup _{j}\left(\left|\frac{R_{i j}^{n}}{\rho_{i}^{n}}\right|\left|\delta P_{i j}^{n}\right|\right)\right]\right)^{-1}
$$

with the following definitions

$$
\left\{\begin{array}{l}
C_{i}^{n} \text { defined as }(16) \\
\rho_{i j}^{n}=\rho_{i}^{n}+\rho_{j}^{n} \\
R_{i j}^{n}=\frac{\rho_{i j}^{n}}{\tilde{\rho}_{i j}^{n}} \\
\overline{\boldsymbol{w}}_{i j}^{n}=\frac{1}{2}\left(\boldsymbol{w}_{i}^{n}+\boldsymbol{w}_{j}^{n}\right) \\
\delta P_{i j}^{n}=p_{j}^{n}-p_{i}^{n}
\end{array}\right.
$$

insures that

$$
\rho_{i, \min }(t) \leq \rho_{i}(t) \leq \rho_{i, \max }(t)
$$

with

$$
\left\{\begin{array} { c } 
{ \rho _ { i , \operatorname { m i n } } ( t ) = \rho _ { i } ^ { 0 } e ^ { - C _ { \operatorname { m i n } } t } } \\
{ \rho _ { i , \operatorname { m a x } } ( t ) = \rho _ { i } ^ { 0 } e ^ { C _ { \operatorname { m a x } } t } }
\end{array} \text { and } \left\{\begin{array}{c}
C_{\min }=\frac{1}{\Delta t} \ln \left(2 \frac{\omega_{\max }}{\omega_{\min }}\right) \\
C_{\max }=\frac{1}{\Delta t} \ln \left(\frac{3}{2} \frac{\omega_{\max }}{\omega_{\min }}\right)
\end{array}\right.\right.
$$

\subsection{Proof}

Volume inequality According to the volume equation in (25) which is

$$
\frac{d \omega_{i}}{d t}=\omega_{i} \operatorname{div}\left(\boldsymbol{v}_{0}\left(\boldsymbol{x}_{i}, t\right)\right)
$$

as $\boldsymbol{v}_{0}$ is an imposed field, we completely control the volume evolution. It means that we can wisely choose it in order to insure volume bounds as follows

$$
\exists\left(\omega_{\min }, \omega_{\max }\right) \in \mathbb{R}^{+*}, \forall t, \omega_{\min } \leq \omega_{i}(t) \leq \omega_{\max }
$$

Density inequality We now set $\boldsymbol{F}_{i j}^{n}=\left(\rho_{i}^{n}+\rho_{j}^{n}\right) \boldsymbol{w}_{i j}^{n}=\rho_{i j}^{n} \overline{\boldsymbol{w}}_{i j}^{n}-\rho_{i j}^{n} \gamma_{i j}^{n} \delta P_{i j}^{n} \boldsymbol{n}_{i j}$ with $\rho_{i j}^{n}=\rho_{i}^{n}+\rho_{j}^{n}, \overline{\boldsymbol{w}}_{i j}^{n}=$ $\frac{1}{2}\left(\boldsymbol{w}_{i}^{n}+\boldsymbol{w}_{j}^{n}\right)$.

Taking $\gamma_{i j}^{n}=\frac{\gamma^{n} \widetilde{\kappa}_{i j}^{\prime n}}{2 c_{0}^{3}}=\frac{\gamma^{n}}{2 c_{0} \tilde{\rho}_{i j}}$ we can write

$$
\boldsymbol{F}_{i j}^{n}=\rho_{i j}^{n} \frac{\overline{\boldsymbol{w}}_{i j}^{n}}{2}-\frac{\gamma^{n}}{2 c_{0}} R_{i j}^{n} \delta P_{i j}^{n} \boldsymbol{n}_{i j}^{n}
$$

with $R_{i j}^{n}=\frac{\rho_{i j}^{n}}{\tilde{\rho}_{i j}^{n}}$.

We can now rewrite the mass scheme as

$$
\rho_{i}^{n+1} w_{i}^{n+1}-\rho_{i}^{n} w_{i}^{n}=-\Delta t w_{i}^{n} \sum_{j} w_{j}^{n}\left\langle\boldsymbol{F}_{i j}^{n}, \boldsymbol{A}_{i j}^{n}\right\rangle
$$

The objective is to ensure thanks to a CFL condition that

$$
\rho_{i}^{n+1} w_{i}^{n+1} \geq \Delta t w_{i}^{n} \sum_{j}-w_{j}^{n}\left\langle\boldsymbol{F}_{i j}^{n}, \boldsymbol{A}_{i j}^{n}\right\rangle^{-} \geq 0
$$

We know that

$$
\begin{aligned}
\Delta t w_{i}^{n} \sum_{j}-w_{j}^{n}\left\langle\boldsymbol{F}_{i j}^{n}, \boldsymbol{A}_{i j}^{n}\right\rangle^{-} & \leq \Delta t w_{i}^{n} \sum_{j} w_{j}^{n}\left|\boldsymbol{F}_{i j}^{n} \cdot \boldsymbol{A}_{i j}^{n}\right| \\
\rho_{i}^{n+1} w_{i}^{n+1} & \geq \rho_{i}^{n} w_{i}^{n}-\Delta t w_{i}^{n} \sum_{j} w_{j}^{n}\left|\boldsymbol{F}_{i j}^{n} \cdot \boldsymbol{A}_{i j}^{n}\right|
\end{aligned}
$$

Then 


$$
\begin{aligned}
\rho_{i}^{n+1} w_{i}^{n+1}-\Delta t w_{i}^{n} \sum_{j}-w_{j}^{n}\left\langle\boldsymbol{F}_{i j}^{n}, \boldsymbol{A}_{i j}^{n}\right\rangle^{-} \geq & \rho_{i}^{n} w_{i}^{n}-2 \Delta t w_{i}^{n} \sum_{j} w_{j}^{n}\left|\boldsymbol{F}_{i j}^{n} \cdot \boldsymbol{A}_{i j}^{n}\right| \\
\geq & \rho_{i}^{n} w_{i}^{n} \\
& -\Delta t w_{i}^{n} \sum_{j} w_{j}^{n}\left|\rho_{i j}^{n} \overline{\boldsymbol{w}}_{i j}^{n} \cdot \boldsymbol{A}_{i j}^{n}\right| \\
& -\frac{\gamma}{2 c_{0}} \Delta t w_{i}^{n} \sum_{j} w_{j}^{n}\left|R_{i j}^{n}\right|\left|\delta P_{i j}^{n}\right|\left\|\boldsymbol{A}_{i j}^{n}\right\|
\end{aligned}
$$

A sufficient condition to get the positivity is to ensure

$$
\begin{gathered}
\Delta t \sum_{j} w_{j}^{n}\left|\rho_{i j}^{n} \overline{\boldsymbol{w}}_{i j}^{n} \cdot \boldsymbol{A}_{i j}^{n}\right|+\frac{\gamma}{2 c_{0}} \Delta t \sum_{j} w_{j}^{n}\left|R_{i j}^{n}\right|\left|\delta P_{i j}^{n}\right|\left\|\boldsymbol{A}_{i j}^{n}\right\| \leq \rho_{i}^{n} \\
\Delta t \sum_{j} w_{j}^{n}\left(\left|\frac{\rho_{i j}^{n}}{\rho_{i}^{n}} \overline{\boldsymbol{w}}_{i j}^{n} \cdot \boldsymbol{A}_{i j}^{n}\right|+\frac{\gamma^{n}}{2 c_{0}}\left|\frac{R_{i j}^{n}}{\rho_{i}^{n}}\right|\left|\delta P_{i j}^{n}\right|\left\|\boldsymbol{A}_{i j}^{n}\right\|\right) \leq 1 \\
\Delta t \sum_{j} w_{j}^{n}\left(\left|\frac{\rho_{i j}^{n}}{\rho_{i}^{n}}\right|\left\|\overline{\boldsymbol{w}}_{i j}^{n}\right\|+\frac{\gamma^{n}}{2 c_{0}}\left|\frac{R_{i j}^{n}}{\rho_{i}^{n}}\right|\left|\delta P_{i j}^{n}\right|\right)\left\|\boldsymbol{A}_{i j}^{n}\right\| \leq 1
\end{gathered}
$$

Thus, using the definition of $C_{i}^{n}$, ensuring

$$
\Delta t \leq\left(\frac{C_{i}^{n}}{h}\left[\sup _{j}\left(\left|\frac{\rho_{i j}^{n}}{\rho_{i}^{n}}\right|\left\|\overline{\boldsymbol{w}}_{i j}^{n}\right\|\right)+\frac{\gamma^{n}}{2 c_{0}} \sup _{j}\left(\left|\frac{R_{i j}^{n}}{\rho_{i}^{n}}\right|\left|\delta P_{i j}^{n}\right|\right)\right]\right)^{-1}
$$

gives the expected density positivity

$$
\rho_{i}^{n+1} w_{i}^{n+1} \geq 0 \text { and then } \rho_{i}^{n+1} \geq 0
$$

Note that we have

$$
\rho_{i}^{n} w_{i}^{n}-\Delta t w_{i}^{n} \sum_{j} w_{j}^{n}\left|\boldsymbol{F}_{i j}^{n} \cdot \boldsymbol{A}_{i j}^{n}\right| \leq \rho_{i}^{n+1} w_{i}^{n+1} \leq \rho_{i}^{n} w_{i}^{n}+\Delta t w_{i}^{n} \sum_{j} w_{j}^{n}\left|\boldsymbol{F}_{i j}^{n} \cdot \boldsymbol{A}_{i j}^{n}\right|
$$

Also under the CFL condition (51) we have

$$
\rho_{i}^{n} w_{i}^{n} \geq 2 \Delta t w_{i}^{n} \sum_{j} w_{j}^{n}\left|\boldsymbol{F}_{i j}^{n} \cdot \boldsymbol{A}_{i j}^{n}\right|
$$

meaning that

$$
\left\{\begin{array}{l}
\Delta t w_{i}^{n} \sum_{j} w_{j}^{n}\left|\boldsymbol{F}_{i j}^{n} \cdot \boldsymbol{A}_{i j}^{n}\right| \leq \frac{1}{2} \rho_{i}^{n} w_{i}^{n} \\
-\frac{1}{2} \rho_{i}^{n} w_{i}^{n} \leq-\Delta t w_{i}^{n} \sum_{j} w_{j}^{n}\left|\boldsymbol{F}_{i j}^{n} \cdot \boldsymbol{A}_{i j}^{n}\right|
\end{array}\right.
$$

Hence

$$
\frac{1}{2} \rho_{i}^{n} w_{i}^{n} \leq \rho_{i}^{n+1} w_{i}^{n+1} \leq \frac{3}{2} \rho_{i}^{n} w_{i}^{n}
$$

Giving, according to the volume bounds,

$$
\left(\frac{1}{2} \frac{\omega_{\min }}{\omega_{\max }}\right)^{n+1} \rho_{i}^{0} \leq \rho_{i}^{n+1} \leq\left(\frac{3}{2} \frac{\omega_{\max }}{\omega_{\min }}\right)^{n+1} \rho_{i}^{0}
$$

And finally with $\rho_{i}^{0}>0$, in a continuous framework,

$$
\forall t, 0<\rho_{i, \min }(t) \leq \rho_{i}(t) \leq \rho_{i, \max }(t)
$$

where

$$
\left\{\begin{array} { c } 
{ \rho _ { i , \operatorname { m i n } } ( t ) = \rho _ { i } ^ { 0 } e ^ { - C _ { \operatorname { m i n } } t } } \\
{ \rho _ { i , \operatorname { m i n } } ( t ) = \rho _ { i } ^ { 0 } e ^ { C _ { \operatorname { m a x } } t } }
\end{array} \text { and } \left\{\begin{array}{l}
C_{\min }=\frac{1}{\Delta t} \ln \left(2 \frac{\omega_{\max }}{\omega_{\min }}\right) \\
C_{\text {max }}=\frac{1}{\Delta t} \ln \left(\frac{3}{2} \frac{\omega_{\max }}{\omega_{\min }}\right)
\end{array}\right.\right.
$$




\section{Appendix B - Energy Balance}

\subsection{Statement}

Under the following stability conditions

$$
\text { for } \Delta t \frac{c_{0}}{h} \leq \frac{1}{C} \min _{n}\left(N_{\alpha}^{n}, N_{\gamma}^{n}\right), \forall(\alpha, \gamma) \in\left[\alpha_{\min }, \alpha_{\max }\right] \mathrm{x}\left[\gamma_{\min }, \gamma_{\max }\right]
$$

Such that we can show

$$
\forall 0 \leq t \leq T, \exists \mathcal{E}_{T} \geq 0, \mathcal{E}(t) \leq \mathcal{E}_{T}
$$

which ensures the bounded behavior of the scheme total energy and gives the expected stability property.

Note that we have

$$
\begin{gathered}
\left\{\begin{array}{l}
N_{\gamma}^{n}=\frac{\sqrt{2 \eta\left[\left(M_{w}^{n}\right)^{2}(2 \eta+1)+1\right]}-2 \eta M_{w}^{n}}{8 \eta^{2}\left(\left(M_{w}^{n}\right)^{2}+1\right)} \\
N_{\alpha}^{n}=\frac{\sqrt{2\left[\left(M_{w}^{n}\right)^{2}+\eta\right]}-M_{w}^{n}}{4 \eta\left(\left(M_{w}^{n}\right)^{2}+2 \eta\right)}
\end{array}\right. \\
\left\{\begin{array} { l } 
{ \gamma _ { \text { min } } = \operatorname { m a x } _ { n } \gamma _ { \text { min } } ^ { n } } \\
{ \gamma _ { \text { max } } = \operatorname { m i n } _ { n } \gamma _ { \text { max } } ^ { n } }
\end{array} \text { and } \left\{\begin{array}{l}
\alpha_{\text {min }}=\max _{n} \alpha_{\text {min }}^{n} \\
\alpha_{\text {max }}=\min _{n} \alpha_{\text {max }}^{n}
\end{array}\right.\right. \\
\left\{\begin{array} { l } 
{ \gamma _ { \text { min } } ^ { n } = \frac { 1 - \sqrt { 1 - 4 a b } } { 2 a } } \\
{ \gamma _ { \text { max } } ^ { n } = \frac { 1 + \sqrt { 1 - 4 a b } } { 2 a } }
\end{array} \text { with } \left\{\begin{array}{l}
b=M_{w}^{n}\left(2 N^{n} M_{w}^{n} \eta+1\right)+2 N^{n} \eta \\
a=4 N^{n} \eta^{2}
\end{array}\right.\right. \\
\left\{\begin{array} { l } 
{ \alpha _ { \text { min } } ^ { n } = \frac { 1 - \sqrt { 1 - a ^ { \prime } b ^ { \prime } } } { a ^ { \prime } } } \\
{ \alpha _ { \text { max } } ^ { n } = \frac { 1 + \sqrt { 1 - a ^ { \prime } b ^ { \prime } } } { a ^ { \prime } } }
\end{array} \quad \text { with } \left\{\begin{array}{l}
b^{\prime}=M_{w}^{n}\left(2 N^{n} M_{w}^{n} \eta+1\right)+4 N^{n} \eta^{2} \\
a_{T}^{\prime}=8 N^{n} \eta
\end{array}\right.\right. \\
\mathcal{E}_{0}+C_{1} T+o(1) \text { when } \Delta t \rightarrow 0
\end{gathered}
$$

where $N^{n}=\Delta t \frac{c_{0}}{h} C^{n}, M_{w}^{n}=\frac{\|\boldsymbol{w}\|_{\infty}^{n}}{c_{0}}$ and $C$ as $(16), \mathcal{E}_{0}$ the initial total energy, $C_{1}$ a constant (depending on the geometry, the sound speed and the arbitrary velocity field) and $T$ the endtime.

1. An energy balance is performed on the scheme to write the discrete energy equation, and the production terms coming from the kinetic and potential energy are exhibited

2. These terms are estimated and gathered to provide a global estimation of the energy production

3. A negativity condition on one part of the production estimate provides stability conditions on $\alpha$, $\gamma$ and $\Delta t$

4. The remaining production terms are evaluated under such stability conditions and provide a finite energy bound noted $\mathcal{E}_{T}$

\subsection{Proof}

\subsubsection{Definitions}

We firstly define the total energy $E$ as $E=\frac{1}{2} \rho\|v\|^{2}+\varphi$ where $\varphi=\rho \int_{\rho_{0}}^{\rho} \frac{p(r)}{r^{2}} d r$ is the total free energy and corresponds to the barotropic pressure. We set $\kappa=\varphi^{\prime}=\frac{d \varphi}{d \rho}$. Secondly, being in a barotropic context we work with a linear equation of state written as $p=c_{0}^{2}\left(\rho-\rho_{0}\right)$ where $\rho_{0}, c_{0}$ are the characteristic material density and sound speed.

\subsubsection{Discrete energy equation}

Let consider the following conservation equations discretized with a first order time integration scheme

$$
\begin{gathered}
\boldsymbol{x}_{i}^{n+1}-\boldsymbol{x}_{i}^{n}=\mathbf{v}_{\mathbf{0 i}}^{\mathbf{n}} \Delta t \\
w_{i}^{n+1}-w_{i}^{n}=\Delta t w_{i}^{n} \sum_{j}\left\langle\left(\boldsymbol{v}_{0 j}^{n}-\boldsymbol{v}_{0 i}^{n}\right), \boldsymbol{A}_{i j}^{n}\right\rangle w_{j}^{n} \\
\rho_{i}^{n+1} w_{i}^{n+1}-\rho_{i}^{n} w_{i}^{n}=-\Delta t w_{i}^{n} \sum_{j} w_{j}^{n}\left(\rho_{j}^{n}+\rho_{i}^{n}\right)\left\langle\mathbf{w}_{\boldsymbol{i j}}^{\boldsymbol{n}}, \boldsymbol{A}_{\boldsymbol{i j}}^{\boldsymbol{n}}\right\rangle
\end{gathered}
$$




$$
\begin{aligned}
\rho_{i}^{n+1} w_{i}^{n+1} \boldsymbol{v}_{i}^{n+1}-\rho_{i}^{n} w_{i}^{n} \boldsymbol{v}_{i}^{n}= & -\Delta t w_{i}^{n} \sum_{j} w_{j}^{n}\left(\rho_{j}^{n} \boldsymbol{v}_{j}^{n}+\rho_{i}^{n} \boldsymbol{v}_{i}^{n}\right)\left\langle\boldsymbol{w}_{i j}^{n}, \boldsymbol{A}_{\boldsymbol{i j}}^{n}\right\rangle \\
& -2 \Delta t w_{i}^{n} \sum_{j} w_{j}^{n} p_{i j}^{n} \boldsymbol{A}_{i j}^{n} \\
& +2 \Delta t w_{i}^{n} \boldsymbol{\Pi}_{i}
\end{aligned}
$$

where $\boldsymbol{\Pi}_{i}=\sum_{j} w_{j}^{n} \boldsymbol{\pi}_{i j}$.

Scheme on $v$ Using the mass (62) and momentum (63) equations we get on $v$

$$
\begin{aligned}
\boldsymbol{v}_{i}^{n+1}= & \boldsymbol{v}_{i}^{n}+\Delta t \frac{w_{i}^{n}}{w_{i}^{n+1}} \sum_{j} w_{j}^{n} \frac{\rho_{j}^{n}}{\rho_{i}^{n+1}}\left(\boldsymbol{v}_{i}^{n}-\boldsymbol{v}_{j}^{n}\right)\left\langle\boldsymbol{w}_{i j}^{n}, \boldsymbol{A}_{\boldsymbol{i j}}^{n}\right\rangle \\
& -2 \Delta t \frac{w_{i}^{n}}{w_{i}^{n+1} \rho_{i}^{n+1}} \sum_{j} w_{j}^{n} p_{i j}^{n} \boldsymbol{A}_{\boldsymbol{i j}}^{n}+2 \Delta t \frac{w_{i}^{n}}{w_{i}^{n+1} \rho_{i}^{n+1}} \boldsymbol{\Pi}_{i}
\end{aligned}
$$

Scheme on $\rho\|v\|^{2}$ Using the equality: $\boldsymbol{v}_{i}^{n}\left(\boldsymbol{v}_{i}^{n+1}-\boldsymbol{v}_{i}^{n}\right)=\frac{1}{2}\left\|\boldsymbol{v}_{i}^{n+1}\right\|^{2}-\frac{1}{2}\left\|\boldsymbol{v}_{i}^{n}\right\|^{2} \frac{1}{2}\left\|\boldsymbol{v}_{i}^{n+1}-\boldsymbol{v}_{i}^{n}\right\|^{2}$, the mass scheme (62) and the velocity scheme (64) we get

$$
\begin{aligned}
\frac{1}{2} w_{i}^{n+1} \rho_{i}^{n+1}\left\|\boldsymbol{v}_{i}^{n+1}\right\|^{2} & -\frac{1}{2} \rho_{i}^{n} w_{i}^{n}\left\|\boldsymbol{v}_{i}^{n}\right\|^{2} \\
= & \frac{1}{2} w_{i}^{n+1} \rho_{i}^{n+1}\left\|\boldsymbol{v}_{i}^{n+1}-\boldsymbol{v}_{i}^{n}\right\|^{2} \\
& +\Delta t w_{i}^{n} \sum_{j} w_{j}^{n} \rho_{j}^{n} \frac{1}{2}\left\|\boldsymbol{v}_{j}^{n}-\boldsymbol{v}_{i}^{n}\right\|^{2}\left\langle\boldsymbol{w}_{i j}^{n}, \boldsymbol{A}_{i j}^{n}\right\rangle \\
& -\Delta t w_{i}^{n} \sum_{j} w_{j}^{n}\left(\frac{1}{2}\left\|\boldsymbol{v}_{i}^{n}\right\|^{2} \rho_{i}^{n}+\frac{1}{2}\left\|\boldsymbol{v}_{j}^{n}\right\|^{2} \rho_{j}^{n}\right)\left\langle\boldsymbol{w}_{i j}^{n}, \boldsymbol{A}_{i j}^{n}\right\rangle \\
& -2 \Delta t w_{i}^{n} \boldsymbol{v}_{i}^{n} \sum_{j} w_{j}^{n} p_{i j}^{n} \boldsymbol{A}_{i j}^{n}+2 \Delta t w_{i}^{n}\left\langle\boldsymbol{v}_{i}^{n}, \boldsymbol{\Pi}_{i}\right\rangle
\end{aligned}
$$

Pressure part of the energy Regarding the pressure part we note that $\rho \kappa=\varphi(\rho)+p$. Besides, considering that Taylor's expansion of $\varphi$ gives certain $\theta_{i j}$ and $\theta_{i}$ in $[0,1]$ such that $\tilde{\kappa}_{i j}^{n \prime}=\kappa^{\prime}\left(\left(1-\theta_{i j}\right) \rho_{i}^{n}+\theta_{i j} \rho_{j}^{n}\right)$ and $\tilde{\kappa}_{i}^{n \prime}=\kappa^{\prime}\left(\left(1-\theta_{i}\right) \rho_{i}^{n}+\theta_{i} \rho_{i}^{n+1}\right)$ we have:

$$
\left\{\begin{array}{l}
\varphi\left(\rho_{j}^{n}\right)-\varphi\left(\rho_{i}^{n}\right)=\kappa_{i}^{n}\left(\rho_{j}^{n}-\rho_{i}^{n}\right)+\frac{1}{2} \tilde{\kappa}_{i j}^{n \prime}\left(\rho_{j}^{n}-\rho_{i}^{n}\right)^{2} \\
\varphi\left(\rho_{i}^{n+1}\right)-\varphi\left(\rho_{i}^{n}\right)=\kappa_{i}^{n}\left(\rho_{i}^{n+1}-\rho_{i}^{n}\right)+\frac{1}{2} \tilde{\kappa}_{i}^{n \prime}\left(\rho_{i}^{n+1}-\rho_{i}^{n}\right)^{2}
\end{array}\right.
$$

Multiplying the mass conservation (62) equation by $\kappa_{i}^{n}$ we can write

$$
\begin{aligned}
w_{i}^{n+1}\left(\varphi\left(\rho_{i}^{n+1}\right)+p_{i}^{n+1}\right) & -w_{i}^{n}\left(\varphi\left(\rho_{i}^{n}\right)+p_{i}^{n}\right) \\
= & w_{i}^{n+1} \rho_{i}^{n+1}\left(\kappa_{i}^{n+1}-\kappa_{i}^{n}\right) \\
& -\Delta t w_{i}^{n} \sum_{j} w_{j}^{n}\left(\rho_{j}^{n}+\rho_{i}^{n}\right) \kappa_{i}^{n}\left\langle\mathbf{w}_{i j}^{n}, \boldsymbol{A}_{i j}^{n}\right\rangle \\
= & w_{i}^{n+1} \rho_{i}^{n+1}\left(\kappa_{i}^{n+1}-\kappa_{i}^{n}\right) \\
& -\Delta t w_{i}^{n} \sum_{j} w_{j}^{n}\left(\rho_{j}^{n}-\rho_{i}^{n}\right) \kappa_{i}^{n}\left\langle\mathbf{w}_{i j}^{n}, \boldsymbol{A}_{i j}^{n}\right\rangle \\
& -2 \Delta t w_{i}^{n} \sum_{j} w_{j}^{n} \rho_{i}^{n} \kappa_{i}^{n}\left\langle\mathbf{w}_{i j}^{n}, \boldsymbol{A}_{i j}^{n}\right\rangle
\end{aligned}
$$

Replacing $\rho_{i}^{n} \kappa_{i}^{n}$ and $\left(\rho_{j}^{n}-\rho_{i}^{n}\right) \kappa_{i}^{n}$ thanks to (66) we get with $\mathbf{w}_{i j}^{n}=\mathbf{v}_{i j}^{n}-\boldsymbol{v}_{0 i j}^{n}$, where $\boldsymbol{v}_{0 i j}^{n}=\frac{\left(\boldsymbol{v}_{9 i}^{n}+\boldsymbol{v}_{n_{0 j}}\right)}{2}$ and $\mathbf{v}_{i j}^{n}=\frac{\left(\boldsymbol{v}_{i}^{n}+\boldsymbol{v}_{j}^{n}\right)}{2}-\gamma_{i j}^{n}\left(p_{j}^{n}-p_{i}^{n}\right) \boldsymbol{n}_{i j}$

$$
\begin{aligned}
w_{i}^{n+1} \varphi\left(\rho_{i}^{n+1}\right)-w_{i}^{n} \varphi\left(\rho_{i}^{n}\right) & +\Delta t w_{i}^{n} \sum_{j}\left(\varphi\left(\rho_{j}^{n}\right)+\varphi\left(\rho_{i}^{n}\right)\right) w_{j}^{n}\left\langle\mathbf{w}_{i j}^{n}, \boldsymbol{A}_{i j}^{n}\right\rangle \\
& +2 \Delta t w_{i}^{n} p_{i}^{n} \sum_{j}\left\langle\mathbf{v}_{i j}^{n}, \boldsymbol{A}_{i j}^{n}\right\rangle w_{j}^{n} \\
= & -p_{i}^{n}\left(w_{i}^{n+1}-w_{i}^{n}-\Delta t w_{i}^{n} \sum_{j} 2\left\langle\boldsymbol{v}_{0 i j}^{n}, \boldsymbol{A}_{i j}^{n}\right\rangle w_{j}^{n}\right) \\
& +w_{i}^{n+1}\left(p_{i}^{n}-p_{i}^{n+1}+\rho_{i}^{n+1}\left(\kappa_{i}^{n+1}-\kappa_{i}^{n}\right)\right) \\
& +\Delta t w_{i}^{n} \sum_{j}\left\langle\mathbf{w}_{i j}^{n}, \boldsymbol{A}_{i j}^{n}\right\rangle \frac{1}{2} \widetilde{\kappa}_{i j}^{\prime n}\left(\rho_{i}^{n}-\rho_{j}^{n}\right)^{2} w_{j}^{n}
\end{aligned}
$$

And having $p_{i}^{n}-p_{i}^{n+1}+\rho_{i}^{n+1}\left(\kappa_{i}^{n+1}-\kappa_{i}^{n}\right)=\frac{1}{2} \widetilde{\kappa}_{i}^{\prime n}\left(\rho_{i}^{n+1}-\rho_{i}^{n}\right)^{2}$ leads to

$$
\begin{aligned}
w_{i}^{n+1} \varphi\left(\rho_{i}^{n+1}\right)-w_{i}^{n} \varphi\left(\rho_{i}^{n}\right) & +\Delta t w_{i}^{n} \sum_{j}\left(\varphi\left(\rho_{j}^{n}\right)+\varphi\left(\rho_{i}^{n}\right)\right) w_{j}^{n}\left\langle\mathbf{w}_{i j}^{n}, \boldsymbol{A}_{i j}^{n}\right\rangle \\
& +2 \Delta t w_{i}^{n} p_{i}^{n} \sum_{j}\left\langle\mathbf{v}_{i j}^{n}, \boldsymbol{A}_{\boldsymbol{i j}}^{n}\right\rangle w_{j}^{n} \\
= & -p_{i}^{n}\left(\left(w_{i}^{n+1}-w_{i}^{n}\right)-2 \Delta t w_{i}^{n} \sum_{j}\left\langle\boldsymbol{v}_{\mathbf{0 i j}}^{n}, \boldsymbol{A}_{\boldsymbol{i j}}^{n}\right\rangle w_{j}^{n}\right) \\
& +w_{i}^{n+1} \frac{1}{2} \widetilde{\kappa}_{i}^{n}\left(\rho_{i}^{n+1}-\rho_{i}^{n}\right)^{2} \\
& +\Delta t w_{i}^{n} \sum_{j}\left\langle\mathbf{w}_{i j}^{n}, \boldsymbol{A}_{i j}^{n}\right\rangle \frac{1}{2} \widetilde{\kappa}_{i j}^{\prime n}\left(\rho_{i}^{n}-\rho_{j}^{n}\right)^{2} w_{j}^{n}
\end{aligned}
$$


Total Energy Gathering (65) and (67) gives

$$
\begin{aligned}
& w_{i}^{n+1}\left(\frac{1}{2} \rho_{i}^{n+1}\left\|\boldsymbol{v}_{\boldsymbol{i}}^{\boldsymbol{n}+\mathbf{1}}\right\|^{2}+\varphi\left(\rho_{i}^{n+1}\right)\right)-w_{i}^{n}\left(\frac{1}{2} \rho_{i}^{n}\left\|\boldsymbol{v}_{\boldsymbol{i}}^{\boldsymbol{n}}\right\|^{2}+\varphi\left(\rho_{i}^{n}\right)\right) \\
& +w_{i}^{n} \Delta t \sum_{j}\left(\frac{1}{2} \rho_{i}^{n}\left\|\boldsymbol{v}_{\boldsymbol{i}}^{\boldsymbol{n}}\right\|^{2}+\varphi\left(\rho_{i}^{n}\right)\right) w_{j}^{n}\left\langle\mathbf{w}_{i j}^{n}, \boldsymbol{A}_{i j}^{n}\right\rangle+w_{i}^{n} 2 \Delta t v_{i}^{n} \sum_{j} p_{i j}^{n} \boldsymbol{A}_{i j}^{n} w_{j}^{n} \\
& +w_{i}^{n} \Delta t \sum_{j}\left(\frac{1}{2} \rho_{j}^{n}\left\|\boldsymbol{v}_{j}^{n}\right\|^{2}+\varphi\left(\rho_{j}^{n}\right)\right) w_{j}^{n}\left\langle\mathbf{w}_{i j}^{n}, \boldsymbol{A}_{i j}^{n}\right\rangle+w_{i}^{n} 2 \Delta t p_{i}^{n} \sum_{j}\left\langle\boldsymbol{v}_{\boldsymbol{i j}}^{\boldsymbol{n}}, \boldsymbol{A}_{\boldsymbol{i} \boldsymbol{j}}^{\boldsymbol{n}}\right\rangle w_{j}^{n} \\
& -w_{i}^{n} 2 \Delta t\left\langle\boldsymbol{v}_{i}^{n}, \boldsymbol{\Pi}_{i}\right\rangle \\
= & -p_{i}^{n+1}\left(\left(w_{i}^{n+1}-w_{i}^{n}\right)-2 \Delta t w_{i}^{n} \sum_{j}\left\langle\boldsymbol{v}_{\mathbf{0 i j}}^{\boldsymbol{n}}, \boldsymbol{A}_{\boldsymbol{i j}}^{\boldsymbol{n}}\right\rangle w_{j}^{n}\right) \\
& +w_{i}^{n} \Delta t \sum_{j}\left\langle\mathbf{w}_{i j}^{n}, \boldsymbol{A}_{i j}^{n}\right\rangle \frac{1}{2} \widetilde{\kappa}_{i j}^{\prime n}\left(\rho_{i}^{n}-\rho_{j}^{n}\right)^{2} w_{j}^{n}+w_{i}^{n+1} \frac{1}{2} \widetilde{\kappa}_{i}^{\prime n}\left(\rho_{i}^{n+1}-\rho_{i}^{n}\right)^{2} \\
& +w_{i}^{n} \Delta t \sum_{j}\left\langle\mathbf{w}_{i j}^{n}, \boldsymbol{A}_{i j}^{n}\right\rangle \rho_{j}^{n} \frac{1}{2}\left\|\boldsymbol{v}_{\boldsymbol{j}}^{\boldsymbol{n}}-\boldsymbol{v}_{\boldsymbol{i}}^{n}\right\|^{2} w_{j}^{n}+w_{i}^{n+1} \frac{1}{2} \rho_{i}^{n+1}\left\|\boldsymbol{v}_{\boldsymbol{i}}^{\boldsymbol{n}+\mathbf{1}}-\boldsymbol{v}_{\boldsymbol{i}}^{\boldsymbol{n}}\right\|^{2}
\end{aligned}
$$

Considering that

$$
\begin{aligned}
v_{i}^{n} \sum_{j} p_{i j}^{n} \boldsymbol{A}_{i j}^{n} w_{j}^{n} & -\left\langle\boldsymbol{v}_{i}^{n}, \boldsymbol{\Pi}_{i}\right\rangle+p_{i}^{n} \sum_{j}\left\langle\boldsymbol{v}_{i j}^{n}, \boldsymbol{A}_{i j}^{n}\right\rangle w_{j}^{n} \\
= & \sum_{j} \frac{1}{2}\left(\boldsymbol{v}_{i}^{n} p_{j}^{n}+\boldsymbol{v}_{j}^{n} p_{i}^{n}\right) \boldsymbol{A}_{i j}^{n} w_{j}^{n}+\boldsymbol{v}_{i}^{n} p_{i}^{n} \sum_{j} \boldsymbol{A}_{i j}^{n} w_{j}^{n} \\
& -\frac{1}{2} \sum_{j} \gamma_{i j}^{n}\left(p_{j}^{n}+p_{i}^{n}\right)\left(p_{j}^{n}-p_{i}^{n}\right)\left\|\boldsymbol{A}_{i j}^{n}\right\| w_{j}^{n} \\
& +\frac{1}{2} \sum_{j} \gamma_{i j}^{n}\left(p_{j}^{n}-p_{i}^{n}\right)^{2}\left\|\boldsymbol{A}_{i j}^{n}\right\| w_{j}^{n} \\
& -\frac{1}{2} \sum_{j} \alpha_{i j}^{n}\left(\boldsymbol{v}_{j}^{n}+\boldsymbol{v}_{i}^{n}\right)\left(\boldsymbol{v}_{j}^{n}-\boldsymbol{v}_{i}^{n}\right) w_{j}^{n} \\
& +\frac{1}{2} \sum_{j} \alpha_{i j}^{n}\left\|\boldsymbol{v}_{j}^{n}-\boldsymbol{v}_{i}^{n}\right\|^{2} w_{j}^{n}
\end{aligned}
$$

We can finally write

$$
\begin{aligned}
& w_{i}^{n+1} E_{i}^{n+1}-w_{i}^{n} E_{i}^{n}+w_{i}^{n} \Delta t\left(\sum_{j}\left(E_{i}^{n}+E_{j}^{n}\right)\left\langle\mathbf{w}_{i j}^{n}, \boldsymbol{A}_{i j}^{n}\right\rangle w_{j}^{n}\right) \\
& +\frac{1}{2} w_{i}^{n} 2 \Delta t \sum_{j}\left(v_{i}^{n} p_{j}^{n}+p_{i}^{n} v_{j}^{n}\right) \boldsymbol{A}_{i j}^{n} w_{j}^{n} \\
& -2 \Delta t w_{i}^{n} \frac{1}{2} \sum_{j} \gamma_{i j}^{n}\left(p_{j}^{n}+p_{i}^{n}\right)\left(p_{j}^{n}-p_{i}^{n}\right)\left\|\boldsymbol{A}_{i j}^{n}\right\| w_{j}^{n} \\
& -2 \Delta t w_{i}^{n} \frac{1}{2} \sum_{j} \alpha_{i j}^{n}\left\langle\left(\boldsymbol{v}_{\boldsymbol{j}}^{n}+\boldsymbol{v}_{\boldsymbol{i}}^{n}\right),\left(\boldsymbol{v}_{\boldsymbol{j}}^{n}-\boldsymbol{v}_{\boldsymbol{i}}^{n}\right)\right\rangle w_{j}^{n} \\
= & \Delta t S_{i}^{n}+w_{i}^{n+1}\left(Q_{i}^{n}+R_{i}^{n}\right)
\end{aligned}
$$

where

$$
\begin{aligned}
& Q_{i}^{n}=\frac{1}{2} \rho_{i}^{n+1}\left\|\boldsymbol{v}_{\boldsymbol{i}}^{\boldsymbol{n}+\mathbf{1}}-\boldsymbol{v}_{\boldsymbol{i}}^{\boldsymbol{n}}\right\|^{2} \\
& +\frac{\Delta t . w_{i}^{n}}{w_{i}^{n+1}} \sum_{j}\left\langle\mathbf{w}_{i j}^{n}, \boldsymbol{A}_{i j}^{n}\right\rangle \rho_{j}^{n} \frac{1}{2}\left\|\boldsymbol{v}_{\boldsymbol{j}}^{\boldsymbol{n}}-\boldsymbol{v}_{\boldsymbol{i}}^{\boldsymbol{n}}\right\|^{2} w_{j}^{n} \\
& -\frac{\Delta t w_{i}^{n}}{w_{i}^{n+1}} \sum_{j} \gamma_{i j}^{n}\left(p_{j}^{n}-p_{i}^{n}\right)^{2}\left\|\boldsymbol{A}_{i j}^{n}\right\| w_{j}^{n} \\
& R_{i}^{n}=\frac{1}{2} \widetilde{\kappa}_{i}^{\prime n}\left(\rho_{i}^{n+1}-\rho_{i}^{n}\right)^{2} \\
& +\frac{1}{2} \frac{\Delta t \cdot w_{i}^{n}}{w_{i}^{n+1}} \sum_{j}\left\langle\mathbf{w}_{i j}^{n}, \boldsymbol{A}_{i j}^{n}\right\rangle \widetilde{\kappa}_{i j}^{\prime n}\left(\rho_{i}^{n}-\rho_{j}^{n}\right)^{2} w_{j}^{n} \\
& -\frac{\Delta t w_{i}^{n}}{w_{i}^{n+1}} \sum_{j} \alpha_{i j}^{n}\left\|\boldsymbol{v}_{j}^{n}-\boldsymbol{v}_{\boldsymbol{i}}^{n}\right\|^{2} w_{j}^{n} \\
& \Delta t S_{i}^{n}=-p_{i}^{n}\left(\left(w_{i}^{n+1}-w_{i}^{n}\right)-2 \Delta t w_{i}^{n} \sum_{j}\left\langle\boldsymbol{v}_{\mathbf{0 i j}}^{\boldsymbol{n}}, \boldsymbol{A}_{\boldsymbol{i j}}^{\boldsymbol{n}}\right\rangle w_{j}^{n}\right)
\end{aligned}
$$

\subsubsection{Estimation of the production terms}

Estimation of $\Delta t S_{i}^{n} \quad$ Considering the volume equation (61) we have 


$$
\begin{aligned}
\Delta t S_{i}^{n} & =2 \Delta t w_{i}^{n} p_{i}^{n} \sum_{j}\left\langle\boldsymbol{v}_{\mathbf{0} \boldsymbol{i}}^{n}, \boldsymbol{A}_{\boldsymbol{i j}}^{n}\right\rangle w_{j}^{n} \\
& =2 \Delta t w_{i}^{n} p_{i}^{n}\left\langle\boldsymbol{v}_{0 i}^{n}, \sum_{j} \boldsymbol{A}_{\boldsymbol{i} \boldsymbol{j}}^{n} w_{j}^{n}\right\rangle
\end{aligned}
$$

Thus, $K_{1}$ definition (16) gives

$$
\Delta t S_{i}^{n} \leq K_{1} \Delta t w_{i}^{n} p_{i}^{n}\left\|v_{0 i}^{n}\right\|
$$

Estimation of $Q_{i}^{n} \quad$ Using the scheme on $\boldsymbol{v}(64)$ and Cauchy-Schwarz' inequality we can write:

$$
\begin{aligned}
& \frac{1}{2} \rho_{i}^{n+1}\left\|\boldsymbol{v}_{i}^{n+1}-\boldsymbol{v}_{i}^{n}\right\|^{2} \\
\leq & \rho_{i}^{n+1}\left(\frac{\Delta t w_{i}^{n}}{w_{i}^{n+1} \rho_{i}^{n+1}}\right)^{2}\left(\sum_{j}\left|\left\langle\mathbf{w}_{i j}^{n}, \boldsymbol{A}_{i j}^{n}\right\rangle\right| \rho_{j}^{n}\left\|\boldsymbol{v}_{j}^{n}-\boldsymbol{v}_{i}^{n}\right\|^{2} w_{j}^{n}\right)\left(\sum_{j}\left|\left\langle\mathbf{w}_{i j}^{n}, \boldsymbol{A}_{i j}^{n}\right\rangle\right| \rho_{j}^{n} w_{j}^{n}\right) \\
& +\rho_{i}^{n+1}\left(\frac{\Delta t w_{i}^{n}}{w_{i}^{n+1} \rho_{i}^{n+1}}\right)^{2}\left(\sum_{j}\left(p_{j}^{n}-p_{i}^{n}\right)^{2}\left\|\boldsymbol{A}_{i j}^{n}\right\| w_{j}^{n}\right)\left(\sum_{j}\left\|\boldsymbol{A}_{i j}^{n}\right\| w_{j}^{n}\right) \\
& +\rho_{i}^{n+1}\left(\frac{2 \Delta t w_{i}^{n}}{w_{i}^{n+1} \rho_{i}^{n+1}}\right)^{2}\left(p_{i}^{n}\right)^{2}\left(\sum_{j} \boldsymbol{A}_{i j}^{n} w_{j}^{n}\right)^{2} \\
& +\rho_{i}^{n+1}\left(\frac{2 \Delta t w_{i}^{n}}{w_{i}^{n+1} \rho_{i}^{n+1}}\right)^{2}\left(\sum_{j} \frac{\alpha_{i j}^{2}}{\left\|\boldsymbol{A}_{i j}^{n}\right\|^{2}}\left\|\boldsymbol{v}_{j}^{n}-\boldsymbol{v}_{i}^{n}\right\|^{2}\left\|\boldsymbol{A}_{i j}^{n}\right\| w_{j}^{n}\right)\left(\sum_{j}\left\|\boldsymbol{A}_{i j}^{n}\right\| w_{j}^{n}\right)
\end{aligned}
$$

Leading to

$$
\begin{aligned}
Q_{i}^{n} & \\
\leq & \frac{w_{i}^{n}}{w_{i}^{n+1}} \Delta t\left(\sum_{j} \frac{1}{2}\left|\left\langle\mathbf{w}_{i j}^{n}, \boldsymbol{A}_{i j}^{n}\right\rangle\right| \rho_{j}^{n}\left\|\boldsymbol{v}_{j}^{n}-\boldsymbol{v}_{\boldsymbol{i}}^{n}\right\|^{2} w_{j}^{n}(1\right. \\
& \left.\left.\quad+2\left(\sum_{j}\left|\left\langle\mathbf{w}_{i j}^{n}, \boldsymbol{A}_{i j}^{n}\right\rangle\right| \rho_{j}^{n} w_{j}^{n}\right) \frac{1}{\rho_{i}^{n+1}}\left(\Delta t \frac{w_{i}^{n}}{w_{i}^{n+1}}\right)\right)\right) \\
& -\frac{w_{i}^{n}}{w_{i}^{n+1}} \Delta t\left(\sum_{j} \gamma_{i j}\left(p_{j}^{n}-p_{i}^{n}\right)^{2}\left\|\boldsymbol{A}_{i j}^{n}\right\| w_{j}^{n}(1\right. \\
& \left.\left.\quad-\left(\sum_{j}\left\|\boldsymbol{A}_{i j}^{n}\right\| w_{j}^{n}\right) \frac{1}{\gamma_{i j} \rho_{i}^{n+1}}\left(\Delta t \frac{w_{i}^{n}}{w_{i}^{n+1}}\right)\right)\right) \\
& +\frac{1}{\rho_{i}^{n+1}}\left(2 \Delta t \frac{w_{i}^{n}}{w_{i}^{n+1}}\right)^{2}\left(\sum_{j} \frac{\alpha_{i j}^{2}}{\left\|\boldsymbol{A}_{i j}^{n}\right\|}\left\|\boldsymbol{v}_{j}^{n}-\boldsymbol{v}_{i}^{n}\right\|^{2} w_{j}^{n}\right)\left(\sum_{j}\left\|\boldsymbol{A}_{i j}^{n}\right\| w_{j}^{n}\right) \\
& +\frac{1}{\rho_{i}^{n+1}}\left(2 \Delta t \frac{w_{i}^{n}}{w_{i}^{n+1}}\right)^{2}\left(p_{i}^{n}\right)^{2}\left(\sum_{j} \boldsymbol{A}_{i j}^{n} w_{j}^{n}\right)^{2}
\end{aligned}
$$

Estimation of $R_{i}^{n} \quad$ According to the mass scheme (62) we have

$$
\begin{aligned}
\rho_{i}^{n+1}-\rho_{i}^{n}= & -\frac{w_{i}^{n+1}-w_{i}^{n}}{w^{n+1}} \rho_{i}^{n} \\
& -\frac{w_{n}^{n}}{w_{i}^{n+1}} \Delta t \sum_{j}\left\langle\mathbf{w}_{i j}^{n}, \boldsymbol{A}_{i j}^{n}\right\rangle\left(\rho_{j}^{n}-\rho_{i}^{n}\right) w_{j}^{n} \\
& -2 \Delta t \frac{w_{i}^{n}}{w_{i}^{n+1}} \rho_{i}^{n} \sum_{j}\left\langle\mathbf{w}_{i j}^{n}, \boldsymbol{A}_{i j}^{n}\right\rangle w_{j}^{n}
\end{aligned}
$$

Using again Cauchy-Schwarz' inequality we can write

$$
\begin{aligned}
R_{i}^{n} & \Delta t \frac{w_{i}^{n}}{w_{i}^{n+1}}\left(\sum_{j} \frac{1}{2} \widetilde{\kappa}_{i j}^{\prime n}\left|\left\langle\mathbf{w}_{i j}^{n}, \boldsymbol{A}_{i j}^{n}\right\rangle\right|\left(\rho_{j}^{n}-\rho_{i}^{n}\right)^{2} w_{j}^{n}(1\right. \\
& \left.\left.\quad+2 \frac{\widetilde{\kappa}_{i}^{\prime n}}{\widetilde{\kappa}_{i j}^{\prime n}} \Delta t \frac{w_{i}^{n}}{w_{i}^{n+1}}\left(\sum_{j}\left|\left\langle\mathbf{w}_{i j}^{n}, \boldsymbol{A}_{i j}^{n}\right\rangle\right| w_{j}^{n}\right)\right)\right)^{2} \\
& +\widetilde{\kappa}_{i}^{\prime n}\left(\rho_{i}^{n}\right)^{2}\left(2 \Delta t \frac{w_{i}^{n}}{w_{i}^{n+1}}\right)^{2}\left(\sum_{j}\left\langle\mathbf{w}_{i j}^{n}, \boldsymbol{A}_{i j}^{n}\right\rangle w_{j}^{n}\right)^{2} \\
& +\widetilde{\kappa}_{i}^{\prime n}\left(\rho_{i}^{n}\right)^{2} \frac{\left(w_{i}^{n+1}-w_{i}^{n+1}\right)^{2}}{\left(w_{i}^{n+1}\right)^{2}} \\
& -\frac{w_{i}^{n}}{w_{i}^{n+1}} \Delta t \sum_{j} \alpha_{i j}\left\|\boldsymbol{v}_{j}^{n}-\boldsymbol{v}_{\boldsymbol{i}}^{n}\right\|^{2} w_{j}^{n}
\end{aligned}
$$

Considering that 


$$
\begin{aligned}
\left(\sum_{j}\left\langle\mathbf{w}_{i j}^{n}, \boldsymbol{A}_{i j}^{n}\right\rangle w_{j}^{n}\right)^{2}= & \left(\sum_{j}\left\langle\left(\mathbf{v}_{i j}^{n}-\boldsymbol{v}_{0 i j}^{n}\right), \boldsymbol{A}_{i j}^{n}\right\rangle w_{j}^{n}\right)^{2} \\
\leq & \frac{1}{2}\left(\sum_{j}\left\|\boldsymbol{v}_{j}^{n}-\boldsymbol{v}_{\boldsymbol{i}}^{n}\right\|^{2} w_{j}^{n}\left\|\boldsymbol{A}_{i j}^{n}\right\|\right)\left(\sum_{j} w_{j}^{n}\left\|\boldsymbol{A}_{i j}^{n}\right\|\right) \\
& +2\left(\sum_{j} \gamma_{i j}^{2}\left(p_{j}^{n}-p_{i}^{n}\right)^{2} w_{j}^{n}\left\|\boldsymbol{A}_{i j}^{n}\right\|\right)\left(\sum_{j} w_{j}^{n}\left\|\boldsymbol{A}_{i j}^{n}\right\|\right) \\
& +2\left\|\boldsymbol{v}_{i}^{n}\right\|^{2}\left(\sum_{j} w_{j}^{n} \boldsymbol{A}_{i j}^{n}\right)^{2}+2\left(\sum_{j} w_{j}^{n}\left\langle\boldsymbol{v}_{\mathbf{0} i j}^{n}, \boldsymbol{A}_{\boldsymbol{i j}}^{n}\right\rangle\right)^{2} \\
\leq & \frac{1}{2}\left(\sum_{j}\left\|\boldsymbol{v}_{j}^{n}-\boldsymbol{v}_{i}^{n}\right\|^{2} w_{j}^{n}\left\|\boldsymbol{A}_{i j}^{n}\right\|\right)\left(\sum_{j} w_{j}^{n}\left\|\boldsymbol{A}_{i j}^{n}\right\|\right) \\
& +2\left(\sum_{j} \gamma_{i j}^{2}\left(p_{j}^{n}-p_{i}^{n}\right)^{2} w_{j}^{n}\left\|\boldsymbol{A}_{i j}^{n}\right\|\right)^{2}\left(\sum_{j} w_{j}^{n}\left\|\boldsymbol{A}_{i j}^{n}\right\|\right) \\
& +4\left(\frac{\left(w_{i}^{n+1}-w_{i}^{n}\right)}{2 \Delta t w_{i}^{n}}\right)^{2} \\
& +\left(\sum_{j} w_{j}^{n} \boldsymbol{A}_{i j}^{n}\right)^{2} 2\left(\left\|\boldsymbol{v}_{i}^{n}\right\|^{2}+2\left\|v_{0 i}^{n}\right\|^{2}\right)
\end{aligned}
$$

We get

$$
\begin{aligned}
& R_{i}^{n} \\
\leq & \Delta t \frac{w_{i}^{n}}{w_{i}^{n+1}}\left(\sum_{j} \frac{1}{2} \widetilde{\kappa}_{i j}^{\prime n}\left|\left\langle\mathbf{w}_{i j}^{n}, \boldsymbol{A}_{i j}^{n}\right\rangle\right|\left(\rho_{j}^{n}-\rho_{i}^{n}\right)^{2} w_{j}^{n}(1\right. \\
& \left.\left.\quad+2 \frac{\widetilde{\kappa}_{i}^{n}}{\widetilde{\kappa}_{i j}^{n}} \Delta t \frac{w_{i}^{n}}{w_{i}^{n+1}}\left(\sum_{j}\left|\left\langle\mathbf{w}_{i j}^{n}, \boldsymbol{A}_{i j}^{n}\right\rangle\right| w_{j}^{n}\right)\right)\right) \\
& -\frac{w_{i}^{n}}{w_{i}^{n+1}} \Delta t \sum_{j} \alpha_{i j}\left\|\boldsymbol{v}_{j}^{n}-\boldsymbol{v}_{i}^{n}\right\|^{2} w_{j}^{n} \\
& +\widetilde{\kappa}_{i}^{\prime n}\left(\rho_{i}^{n}\right)^{2}\left(2 \Delta t \frac{w_{n}^{n}}{w_{i}^{n}+1}\right)^{2} \frac{1}{2}\left(\sum_{j}\left\|\boldsymbol{v}_{j}^{n}-\boldsymbol{v}_{i}^{n}\right\|^{2} w_{j}^{n}\left\|\boldsymbol{A}_{i j}^{n}\right\|\right)\left(\sum_{j} w_{j}^{n}\left\|\boldsymbol{A}_{i j}^{n}\right\|\right) \\
& +\widetilde{\kappa}_{i}^{\prime n}\left(\rho_{i}^{n}\right)^{2}\left(2 \Delta t \frac{w_{i}^{n}}{w_{i}^{n+1}}\right)^{2} 2\left(\sum_{j} \gamma_{i j}^{2}\left(p_{j}^{n}-p_{i}^{n}\right)^{2} w_{j}^{n}\left\|\boldsymbol{A}_{i j}^{n}\right\|\right)\left(\sum_{j} w_{j}^{n}\left\|\boldsymbol{A}_{i j}^{n}\right\|\right) \\
& +5 \widetilde{\kappa}_{i}^{\prime n}\left(\rho_{i}^{n}\right)^{2}\left(\frac{w_{i}^{n+1}-w_{i}^{n}}{w_{i}^{n+1}}\right)^{2} \\
& +2\left(\left\|\boldsymbol{v}_{i}^{n}\right\|^{2}+2\left\|v_{0 i}^{n}\right\|^{2}\right) \widetilde{\kappa}_{i}^{\prime n}\left(\rho_{i}^{n}\right)^{2}\left(2 \Delta t \frac{w_{i}^{n}}{w_{i}^{n+1}}\right)^{2}\left(\sum_{j} w_{j}^{n} \boldsymbol{A}_{i j}^{n}\right)^{2}
\end{aligned}
$$

Estimation of $Q_{i}^{n}+R_{i}^{n} \quad$ Gathering now equations (73) and (74) gives

$$
\begin{aligned}
& Q_{i}^{n}+R_{i}^{n} \\
& \leq \Delta t \frac{w_{i}^{n}}{w_{i}^{n+1}}\left(\sum_{j} \widetilde{\kappa}_{i j}^{\prime n} \frac{1}{2}\left|\left\langle\mathbf{w}_{i j}^{n}, \boldsymbol{A}_{i j}^{n}\right\rangle\right|\left(\rho_{j}^{n}-\rho_{i}^{n}\right)^{2} w_{j}^{n}(1\right. \\
& \left.\left.+2 \frac{\widetilde{\kappa}_{i}^{\prime n}}{\widetilde{\kappa}_{i j}^{\prime n}} \Delta t \frac{w_{i}^{n}}{w_{i}^{n+1}}\left(\sum_{j}\left|\left\langle\mathbf{w}_{i j}^{n}, \boldsymbol{A}_{i j}^{n}\right\rangle\right| w_{j}^{n}\right)\right)\right) \\
& -\frac{w_{i}^{n}}{w_{i}^{n+1}} \Delta t\left(\sum_{j} \gamma_{i j}\left(p_{j}^{n}-p_{i}^{n}\right)^{2}\left\|\boldsymbol{A}_{i j}^{n}\right\| w_{j}^{n}(1\right. \\
& \left.\left.-\left(\sum_{j}\left\|\boldsymbol{A}_{i j}^{n}\right\| w_{j}^{n}\right)\left(\frac{1}{\gamma_{i j} \rho_{i}^{n+1}}+8 . \widetilde{\kappa}_{i}^{\prime n}\left(\rho_{i}^{n}\right)^{2} \gamma_{i j}\right)\left(\Delta t \frac{w_{i}^{n}}{w_{i}^{n+1}}\right)\right)\right) \\
& +\frac{w_{i}^{n}}{w_{i}^{n+1}} \Delta t\left(\sum_{j} \frac{1}{2}\left|\left\langle\mathbf{w}_{i j}^{n}, \boldsymbol{A}_{i j}^{n}\right\rangle\right| \rho_{j}^{n}\left\|\boldsymbol{v}_{\boldsymbol{j}}^{n}-\boldsymbol{v}_{\boldsymbol{i}}^{n}\right\|^{2} w_{j}^{n}(1\right. \\
& \left.\left.+2 .\left(\sum_{j}\left|\left\langle\mathbf{w}_{i j}^{n}, \boldsymbol{A}_{i j}^{n}\right\rangle\right| \frac{\rho_{j}^{n}}{\rho_{i}^{n+1}} w_{j}^{n}\right)\left(\Delta t \frac{w_{i}^{n}}{w_{i}^{n+1}}\right)\right)\right) \\
& -\Delta t \frac{w_{i}^{n}}{w_{i}^{n+1}}\left(\sum_{j} \alpha_{i j}\left\|\boldsymbol{v}_{j}^{n}-\boldsymbol{v}_{\boldsymbol{i}}^{n}\right\|^{2} w_{j}^{n}(1\right. \\
& \left.\left.-\left(4 \frac{\alpha_{i j}}{\rho_{i}^{n+1} \cdot\left\|\boldsymbol{A}_{i j}^{n}\right\|}+2 \cdot \frac{\widetilde{\kappa}_{i}^{\prime n}\left(\rho_{i j}^{n}\right)^{2}}{\alpha_{i j}} \cdot\left\|\boldsymbol{A}_{i j}^{n}\right\|\right)\left(\sum_{j}\left\|\boldsymbol{A}_{i j}^{n}\right\| w_{j}^{n}\right) \Delta t \frac{w_{i}^{n}}{w_{i}^{n+1}}\right)\right) \\
& +\left(2 \Delta t \frac{w_{i}^{n}}{w_{i}^{n+1}}\right)^{2}\left(\frac{\left(p_{i}^{n}\right)^{2}}{\rho_{i}^{n+1}}+2\left(\left\|\boldsymbol{v}_{i}^{n}\right\|^{2}+2\left\|v_{0 i}^{n}\right\|^{2}\right) \cdot \widetilde{\kappa}_{i}^{\prime n}\left(\rho_{i}^{n}\right)^{2}\right)\left(\sum_{j} w_{j}^{n} \boldsymbol{A}_{i j}^{n}\right)^{2} \\
& +5 . \widetilde{\kappa}_{i}^{\prime n}\left(\rho_{i}^{n}\right)^{2} \frac{\left(w_{i}^{n+1}-w_{i}^{n+1}\right)^{2}}{\left(w_{i}^{n+1}\right)^{2}}
\end{aligned}
$$

We can actually divide this inequality (75) in three parts: the first part contains the first and second rhs terms, the second parts contains the third and fourth rhs terms and the third part contains the last two terms. At this stage we want that the first and second parts be negative to ensure a dissipative behavior. 
- The first part

$$
\begin{aligned}
& \left(\sum_{j} \widetilde{\kappa}_{i j}^{\prime n} \frac{1}{2}\left|\left\langle\mathbf{w}_{i j}^{n}, \boldsymbol{A}_{i j}^{n}\right\rangle\right|\left(\rho_{j}^{n}-\rho_{i}^{n}\right)^{2} w_{j}^{n}\left(1+2 \frac{\widetilde{\kappa}_{i}^{\prime n}}{\widetilde{\kappa}_{i j}^{\prime n}} \Delta t \frac{w_{i}^{n}}{w_{i}^{n+1}}\left(\sum_{j}\left|\left\langle\mathbf{w}_{i j}^{n}, \boldsymbol{A}_{i j}^{n}\right\rangle\right| w_{j}^{n}\right)\right)\right) \\
& \leq\left(\sum_{j} \gamma_{i j}\left(p_{j}^{n}-p_{i}^{n}\right)^{2}\left\|\boldsymbol{A}_{i j}^{n}\right\| w_{j}^{n}\left(1-\left(\sum_{j}\left\|\boldsymbol{A}_{i j}^{n}\right\| w_{j}^{n}\right)\left(\frac{1}{\gamma_{i j} \rho_{i}^{n+1}}+8 \widetilde{\kappa}_{i}^{\prime n}\left(\rho_{i}^{n}\right)^{2} \gamma_{i j}\right)\left(\Delta t \frac{w_{i}^{n}}{w_{i}^{n+1}}\right)\right)\right)
\end{aligned}
$$

is reduced to

$$
\Delta t \frac{w_{i}^{n}}{w_{i}^{n+1}} q^{n}\left(\gamma^{n}\right) \sum_{j}\left[\frac{\widetilde{\kappa}_{i j}^{\prime n}}{2} c_{0}\left(\rho_{j}^{n}-\rho_{i}^{n}\right)^{2}\left\|\boldsymbol{A}_{i j}^{n}\right\| w_{j}^{n}\right] \leq 0
$$

by taking

$$
q^{n}\left(\gamma^{n}\right)=4 N^{n} \eta^{2}\left(\gamma^{n}\right)^{2}-\gamma^{n}+M_{w}^{n}\left(2 N^{n} M_{w}^{n} \eta+1\right)+2 N^{n} \eta \leq 0
$$

where $N^{n}=\frac{\Delta t c_{0}}{h} C^{n}, C$ as $(16), \gamma_{i j}^{n}=\gamma^{n} \frac{\widetilde{\kappa}_{i j}^{\prime n}}{2 \tilde{c}_{i j}^{n^{3}}}=\frac{\gamma^{n}}{2 c_{0} \tilde{\rho}_{i j}^{n}}, \eta=\frac{\rho_{\max }}{\rho_{\min }}$ and $M_{w}^{n}=\frac{\|w\|_{\infty}^{n}}{c_{0}}$.

- The second part

$$
\begin{aligned}
& \left(\sum_{j} \frac{1}{2}\left|\left\langle\mathbf{w}_{i j}^{n}, \boldsymbol{A}_{i j}^{n}\right\rangle\right| \rho_{j}^{n}\left\|\boldsymbol{v}_{j}^{n}-\boldsymbol{v}_{\boldsymbol{i}}^{n}\right\|^{2} w_{j}^{n}\left(1+2 \cdot\left(\sum_{j}\left|\left\langle\mathbf{w}_{i j}^{n}, \boldsymbol{A}_{i j}^{n}\right\rangle\right| \frac{\rho_{j}^{n}}{\rho_{i}^{n+1}} w_{j}^{n}\right)\left(\Delta t \frac{w_{i}^{n}}{w_{i}^{n+1}}\right)\right)\right) \\
& \leq\left(\sum_{j} \alpha_{i j}\left\|\boldsymbol{v}_{\boldsymbol{j}}^{n}-\boldsymbol{v}_{\boldsymbol{i}}^{n}\right\|^{2} w_{j}^{n}\left(1-\left(4 \frac{\alpha_{i j}}{\rho_{i}^{n+1} \cdot\left\|\boldsymbol{A}_{i j}^{n}\right\|}+2 \cdot \frac{\widetilde{\boldsymbol{\kappa}}_{i}^{\prime n}\left(\rho_{i j}^{n}\right)^{2}}{\alpha_{i j}} \cdot\left\|\boldsymbol{A}_{i j}^{n}\right\|\right)\left(\sum_{j}\left\|\boldsymbol{A}_{i j}^{n}\right\| w_{j}^{n}\right) \Delta t \frac{w_{i}^{n}}{w_{i}^{n+1}}\right)\right)
\end{aligned}
$$

is reduced to

$$
\Delta t \frac{w_{i}^{n}}{w_{i}^{n+1}} r^{n}\left(\alpha^{n}\right) \sum_{j}\left[\frac{\rho_{j}^{n}}{2} c_{0}\left\|\boldsymbol{v}_{j}^{n}-\boldsymbol{v}_{i}^{n}\right\|^{2}\left\|\boldsymbol{A}_{i j}^{n}\right\| w_{j}^{n}\right] \leq 0
$$

by taking

$$
r^{n}\left(\alpha^{n}\right)=8 N^{n} \eta\left(\alpha^{n}\right)^{2}-2 \alpha^{n}+M_{w}^{n}\left(2 N^{n} M_{w}^{n} \eta+1\right)+4 N^{n} \eta^{2} \leq 0
$$

with $\alpha_{i j}^{n}=\alpha^{n} \bar{c}_{i j}^{n} \bar{\rho}_{i j}^{n}\left\|\boldsymbol{A}_{i j}^{n}\right\|, \bar{c}_{i j}^{n}=c_{0}$ and $\bar{\rho}_{i j}^{n}=\frac{\rho_{i}^{n}+\rho_{j}^{n}}{2}$.

- Regarding the third part, we have the following terms

$$
\begin{array}{ll}
\text { (i) } & \left(2 \Delta t \frac{w_{i}^{n}}{w_{i}^{n+1}}\right)^{2}\left(\frac{\left(p_{i}^{n}\right)^{2}}{\rho_{i}^{n+1}}+4\left\|v_{0 i}^{n}\right\|^{2} . \widetilde{\kappa}_{i}^{\prime n}\left(\rho_{i}^{n}\right)^{2}\right)\left(\sum_{j} w_{j}^{n} \boldsymbol{A}_{i j}^{n}\right)^{2} \\
\text { (ii) } & 5 . \widetilde{\kappa}_{i}^{\prime n}\left(\rho_{i}^{n}\right)^{2} \frac{\left(w_{i}^{n+1}-w_{i}^{n+1}\right)^{2}}{\left(w_{i}^{n+1}\right)^{2}} \\
\text { (iii) } & 2\left\|\boldsymbol{v}_{i}^{n}\right\|^{2} \widetilde{\kappa}_{i}^{\prime n}\left(\rho_{i}^{n}\right)^{2}\left(2 \Delta t \frac{w_{i}^{n}}{w_{i}^{n+1}}\right)^{2}\left(\sum_{j} w_{j}^{n} \boldsymbol{A}_{i j}^{n}\right)^{2}
\end{array}
$$

Considering that $p_{i}^{n}=c_{0}^{2} \rho_{i}^{n}$ and $K_{1}$ definition (16)

$$
\begin{aligned}
(i) & =4 \Delta t^{2} c_{0}^{2}\left(c_{0}^{2} \frac{\rho_{i}^{n}}{\rho_{i}^{n+1}}+4\left\|v_{0 i}^{n}\right\|^{2} \frac{\rho_{i}^{n}}{\tilde{\rho}_{i}^{n}}\right) \rho_{i}^{n}\left(\sum_{j} w_{j}^{n} \boldsymbol{A}_{i j}^{n}\right)^{2} \\
& \leq 4 \Delta t^{2} c_{0}^{2} \eta\left(c_{0}^{2}+4\left\|v_{0}^{n}\right\|_{\infty}^{2}\right) K_{1}^{2} \rho_{i}^{n} \\
& \leq \Delta t^{2} K_{3} \rho_{i}^{n}
\end{aligned}
$$

where $K_{3 \eta}=4 c_{0}^{2} \eta\left(c_{0}^{2}+4\left\|v_{0}^{n}\right\|_{\infty}^{2}\right) K_{1}^{2}$

Also

$$
\begin{aligned}
(i i) & =5 \Delta t^{2} c_{0}^{2} \rho_{i}^{n}\left(d i v_{i} \boldsymbol{v}_{0}^{n}\right)^{2} \\
& \leq \Delta t^{2} K_{4} \rho_{i}^{n}
\end{aligned}
$$

where $K_{4}=5 c_{0}^{2}\left(\max _{i, n} d i v_{i} \boldsymbol{v}_{0}^{n}\right)^{2}$

Besides

$$
\begin{aligned}
(i i i) & \leq 8 K_{1}^{2} \Delta t^{2} c_{0}^{2} \rho_{i}^{n}\left\|\boldsymbol{v}_{i}^{n}\right\|^{2} \\
& \leq \Delta t^{2} K_{5}\left\|\boldsymbol{v}_{i}^{n}\right\|^{2} \rho_{i}^{n}
\end{aligned}
$$

where $K_{5}=8 K_{1}^{2} c_{0}^{2}$

Thus 


$$
\begin{aligned}
& (i)+(i i)+(i i i) \\
\leq & 4 \Delta t^{2} c_{0}^{2}\left(c_{0}^{2}+4\left\|v_{0}^{n}\right\|_{\infty}^{2}\right) K_{1}^{2} \rho_{i}^{n}+20 .\left(\frac{K_{3}}{h}\right)^{2} \Delta t^{2} c_{0}^{2}\left\|v_{0}^{n}\right\|_{\infty} \rho_{i}^{n}+8 K_{1}^{2} \Delta t^{2} c_{0}^{2} \rho_{i}^{n}\left\|\boldsymbol{v}_{i}^{n}\right\|^{2} \\
\leq & \Delta t^{2}\left(K_{3 \eta}+K_{4}+K_{5}\left\|\boldsymbol{v}_{i}^{n}\right\|^{2}\right) \rho_{i}^{n}
\end{aligned}
$$

Finally we get

$$
\begin{aligned}
Q_{i}^{n}+R_{i}^{n} \leq & \Delta t \frac{w_{i}^{n}}{w_{i}^{n+1}} q^{n}\left(\gamma^{n}\right) \sum_{j}\left[\frac{\widetilde{\kappa}_{i j}^{\prime n}}{2} c_{0}\left(\rho_{j}^{n}-\rho_{i}^{n}\right)^{2}\left\|\boldsymbol{A}_{i j}^{n}\right\| w_{j}^{n}\right] \\
& +\Delta t \frac{w_{i}^{n}}{w_{i}^{n+1}} r^{n}\left(\alpha^{n}\right) \sum_{j}\left[\frac{\rho_{j}^{n}}{2} c_{0}\left\|\boldsymbol{v}_{j}^{n}-\boldsymbol{v}_{i}^{n}\right\|^{2}\left\|\boldsymbol{A}_{i j}^{n}\right\| w_{j}^{n}\right] \\
& +\Delta t^{2}\left(K_{3}+K_{4}+K_{5}\left\|\boldsymbol{v}_{i}^{n}\right\|^{2}\right) \rho_{i}^{n}
\end{aligned}
$$

\subsubsection{Stability conditions}

The objective here, thanks to (76) and (77), is to explicit CFL-like conditions insuring the stability of the scheme with respect to $\alpha$ and $\gamma$.

$\gamma$ condition Regarding the expressions of $q^{n}\left(\gamma^{n}\right)(76)$, we can write the condition $q^{n}\left(\gamma^{n}\right) \leq 0$ as:

$$
a\left(\gamma^{n}\right)^{2}-\gamma^{n}+b \leq 0
$$

with $a=4 N^{n} \eta^{2}$ and $b=M_{w}^{n}\left(2 N^{n} M_{w}^{n} \eta+1\right)+2 N^{n} \eta$. We recall that $N^{n}=c_{0} \frac{\Delta t}{h} C^{n}$ where $C^{n}$ is defined as (16).

Thus, by setting $P=4 a b$, the condition $\Delta_{q}=1-P \geq 0$ read as $P \leq 1$ gives

$$
N^{n} \leq N_{\gamma}^{n}=\frac{\sqrt{2 \eta\left[\left(M_{w}^{n}\right)^{2}(2 \eta+1)+1\right]}-2 \eta M_{w}^{n}}{8 \eta^{2}\left(\left(M_{w}^{n}\right)^{2}+1\right)}
$$

we get the following admissible interval for $\gamma$

$$
\frac{1-\sqrt{1-P}}{2 a}=\gamma_{\min }^{n} \leq \gamma^{n} \leq \gamma_{\max }^{n}=\frac{1+\sqrt{1-P}}{2 a}
$$

- Stability Limit: We can define $N_{c r i t}^{n}=N_{\gamma}^{n}$ as the maximum admissible CFL to achieve the negativity of $q^{n}$. Thus taking $N^{n}=N_{c r i t}^{n}$ (corresponding to $\Delta_{q}=0$ ) gives as well a critical value $\gamma_{c r i t}^{n}=\frac{1}{2 a}=\frac{1}{8 \eta^{2} N_{c r i t}^{n}}$ insuring the negativity of $q^{n}$. Now in the low-Mach limit $\left(M \rightarrow 0\right.$ ie $M_{w} \rightarrow 0$ and $\left.\eta=1\right)$ we get $N_{\text {crit }}=\frac{\sqrt{2}}{8}$ and $\gamma_{c r i t}=\frac{1}{\sqrt{2}}$. However this value corresponds to a stability limit and does not minimize the amount of dissipation generated by the Low-Mach scheme (which is the expected behavior).

- Stability Field: Taking now $N^{n}<N_{c r i t}^{n}$, we can exhibit a minimum value for $\gamma^{n}$ in order to minimize the related dissipation. Knowing that $\forall x \in[0 ; 1], 1-\sqrt{1-x} \leq x$ and $1+\sqrt{1-x} \geq 1$, we have

$$
\frac{1-\sqrt{1-P}}{2 a} \leq \frac{P}{2 a} \text { and } \frac{1+\sqrt{1-P}}{2 a} \geq \frac{1}{2 a}
$$

Then, we choose to define in practice $\gamma_{\min }^{n}=2 b=2\left[M_{w}^{n}\left(2 N^{n} M_{w}^{n} \eta+1\right)+2 N^{n} \eta\right]$ and $\gamma_{\max }^{n}=\frac{1}{2 a}=\frac{1}{8 N^{n} \eta^{2}}$. Thus, working with $\gamma^{n}=\gamma_{\min }^{n}$ minimizes the related dissipation. In particular, in the Low-Mach limit we have $\gamma_{\min }^{n}=4 N^{n}=4 C^{n} \frac{\Delta t c_{0}}{h}$. Note that in [2], they use $\gamma_{e}$ (dimension of $\frac{1}{\rho c_{0}}$ ) linked to the present $\gamma$ (dimensionless) through $\gamma_{e}=\frac{\gamma}{\rho c_{0}}$. Thus we have $\frac{\gamma_{\min }^{n}}{\rho c_{0}}=4 C^{n} \frac{\Delta t}{\rho h}$ which is a similar stability condition as the one achieved in $[2,3,45]$.

$\alpha$ condition Regarding the expressions of $r^{n}\left(\alpha^{n}\right)(77)$, we can write the condition $r^{n}\left(\alpha^{n}\right) \leq 0$ as:

$$
a^{\prime}\left(\alpha^{n}\right)^{2}-2 \alpha^{n}+b^{\prime} \leq 0
$$

with $a^{\prime}=8 N^{n} \eta$ and $b^{\prime}=M_{w}^{n}\left(2 N^{n} M_{w}^{n} \eta+1\right)+4 N^{n} \eta^{2}$. 
Thus, by setting $P^{\prime}=a^{\prime} b^{\prime}$, the condition $\Delta_{r}=1-P^{\prime} \geq 0$ read as $P^{\prime} \leq 1$ gives

$$
N^{n} \leq N_{\alpha}^{n}=\frac{\sqrt{2\left[\left(M_{w}^{n}\right)^{2}+\eta\right]}-M_{w}^{n}}{4 \eta\left(\left(M_{w}^{n}\right)^{2}+2 \eta\right)}
$$

we get the following admissible interval for $\alpha$

$$
\frac{1-\sqrt{1-P^{\prime}}}{a^{\prime}}=\alpha_{\min }^{n} \leq \alpha^{n} \leq \alpha_{\max }^{n}=\frac{1+\sqrt{1-P^{\prime}}}{a^{\prime}}
$$

- Stability Limit: We can defin $\mathrm{e} N_{c r i t}^{n}=N_{\alpha}^{n}$ as the maximum admissible CFL to achieve the negativity of $r^{n}$. Thus taking $N^{n}=N_{c r i t}^{n}$ (corresponding to $\Delta_{r}=0$ ) gives as well a critical value $\alpha_{c r i t}^{n}=\frac{1}{a}=\frac{1}{8 \eta N_{c r i t}^{n}}$ insuring the negativity of $r^{n}$. Now in the low-Mach limit $\left(M \rightarrow 0\right.$ ie $M_{w} \rightarrow 0$ and $\left.\eta=1\right)$ we get $N_{c r i t}=\frac{\sqrt{2}}{8}$ and $\alpha_{c r i t}=\frac{1}{\sqrt{2}}$. However, similarly to $\gamma_{\text {crit }}$, this value corresponds to a stability limit and does not minimize the amount of dissipation generated by the artificial viscosity (which is the expected behavior).

- Stability Field: Taking now $N^{n}<N_{c r i t}^{n}$, we can exhibit a minimum value for $\alpha^{n}$ in order to minimize the related dissipation. Then, similarly to $\gamma$, we choose to define in practice $\alpha_{\min }^{n}=b^{\prime}=M_{w}^{n}\left(2 N^{n} M_{w}^{n} \eta+1\right)+$ $4 N^{n} \eta^{2}$ and $\alpha_{\max }^{n}=\frac{1}{a^{\prime}}=\frac{1}{8 N^{n} \eta^{2}}$. Thus, working with $\alpha^{n}=\alpha_{\min }^{n}$ minimizes the related dissipation. In particular, in the Low-Mach limit we have $\alpha_{\min }^{n}=4 N^{n}=4 C^{n} \frac{\Delta t c_{0}}{h}$.

Energy balance According to the previous section we have

$$
\begin{aligned}
\Delta t S_{i}^{n} & \leq w_{i}^{n} K_{1} \Delta t c_{0}^{2} \rho_{i}^{n}\left\|v_{0}^{n}\right\|_{\infty} \\
& \leq w_{i}^{n} K_{2} \Delta t \rho_{i}^{n}
\end{aligned}
$$

where $K_{2}=K_{1} c_{0}^{2}\left\|v_{0}^{n}\right\|_{\infty}$

And

$$
\begin{aligned}
Q_{i}^{n}+R_{i}^{n} \leq & \Delta t \frac{w_{i}^{n}}{w_{i}^{n+1}} q^{n}\left(\gamma^{n}\right) \sum_{j}\left[\frac{\widetilde{\kappa}_{i j}^{\prime n}}{2} c_{0}\left(\rho_{j}^{n}-\rho_{i}^{n}\right)^{2}\left\|\boldsymbol{A}_{i j}^{n}\right\| w_{j}^{n}\right] \\
& +\Delta t \frac{w_{i}^{n}}{w_{i}^{n+1}} r^{n}\left(\alpha^{n}\right) \sum_{j}\left[\frac{\rho_{j}^{n}}{2} c_{0}\left\|\boldsymbol{v}_{j}^{n}-\boldsymbol{v}_{i}^{n}\right\|^{2}\left\|\boldsymbol{A}_{i j}^{n}\right\| w_{j}^{n}\right] \\
& +\Delta t^{2}\left(K_{3}+K_{4}+K_{5}\left\|\boldsymbol{v}_{i}^{n}\right\|^{2}\right) \rho_{i}^{n}
\end{aligned}
$$

Thus in terms of energy,

$$
\begin{aligned}
\sum_{i} \omega_{i}^{n+1} E_{i}^{n+1}-\sum_{i} \omega_{i}^{n} E_{i}^{n}= & \Delta t \sum_{i} S_{i}^{n}+\sum_{i} w_{i}^{n+1}\left(Q_{i}^{n}+R_{i}^{n}\right) \\
\leq & K_{2} \Delta t \sum_{i}\left(w_{i}^{n} \rho_{i}^{n}\right) \\
& +q^{n}\left(\gamma^{n}\right) \Delta t \sum_{i}\left(w_{i}^{n} \sum_{j}\left[\frac{\widetilde{\kappa}_{i j}^{\prime n}}{2} c_{0}\left(\rho_{j}^{n}-\rho_{i}^{n}\right)^{2}\left\|\boldsymbol{A}_{i j}^{n}\right\| w_{j}^{n}\right]\right) \\
& +r^{n}\left(\alpha^{n}\right) \Delta t \sum_{i}\left(w_{i}^{n} \sum_{j}\left[\frac{\rho_{j}^{n}}{2} c_{0}\left\|\boldsymbol{v}_{j}^{n}-\boldsymbol{v}_{i}^{n}\right\|^{2}\left\|\boldsymbol{A}_{i j}^{n}\right\| w_{j}^{n}\right]\right) \\
& +\Delta t^{2}\left(\left(K_{3}+K_{4}\right) \sum_{i}\left(w_{i}^{n} \rho_{i}^{n}\right)+K_{5} \sum_{i}\left(w_{i}^{n} \rho_{i}^{n}\left\|\boldsymbol{v}_{i}^{n}\right\|^{2}\right)\right)
\end{aligned}
$$

Taking $\Delta t \frac{c_{0}}{h} \leq \min _{n}\left(N_{\gamma}^{n}, N_{\alpha}^{n}\right)$ and admissible $(\alpha, \gamma)$ we get finally

$$
\begin{aligned}
\sum_{i} \omega_{i}^{n+1} E_{i}^{n+1}-\sum_{i} \omega_{i}^{n} E_{i}^{n} \leq & K_{2} \Delta t \sum_{i}\left(w_{i}^{n} \rho_{i}^{n}\right) \\
& +\Delta t^{2}\left(\left(K_{3}+K_{4}\right) \sum_{i}\left(w_{i}^{n} \rho_{i}^{n}\right)+K_{5} \sum_{i}\left(w_{i}^{n} \rho_{i}^{n}\left\|\boldsymbol{v}_{i}^{n}\right\|^{2}\right)\right) \\
\leq & \Delta t K_{2} \sum_{i}\left(w_{i}^{n} \rho_{i}^{n}\right) \\
& +\Delta t^{2}\left(K_{6} \sum_{i}\left(w_{i}^{n} \rho_{i}^{n}\right)+K_{5} \sum_{i}\left(w_{i}^{n} \rho_{i}^{n}\left\|\boldsymbol{v}_{i}^{n}\right\|^{2}\right)\right) \\
\leq & \Delta t K_{2} \sum_{i}\left(w_{i}^{n} \rho_{i}^{n}\right) \\
& +\Delta t^{2}\left(K_{6} \sum_{i}\left(w_{i}^{n} \rho_{i}^{n}\right)+K_{5} \sum_{i}\left(w_{i}^{n} E_{i}^{n}\right)\right)
\end{aligned}
$$

where $K_{6 \eta}=K_{3 \eta}+K_{4}$.

Indeed we necessarily have $\sum_{i}\left(w_{i}^{n} \rho_{i}^{n}\left\|\boldsymbol{v}_{i}^{n}\right\|^{2}\right) \leq 2 \sum_{i}\left(w_{i}^{n} E_{i}^{n}\right)$.

We set $m_{n}=\sum_{i}\left(w_{i}^{n} \rho_{i}^{n}\right)$ the constant total mass of the system and $\mathcal{E}_{n}=\sum_{i}\left(w_{i}^{n} E_{i}^{n}\right)$ its total energy. We can write (82) as 


$$
\mathcal{E}_{n+1}-\mathcal{E}_{n} \leq \Delta t^{2} K_{5} \mathcal{E}_{n}+\Delta t m_{n} K_{2}+K_{6 \eta} \Delta t^{2} m_{n}
$$

Corresponding to

$$
\forall n, \quad \mathcal{E}_{n+1} \leq \mathcal{E}_{n}(1+\theta)+\Delta t C_{1}+\Delta t^{2} C_{2 \eta}
$$

with $\theta=\Delta t^{2} K_{5}, C_{1}=m_{n} K_{2}$ and $C_{2 \eta}=m_{n} K_{6 \eta}$.

We set $B_{\eta}=\Delta t C_{1}+\Delta t^{2} C_{2 \eta}$ giving

$$
\forall n, \quad \mathcal{E}_{n+1} \leq \mathcal{E}_{n}(1+\theta)+B_{\eta}
$$

Considering a RK2 Heun time integration scheme (27) we actually have

$$
\forall n, \quad \overline{\mathcal{E}}_{n+1} \leq \mathcal{E}_{n}(1+\theta)+B_{\eta}
$$

But thanks to the convexity of the total energy we also have

$$
\forall n, \mathcal{E}_{n+1} \leq \frac{1}{2}\left[\mathcal{E}_{n}+\overline{\mathcal{E}}_{n+1}+\theta \overline{\mathcal{E}}_{n+1}+B_{\eta}\right]
$$

Giving

$$
\forall n, \quad \mathcal{E}_{n+1} \leq \mathcal{E}_{n}(1+\xi)+D_{\eta}
$$

where $\xi=\theta\left(1+\frac{\theta}{2}\right)$ and $D_{\eta}=B_{\eta}\left(1+\frac{\theta}{2}\right)$.

Thus we know thanks to Gronwall theorem that

$$
\forall t \leq T, \mathcal{E}(t) \leq \mathcal{E}_{\text {lim }}(T)
$$

with $\mathcal{E}_{\text {lim }}(T)=(1+\xi)^{\frac{T}{\Delta t}}\left(\mathcal{E}_{0}+\frac{D_{\eta}}{\xi}\right)-\frac{D_{\eta}}{\xi}$

$$
=\mathcal{E}_{0}+C_{1} T+o(1) \text { when } \Delta t \rightarrow 0
$$

Finally, (84) insures the limited behavior of the system total energy, giving then the expected stability property of the scheme.

\subsubsection{Consistency Estimates}

Statement In addition of result (84) we can exhibit the following estimates by enforcing stricter stability conditions

$$
\exists\left(\mathcal{E}_{1}, \mathcal{E}_{2}\right) \in \mathbb{R}^{+, 2} /\left\{\begin{array}{l}
\sum_{n, i, j} w_{i}^{n} w_{j}^{n}\left(\rho_{j}^{n}-\rho_{i}^{n}\right)^{2}\left\|\boldsymbol{A}_{i j}^{n}\right\| \Delta t \leq \mathcal{E}_{1} \\
\sum_{n, i, j} w_{i}^{n} w_{j}^{n}\left\|\boldsymbol{v}_{j}^{n}-\boldsymbol{v}_{i}^{n}\right\|^{2}\left\|\boldsymbol{A}_{i j}^{n}\right\| \Delta t \leq \mathcal{E}_{2}
\end{array}\right.
$$

Proof In order to establish the stability conditions of section 9.2.4 we imposed during the estimation process a negativity condition on $q(76)$ and $r$ (77). Thus by being more restrictive (corresponding to a lower CFL condition) we can also take $\delta \in \mathbb{R}^{+, *}$ such that

$$
\left\{\begin{array}{l}
q^{n}\left(\gamma^{n}\right) \leq-\delta \\
r^{n}\left(\alpha^{n}\right) \leq-\delta
\end{array}\right.
$$

In this case according to (81) and (83) with $C_{E}^{n}=\Delta t \sum_{i, j}\left[w_{i}^{n} w_{j}^{n}\left\|\boldsymbol{A}_{i j}^{n}\right\| \frac{c_{0}}{2}\left(\widetilde{\kappa}_{i j}^{\prime n}\left(\rho_{j}^{n}-\rho_{i}^{n}\right)^{2}+\rho_{j}^{n}\left\|\boldsymbol{v}_{j}^{n}-\boldsymbol{v}_{i}^{n}\right\|^{2}\right)\right]$ we can write

$$
\overline{\mathcal{E}}_{n+1}+\delta C_{E}^{n} \leq \mathcal{E}_{n}(1+\theta)+B_{\eta}
$$

and then

$$
\mathcal{E}_{n+1}+\delta\left(1+\frac{\theta}{2}\right) C_{E}^{n} \leq \mathcal{E}_{n}(1+\xi)+D_{\eta}
$$

According to (84) we know that $\mathcal{E}_{n+1}$ and $\mathcal{E}_{n}$ are bounded insuring then the bounded behavior of $C_{E}^{n}$. Thus, both part of $C_{E}^{n}$ being positive $\exists\left(\delta_{1}^{n}, \delta_{2}^{n}\right) \in \mathbb{R}^{+, 2} /$

$$
\left\{\begin{array}{l}
\Delta t \sum_{i, j}\left[w_{i}^{n} w_{j}^{n}\left\|\boldsymbol{A}_{i j}^{n}\right\| \frac{c_{0}}{2} \widetilde{\kappa}_{i j}^{\prime n}\left(\rho_{j}^{n}-\rho_{i}^{n}\right)^{2}\right] \leq \delta_{1}^{n} \\
\Delta t \sum_{i, j}\left[w_{i}^{n} w_{j}^{n}\left\|\boldsymbol{A}_{i j}^{n}\right\| \frac{c_{0}}{2} \rho_{j}^{n}\left\|\boldsymbol{v}_{j}^{n}-\boldsymbol{v}_{i}^{n}\right\|^{2}\right] \leq \delta_{2}^{n}
\end{array}\right.
$$


Leading to

$$
\left\{\begin{array}{l}
\Delta t \sum_{i, j}\left[w_{i}^{n} w_{j}^{n}\left\|\boldsymbol{A}_{i j}^{n}\right\|\left(\rho_{j}^{n}-\rho_{i}^{n}\right)^{2}\right] \leq \frac{2 \rho_{\max }}{c_{0}^{3}} \delta_{1}^{n} \\
\Delta t \sum_{i, j}\left[w_{i}^{n} w_{j}^{n}\left\|\boldsymbol{A}_{i j}^{n}\right\|\left\|\boldsymbol{v}_{j}^{n}-\boldsymbol{v}_{i}^{n}\right\|^{2}\right] \leq \frac{2}{\rho_{\text {min }} c_{0}} \delta_{2}^{n}
\end{array}\right.
$$

Finally we get the expected consistency estimates

$$
\left\{\begin{array}{l}
\sum_{n, i, j} w_{i}^{n} w_{j}^{n}\left\|\boldsymbol{A}_{i j}^{n}\right\|\left(\rho_{j}^{n}-\rho_{i}^{n}\right)^{2} \Delta t \leq \mathcal{E}_{1} \\
\sum_{n, i, j} w_{i}^{n} w_{j}^{n}\left\|\boldsymbol{A}_{i j}^{n}\right\|\left\|\boldsymbol{v}_{j}^{n}-\boldsymbol{v}_{i}^{n}\right\|^{2} \Delta t \leq \mathcal{E}_{2}
\end{array}\right.
$$

by taking $\mathcal{E}_{1}=\sum_{n} \frac{2 \rho_{\max }}{c_{0}^{3}} \delta_{1}^{n}$ and $\mathcal{E}_{2}=\sum_{n} \frac{2}{\rho_{\text {min }} c_{0}} \delta_{2}^{n}$. 